\title{
RehabILITATION Robotics
}

STIMULATING RESTORATION OF

ARM FUNCTION AFTER STROKE

GB (Gerdienke) Prange 
Address of correspondence:

Gerdienke Prange

Roessingh Research \& Development

PO Box 310

7500 AH Enschede

the Netherlands

$+31-(0) 53-4875759$

g.prange@rrd.nl

The publication of this thesis was generously supported by:

Roessingh Research \& Development, Enschede

Roessingh Rehabilitation Center, Enschede

Chair Biomedical Signals and Systems, University of Twente, Enschede

Baat Medical, Hengelo (www.baatmedical.com)

Hocoma AG, Volketswil, Switzerland (www.hocoma.ch/en)

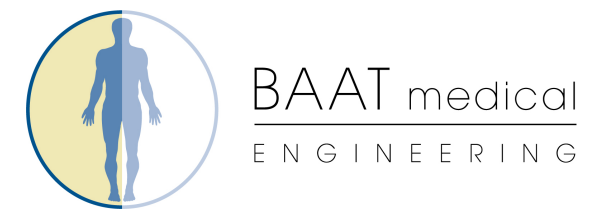

\section{Ill Hocoma}

Printed by Gildeprint Drukkerijen, Enschede, the Netherlands

Cover design: Jurriaan van Hengel

ISBN 978-90-365-2901-3

(C) Gerdienke Prange, Enschede, the Netherlands, 2009

All rights reserved. No part of this book may be reproduced, stored in a retrieval system, or transmitted, in any form or by any means, electronic, mechanical, photocopying, recording, or otherwise, without prior written permission of the holder of the copyright. 


\section{REHABILITATION ROBOTICS}

STIMULATING RESTORATION OF ARM FUNCTION AFTER STROKE

\section{ProefsChRIfT}

ter verkrijging van

de graad van doctor aan de Universiteit Twente, op gezag van de rector magnificus,

prof. dr. H. Brinksma, volgens besluit van het College voor Promoties in het openbaar te verdedigen op donderdag 15 oktober 2009 om 15.00 uur

door

Grada Berendina Prange

geboren op 18 juni 1981

te Aalten 
Dit proefschrift is goedgekeurd door de promotoren en assistent-promotor:

Prof. dr. ir. HJ Hermens

Prof. dr. MJ IJzerman

Dr. MJA Jannink 
De promotiecommissie is als volgt samengesteld:

Voorzitter:

Prof. dr. ir. AJ Mouthaan Universiteit Twente

Promotor:

Prof. dr. ir. $\mathrm{HJ}$ Hermens Universiteit Twente

Co-promotor:

Prof. dr. MJ IJzerman

Universiteit Twente

Assistent-promotor:

Dr. MJA Jannink

Universiteit Twente

Leden:

Prof. dr. ir. PH Veltink

Universiteit Twente

Prof. dr. ir. HFJM Koopman

Universiteit Twente

Prof. dr. JS Rietman

Universiteit Twente

Prof. dr. JH Burridge

University of Southampton, United Kingdom

Deskundige:

Dr. P Schenk

Hocoma AG, Switzerland

Paranimfen:

Drs. Karlijn Cranen

Dr. Laura Kallenberg 



\section{Table of Contents}

$\begin{array}{lll}\text { Chapter } 1 \text { General Introduction } & 9\end{array}$

Chapter 2 Systematic review of the effect of robot-aided therapy on 23 recovery of the hemiparetic arm after stroke

Chapter 3 Influence of gravity compensation on muscle activity during reach and retrieval in healthy elderly

Chapter 4 Influence of gravity compensation on muscle activation patterns during different temporal phases of arm movements of stroke patients

Chapter 5 An explorative, cross-sectional study into abnormal muscle 89 synergies during functional reach in chronic stroke patients

Chapter 6 Changes in muscle activation after reach training with gravity compensation in chronic stroke patients

Chapter 7 General Discussion

Summary

Samenvatting

Dankwoord

Over de auteur

Publications 



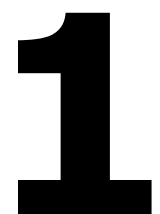

General InTRODUCTION 



\section{General Introduction}

\section{Stroke consequences}

A cerebrovascular accident, or stroke, is a major cause of mortality or permanent disability. The annual incidence rate of stroke in the Netherlands is approximately 250 per $100,000 .{ }^{1}$ The rate in the US is comparable with a yearly incidence of approximately 260 stroke patients per 100,000 inhabitants. $^{2}$ The percentage of people suffering a stroke increases strongly with age. ${ }^{2}$

Stroke can be defined as a neurological deficit due to damage to the blood supply of the brain, either ischemic (i.e., obstruction of a blood vessel; occurring in approximately $85 \%$ ) or hemorrhagic (i.e., rupture of a blood vessel; occurring in approximately $15 \%){ }^{2}$ A stroke causes a destruction of brain tissue in areas that are subjected to blood deprivation. This can result in a variety of sensory, motor, cognitive and psychological symptoms, such as sensory loss, hemispatial neglect, aphasia, muscle weakness, spasticity, limited movement coordination, attention and memory deficits, depression and behavioral changes. ${ }^{3}$ Concerning the motor domain, a stroke leads to damage of nerve pathways between the brain and the spinal cord and to reduced integration of sensory and motor information during motor planning in the brain. Such impaired conduction of nerve signals from motor areas of the cortex to the spinal cord limits selective activation of muscle tissue. With respect to the upper extremity, impaired arm and hand function may cause serious limitations in activities of daily living for the majority of stroke patients. Directly after stroke, upper extremity weakness is the most common impairment, occurring in $77 \%$ of patients with a first-ever stroke. ${ }^{4}$ Longitudinal follow-up studies revealed that $60 \%$ of stroke patients regain very little dexterity after 6 months. ${ }^{5}$

After stroke, spontaneous neurological recovery of motor function occurs, but the extent varies largely between persons. Regaining functional use of the affected arm is typically limited to a group of stroke survivors that experiences some recovery of function of the lower extremity at 1 week after stroke and/or of the arm after 4 weeks post-stroke. ${ }^{5}$ From a clinical perspective, spontaneous recovery has been described to follow a relatively stereotypical sequence, which can take several weeks up to several months and can halt at any stage. ${ }^{6,7}$ After an initial stage of paresis, muscle tone increases and reflexes become hyperactive. Next, some voluntary movement returns, but this is restricted to rather stereotypical patterns of movement. Subsequently, more selective voluntary movement 
becomes possible and a normalization of muscle tone is observed. Finally, completely voluntary movements return, without any restriction to stereotypical movement patterns. ${ }^{6}$ Such stereotypical movement patterns can be observed as involuntary coupling of movements over multiple joints, generally involving two types: a flexion synergy and an extension synergy. ${ }^{7}$ For the upper extremity, the flexion synergy pattern consists of shoulder abduction, shoulder external rotation, elbow flexion and forearm supination, while the extension synergy pattern comprises shoulder adduction, shoulder internal rotation, elbow extension and forearm pronation. In general, the flexion synergy develops prior to the extension synergy in the arm, and recovery mostly progresses from proximal to distal, although exceptions may be observed. ${ }^{6}$

This process of spontaneous neurological recovery can involve several short-term and long-term physiological mechanisms in the brain. One of the mechanisms underlying neurological recovery after stroke is enhancement of active brain tissue surrounding the actual damaged area. Shortly after the stroke, edema in the tissue surrounding the infarct is reduced (both intracellular and extracellular), the ischemic penumbra is resolved (i.e., reperfusion of the blood deprived brain area) and diaschisis (i.e., malfunction of remote brain areas due to lack of neural input) is diminished, so that related brain areas can regain their neural communication. ${ }^{3,8,9}$

A longer-term mechanism involved in neurological recovery is neural plasticity, meaning that brain activity and cortical representations of motor actions change during recovery. ${ }^{3,8,9}$ This cortical reorganization can occur in areas adjacent to and remote from the infarcted area. Processes involved in cortical reorganization can include activation of previously inactive neurons (i.e., unmasking of latent synapses), facilitation of alternative networks, and collateral sprouting (i.e., growth of new axons). ${ }^{3,9}$ These processes allow for the development of new paths for neural communication, to circumvent those that were damaged by the stroke. ${ }^{8}$

\section{Stroke rehabilitation}

One of the aims of stroke rehabilitation is to stimulate restoration of arm function. More than half a century ago, rehabilitation of upper extremity dysfunction after stroke was regarded as an orthopedic problem, managed by bracing, surgery and muscle re-education (i.e., training of individual muscles). ${ }^{10}$ However, this approach had limited effectiveness in treating abnormal movement patterns. ${ }^{11}$

Gradually, attention shifted towards neurofacilitation techniques. ${ }^{10,11}$ Neurofacilitation techniques involve the notion that abnormal patterns are 
unwanted and need to be suppressed or controlled and that normal movement can be facilitated by sensory input. ${ }^{12}$ An example is the Bobath approach, whose basic principles are used in conventional rehabilitation by a large part of the physical therapists in the Netherlands. ${ }^{13}$ However, it has been recognized that such treatment approaches may not be optimal to stimulate restoration of arm function. ${ }^{14}$ Research revealed no difference in efficacy between various neurofacilitation techniques or between those methods and more conventional approaches teaching re-education or compensational strategies. ${ }^{12,15-17}$

Focus shifted once again, towards an approach based on the system's theory, in which integration of multiple components is thought to result in organized, normal movement. ${ }^{10,11}$ In rehabilitation, this translates to goal-directed exercises, where multi-joint movements come together in one meaningful activity resembling activities of daily living, instead of non-representative single-joint movements. Nowadays, stroke rehabilitation generally includes aspects of different approaches, ranging from muscle re-education to neurofacilitation techniques and repetitive task practice. ${ }^{12}$ None of these approaches have shown to be superior to another in the treatment of motor dysfunction of the upper extremity in patients suffering from stroke. ${ }^{18}$ Therefore, emphasis has been placed more and more on evidencebased physical therapy during the last decades, leading to increasing research into the effectiveness of therapeutic interventions.

Currently, several effective physical therapeutic interventions for treatment of the arm after stroke have been identified, ${ }^{16-18}$ and accumulated into recommendations for (Dutch) stroke rehabilitation. ${ }^{13}$ For the upper extremity, constrained-induced movement therapy, consisting of forced-use of the affected arm and hand in combination with extensive functional training, is one of the approaches that has shown positive effects on arm and hand function in mildly affected stroke patients. ${ }^{10,19}$ Neuromuscular electrical stimulation of the wrist and finger extensors may also induce improvements in arm and hand function. ${ }^{20,21}$ However, these beneficial changes are limited to stroke patients having at least some wrist and finger extension ability.

Besides this research into effectiveness of interventions, more and more studies investigated underlying mechanisms of motor recovery. Principles of motor relearning and processes of cortical reorganization have provided a neurophysiological basis for key aspects that have the potential for stimulation of restoration of arm function after stroke. ${ }^{9,22}$ These key aspects, which should be applied in exercise therapy for optimal results, include active initiation and execution of movements, high training intensity and application of functional 
exercises.

Concerning active initiation and execution of movements, brain studies have shown that cortical activity is larger during active execution of movements than during passive motion, predominantly in secondary motor area's and basal ganglia. ${ }^{23}$ Also, motor cortex excitability is higher after active movement training, accompanied by increased agonist activity and decreased antagonist activity, in contrast to passive movement training. ${ }^{24}$ Exercise therapy focusing on active initiation and execution of movements is associated with improved arm function. ${ }^{25-}$ 27 With respect to training intensity, repetition of movements has shown to strengthen the representation of the trained movements in the brain. ${ }^{28}$ Training with a higher frequency or longer duration stimulates functional recovery of the arm. ${ }^{16,17,29-31}$ In regard to functional exercises, several studies have shown that functional training, focusing on activities of daily life that are relevant to the patient (i.e., task-specificity), results in a normalization of brain activity. ${ }^{22,32}$ Such normalization of brain activity is related to improvements in motor control and functional abilities. ${ }^{33}$ Therefore, task-specificity also is an important feature of exercise therapy to stimulate motor recovery after stroke. ${ }^{28}$

\section{Rehabilitation robotics}

Technological innovations provide an opportunity to design interventions that take many key aspects for stimulation of motor relearning into account. A promising application is the use of rehabilitation robotics to complement conventional therapy. Robotic devices have the possibility to guide movements in a very accurate and reproducible way during specific parts of a movement and through specific types of guidance, which is hard to accomplish by manual interaction between therapist and patient. The use of rehabilitation robotics is not only applicable to patients with fairly good residual arm function, but is also suitable for more severely affected patients. ${ }^{34}$ In addition to these advantages concerning treatment, the use of robotic devices offers the possibility to quantify each patient's specific impairments and his/her progress during treatment. This can be done by using sensitive and objective measures of movement performance, such as speed and smoothness of the movement and exerted forces in desired or undesired directions. ${ }^{35}$

Robotic devices can be programmed to apply forces in a smart way to guide a person's movement. This makes it possible to stimulate or facilitate desired movements that a stroke patient may not be able to make on his/her own. These forces can be applied to the hand of the person (i.e., end-effector, see figure 1.1), 
or to each joint axis separately via a frame attached to the arm (i.e., exoskeleton, see figure 1.2). Robotic devices can manipulate movements of a person in several ways. ${ }^{36}$ In passive mode, the robotic device imposes movements by moving the arm of the patient in a pre-planned trajectory, while the patient remains relaxed. In active-assisted mode, the robotic device provides assistance during active movements of the patient, when the patient is not able to complete the movement. In active-resisted mode, the robotic device delivers resistance against movements actively executed by the patient.

Several robotic devices have been developed and evaluated specifically for application in stroke rehabilitation since the 1990's. The MIT-Manus (figure 1.3), developed by the Massachusetts Institute of Technology was the first device to be evaluated extensively in clinical trials, to examine whether robot-aided therapy was an acceptable way of exercise therapy for stroke patients and if it could improve arm function of stroke patients. ${ }^{37}$ These studies were paralleled by clinical studies applying other robotic devices, such as the MIME (mirror-image motion enabler; figure 1.4), ${ }^{38}$ the ArmGuide (figure 1.5), ${ }^{39}$ and the Bi-Manu-Track (figure 1.6). ${ }^{40}$ These devices incorporate most of the above-mentioned operational modes. The MIME can additionally apply a bi-manual mode, in which movement of the lessaffected arm serves as a template for passive movement of the affected arm.

\section{Gravity compensation}

Most robotic devices incorporate another type of assistance in their design besides passive, active-assisted and active-resisted operational modes: arm support, or gravity compensation. ${ }^{41}$ The majority of devices are designed in such a way that the weight of the arm is counterbalanced, either passively by the mechanical construction or actively by applying compensating robotic forces. This basic feature

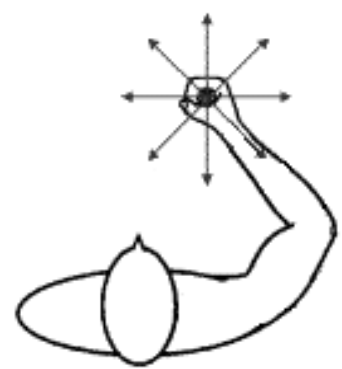

Figure 1.1 End-effector design for robot

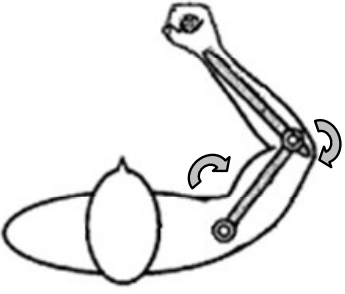

Figure 1.2 Exoskeleton design for robot 
is commonly not regarded as a separate operational mode of the device, and is therefore not controlled as a part of the exercise protocol. However, research has indicated that the sole application of arm support may influence control of arm movements. After stroke, an especially strong coupling was found between shoulder abduction and elbow flexion. ${ }^{42,43}$ By supporting the arm, the amount of shoulder abduction torques that a stroke patient has to generate to execute a movement (e.g., lifting the arm during reach) is reduced, which leads to a diminished strength of simultaneous, involuntary elbow flexion. ${ }^{44-47}$ As is suggested for the gravity compensation feature of robotic devices, each of the other operational modes (or therapy modalities) possibly influences motor control of arm movements in its own way. In order to identify the most optimal set of therapy modalities that should be incorporated in rehabilitation robotics, information about the influence of each separate modality on restoration of arm function is important.

Different mechanisms of recovery can be involved in achieving the ultimate goal of stroke rehabilitation, either conventional or robot-aided, which is functional independence of the stroke patient. Improvements in functional use of the arm in daily life can, on the one hand, be accomplished via restoration of degraded neural function, by stimulating cortical reorganization processes via the key aspects of active, intensive and functional training (i.e., restitution of function). ${ }^{8}$ On the other hand, behavioral adaptations and compensatory strategies can be used to circumvent lost motor function (i.e., substitution of function). ${ }^{8}$ For example, if a

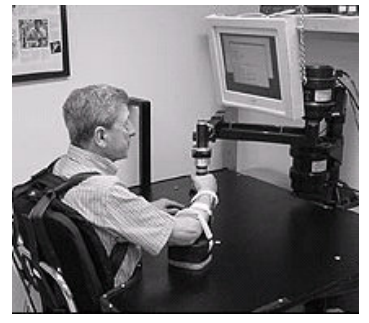

Figure 1.3 MIT-Manus

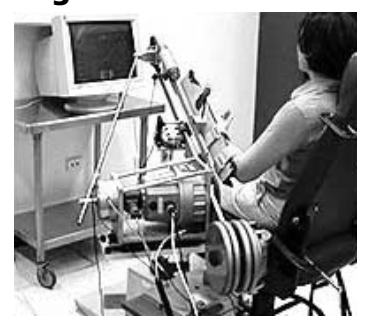

Figure 1.5 ArmGuide

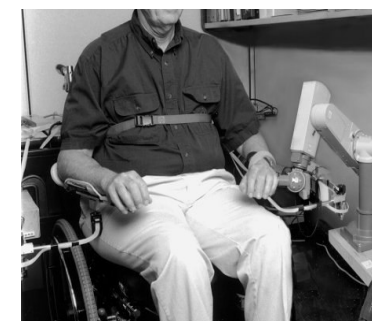

Figure 1.4 MIME

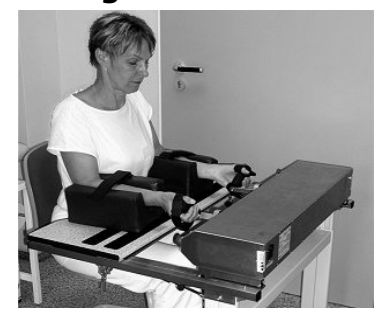

Figure 1.6 Bi-Manu-Track 
stroke patient wants to regain his ability to reach for a cup on the table, he can try to increase his ability to extend the elbow, or he can learn to bend his trunk forward instead of extending his arm. ${ }^{46}$

Many approaches intervene at the motor control level, which may change muscle activation. By investigating changes in muscle activation due to application of robotic devices, the merits of different approaches may be discerned. Information about the neuromuscular basis of changes in motor control due to application of robotic devices can also aid in understanding which mechanisms of recovery are targeted by the application of rehabilitation robotics. This knowledge can then be used to identify effective applications of robotic devices in stroke rehabilitation and how to take advantage of the merits of rehabilitation robotics, in order to design the content and timing of rehabilitation as to achieve optimal results. ${ }^{8}$

\section{Thesis outline}

The main objective of the research reported in this $\mathrm{PhD}$ thesis is to obtain a better understanding of the impact of different therapy modalities of rehabilitation robotics on neuromuscular control of arm movements of stroke patients. Gravity compensation can be considered one of the basic therapy modalities incorporated in a robotic device, and since this has not been applied or controlled as a separate modality, this was chosen as the initial focus within this $\mathrm{PhD}$ research.

In chapter 2, a systematic review is described that evaluates the effect of existing clinical studies into robot-aided rehabilitation and tries to identify therapy modalities that have the potential to enhance restoration of arm function after stroke. The findings indicate that the application of rehabilitation robotics can improve motor control of arm movements. However, the individual contribution of each operational mode could not be discerned, highlighting the need for research into the influence of separate therapy modalities on arm movements.

The way gravity compensation influences motor control of functional arm movements is largely unknown, even in healthy persons. In chapter 3, a reference frame for the neuromuscular basis of the influence of gravity compensation on functional arm movements is provided by examining this influence in healthy elderly. To apply gravity compensation, a device is designed within the scope of this research project: Freebal (figure 1.7). ${ }^{49}$

In chapter 4 , the influence of the application of gravity compensation on the ability of stroke patients to control functional arm movements and its neuromuscular basis are examined. Again, the Freebal device is used to provide gravity compensation. 
The experiments in chapters 3 and 4 give rise to a more fundamental question about the neuromuscular basis of the influence of gravity compensation, specifically concerning the role of abnormal synergies during functional arm movements. In chapter 5, the influence of abnormal coupling on motor control after stroke is investigated with a specific focus on functional arm movements. To this end, abnormal synergies are provoked by providing specific resistance during functional movements by means of another device that was designed within this research project: Dampace (figure 1.8)..$^{50}$

Combining the findings from the cross-sectional studies, it is suggested that gravity compensation has the potential to improve functional arm movements. To investigate if the instantaneous influence of gravity compensation translates to improved unsupported arm movements after a longer-term application and to examine the underlying mechanisms, chapter 6 describes a longitudinal study implementing gravity compensation as intervention during training of functional arm movements.

In the concluding chapter 7, the implications of the research reported in this PhD thesis are discussed in the context of current and promising new physical therapy interventions, and by highlighting future possibilities and technological innovations that may stimulate stroke rehabilitation even more.

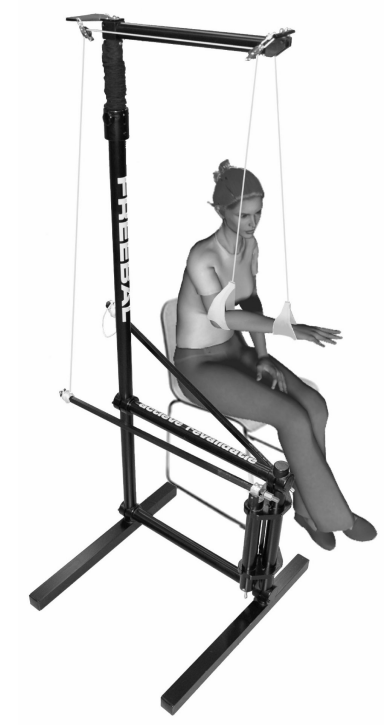

Figure 1.7 Freebal

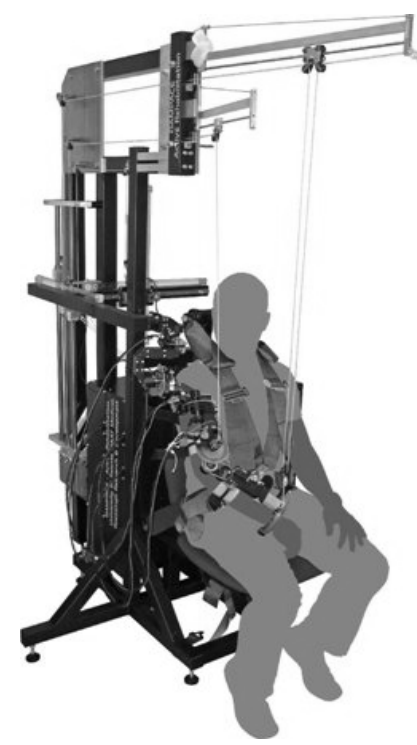

Figure 1.8 Dampace 


\section{References}

1. Bots $M L$, van Dis SJ. Factsheet Stroke (in Dutch: Cijfers en feiten - Beroerte). publication of the Dutch Heart Foundation (in Dutch: Nederlandse Hartstichting) 2006; http://www.hartstichting.nl/Uploads/Brochures/Factsheet\%20Beroerte.pdf

2. Rosamond W, Flegal K, Furie K, et al. Heart disease and stroke statistics 2008 update. Circulation 2008;117:e25-e146

3. Speach DP, Dombovy ML. Recovery from stroke: rehabilitation. Baillieres Clin Neurol $1995 ; 4(2): 317-338$

4. Lawrence ES, Coshall C, Dundas R, et al. Estimates of the prevalence of acute stroke impairments and disability in a multiethnic population. Stroke 2001;32:1279-1284

5. Kwakkel G, Kollen BJ, Van der Grond J, Prevo AJH. Probability of regaining dexterity in the flaccid upper limb: impact of severity of paresis and time since onset in acute stroke. Stroke $2003 ; 34: 2181-2186$

6. Twitchell TE. The restoration of motor function following hemiplegia in man. Brain $1951 ; 74(4): 443-480$

7. Brunnstrom S. Movement therapy in hemiplegia, a neurophysiological approach. New York: Harper \& Row, Publishers; 1970

8. Kwakkel G, Kollen B, Lindeman E. Understanding the pattern of functional recovery after stroke: Facts and theories. Restor Neurol Neurosci 2004;22:281-299

9. Krakauer JW. Arm function after stroke: from physiology to recovery. Semin Neurol 2005;25(4):384-395

10. Wolf SL, Blanton S, Baer H, Breshears J, Butler AJ. Repetitive task practice: A critical review of constraint-induced movement therapy in stroke. Neurol 2002;8:325-338

11. Mathiowetz V, Bass Haugen J. Motor behavior research: implications for therapeutic approaches to central nervous system dysfunction. Am J Occup Ther 1994; 48(8):733-745

12. Zorowitz RD, Gross E, Polinski DM. The stroke survivor. Disabil Rehabil 2002; 24(13):666-679

13. Van Peppen RPS, Kwakkel G, Harmeling-van der Wel BC, et al. Guideline for stroke by KNGF [in Dutch: KNGF-richtlijn beroerte). Ned Tijdschr Fysiother 2004;114 (5;supplement)

14. Mayston M. Editorial: Bobath concept: Bobath@50: mid-life crisis - What of the future? Physiother Res Int 2008;13(3):131-136

15. Dickstein R, Hocherman S, Pillar T, Shaham R. Stroke Rehabilitation - Three exercise therapy approaches. Phys Ther 1986;66(8):1233-1238

16. Wagenaar RC, Meijer OG. Effects of stroke rehabilitation: a critical review of the literature. J Rehabil Sci 1991;4(3):61-73

17. Platz T. Evidence-based rehabilitation of the arm. A systematic review [Evidenzbasierte Armrehabilitation. Eine systematische Literaturübersicht]. 
Nervenarzt 2003;74:841-849

18. Van der Lee JH, Snels IA, Beckerman H, Lankhorst GJ, Wagenaar RC, Bouter LM. Exercise therapy for arm function in stroke patients: a systematic review of randomized controlled trials. Clin Rehabil 2001;15:20-31

19. Dromerick AW , Edwards DF, Hahn M. Does the application of constrained-induced movement therapy during acute rehabilitation reduce arm impairment after ischemic stroke? Stroke 2000;31:2984-2988

20. De Kroon JR, Van der Lee JH, IJzerman MJ, Lankhorst GJ. Therapeutic electrical stimulation to improve motor control and functional abilities of the upper extremity after stroke: a systematic review. Clin Rehab 2002;16(4):350-360

21. IJzerman MJ, Renzenbrink GJ, Geurts ACH. Neuromuscular stimulation after stroke: from technology to clinical deployment. Expert Rev Neurother 2009;9(4):541-552

22. Schaechter JD. Motor rehabilitation and brain plasticity after hemiparetic stroke. Prog Neurobiol 2004;73:61-72

23. Weiller $C$, Jüptner $M$, Fellows $S$, et al. Brain representation of active and passive movements. Neuroimage 1996;4:105-110

24. Kaelin-Lang A, Sawaki L, Cohen LG. Role of voluntary drive in encoding an elementary motor memory. J Neurophysiol 2005;93:1099-1103

25. Feys HM, De Weerdt WJ, Selz BE, et al. Effect of a therapeutic intervention for the hemiplegic upper limb in the acute phase after stroke. A single-blind, randomized, controlled multicenter trial. Stroke 1998;29:785-792

26. Barreca S, Wolf SL, Fasoli S, Bohannon R. Treatment interventions for the paretic upper limb of stroke survivors: a critical review. Neurorehabil Neural Repair 2003; 17:220-226

27. Kahn LE, Lum PS, Rymer WZ, Reinkensmeyer DJ. Robot-assisted movement training for the stroke-impaired arm: Does it matter what the robot does? J Rehabil Res Dev 2006;43(5):619-630

28. Fisher BE, Sullivan KJ. Activity-dependent factors affecting poststroke functional outcomes. Top Stroke Rehabil 2001;8(3):31-44

29. Kwakkel G, Wagenaar RC, Twisk JWR, Lankhorst GJ, Koetsier JC. Intensity of leg and arm training after primary middle-cerebral-artery stroke: a randomised trial. Lancet 1999;354:189-194

30. Kwakkel G, Wagenaar RC, Koelman TW, Lankhorst GJ, Koetsier JC. Effects of intensity of rehabilitation after stroke. A research synthesis. Stroke 1997;28(8):15501556

31. Kwakkel G, Van Peppen R, Wagenaar RC, et al. Effects of augmented exercise therapy time after stroke: a meta-analysis. Stroke 2004;35(11):2529-2539

32. Nelles G, Jentzen W, Jueptner M, Müller S, Diener HC. Arm training induced brain plasticity in stroke studied with serial positron emission tomography. Neuro Image 2001;13:1146-1154

33. Feydy A, Carlier R, Roby-Brami A, et al. Longitudinal study of motor recovery after 
stroke: recruitment and focusing of brain activation. Stroke 2002;33:1610-1617

34. Fasoli SE, Krebs HI, Hogan N. Robotic technology and stroke rehabilitation: translating research into practice. Top Stroke Rehabil 2004; 11(4):11-19

35. Krebs HI, Volpe BT, Ferraro $M$, et al. Robot-aided neurorehabilitation: from evidence-based to science-based rehabilitation. Top Stroke Rehabil 2002;8(4):54-70

36. Lum P, Reinkensmeyer D, Mahoney R, Rymer WZ, Burgar C. Robotic devices for movement therapy after stroke: current status and challenges to clinical acceptance. Top Stroke Rehabil 2002;8(4):40-53

37. Krebs HI, Hogan N, Aisen ML, Volpe BT. Robot-aided neurorehabilitation. IEEE Trans Rehabil Eng 1998;6(1):75-87

38. Burgar CG, Lum PS, Shor PC, Machiel Van der Loos HF. Development of robots for rehabilitation therapy: the Palo Alto VA/Stanford experience. J Rehabil Res Dev 2000; 37(6):663-673

39. Reinkensmeyer DJ, Kahn LE, Averbuch M, McKenna-Cole A, Schmit BD, Rymer WZ. Understanding and treating arm movement impairment after chronic brain injury: progress with the ARM guide. J Rehabil Res Dev 2000;37(6):653-662

40. Hesse S, Schulte-Tigges G, Konrad M, Bardeleben A, Werner C. Robot-assisted arm trainer for the passive and active practice of bilateral forearm and wrist movements in hemiparetic subjects. Arch Phys Med Rehabil 2003; 84(6):915-920

41. Johnson MJ. Recent trends in robot-assisted therapy environments to improve reallife functional performance after stroke. J Neuroengineering Rehabil 2006;3:29

42. Dewald JPA, Pope PS, Given JD, Buchanan TS, Rymer WZ. Abnormal muscle coactivation patterns during isometric torque generation at the elbow and shoulder in hemiparetic subjects. Brain 1995;118:495-510

43. Beer RF, Given JD, Dewald JPA. Task-dependent weakness at the elbow in patients with hemiparesis. Arch Phys Med Rehabil 1999;80:766-772

44. Beer RF, Dewald JPA, Rymer WZ. Deficits in the coordinaton of multijoint arm movements in patients with hemiparesis: evidence for disturbed control of limb dynamics. Exp Brain Res 2000;131:305-319

45. Dewald JPA, Sheshadri V, Dawson ML, Beer RF. Upper-limb discoordination in hemiparetic stroke: implications for neurorehabilitation. Top Stroke Rehabil 2001;8(1):1-12

46. Beer RF, Dewald JPA, Dawson ML, Rymer WZ. Target-dependent differences between free and constrained arm movements in chronic hemiparesis. Exp Brain Res 2004; 156:458-470

47. Beer RF, Ellis MD, Holubar BG, Dewald JPA. Impact of gravity loading on post-stroke reaching and its relationship to weakness. Muscle Nerve 2007;36:242-250

48. Cirstea MC, Levin MF. Compensatory strategies for reaching in stroke. Brain 2000;123:940-953

49. Stienen AHA, Hekman EEG, Van der Helm FCT, Prange GB, Jannink MJA, Aalsma $A M M$, and Van der Kooij $H$. Freebal: dedicated gravity compensation for het upper 
extremities. In: Proceedings of the 10th International Conference on Rehabilitation Robotics (ICORR); June 13-15, 2007, Noordwijk aan Zee, the Netherlands:804-808

50. Stienen AHA, Hekman EEG, Van der Helm FCT, Prange GB, Jannink MJA, Aalsma AMM, and Van der Kooij H. Dampace: dynamic force-coordination trainer for the upper extremities. In: Proceedings of the 10th International Conference on Rehabilitation Robotics (ICORR); June 13-15, 2007, Noordwijk aan Zee, the Netherlands:820-826 
SYSTEMATIC REVIEW OF THE EFFECT OF ROBOT-AIDED THERAPY ON RECOVERY OF THE HEMIPARETIC ARM AFTER STROKE

GB Prange

MJA Jannink

CGM Groothuis-Oudshoorn

HJ Hermens

MJ IJzerman

J Rehabil Res Dev 2006;43(2):171-184 


\section{Abstract}

A limited number of clinical studies have examined the effect of post-stroke rehabilitation with robotic devices on hemiparetic arm function. We systematically reviewed the literature to assess the effect of robot-aided therapy on stroke patients' upper-limb motor control and functional abilities. Eight clinical trials were identified and reviewed. For four of these studies, we also pooled short-term mean changes in Fugl-Meyer scores before and after robot-aided therapy. We found that robot-aided therapy of the proximal upper limb improves short- and long-term motor control of the paretic shoulder and elbow in sub-acute and chronic patients; however, we found no consistent influence on functional abilities. In addition, robot-aided therapy appears to improve motor control more than conventional therapy. 


\section{Introduction}

A cerebrovascular accident (CVA), or stroke, is a sudden ischemic or hemorrhagic disturbance in the blood supply to brain tissue that results in partial loss of brain function. The incidence of stroke in the Netherlands is 162 per 100,000 people, which means approximately 25,000 new patients each year. ${ }^{1}$ In the United States, approximately 500,000 people $(171$ per 100,000$)$ experience a stroke each year. ${ }^{2}$ This high stroke incidence, in combination with an aging population, which implies future increases in incidence, greatly strains national healthcare services and related costs.

A stroke causes partial destruction of cortical tissue and results in disturbed generation and integration of neural commands. The interrupted generation and integration of neural commands from the sensorimotor areas of the cortex results in a reduced or even absent ability to selectively activate muscle tissue, which affects motor task performance. A consequence of disturbed neural command generation in the sensorimotor cortex is impaired arm and hand motor function. ${ }^{3}$ Optimal restoration of arm and hand motor function is essential for stroke patients to independently perform activities of daily living (ADL).

High-intensity and task-specific upper-limb treatment consisting of active, highly repetitive movements is one of the most effective approaches to arm and hand function restoration. ${ }^{4-6}$ Unfortunately, standard multidisciplinary stroke rehabilitation is labor-intensive and requires one-to-one manual interactions with therapists. Treatment protocols entail daily therapy for several weeks, which makes the provision of highly intensive treatment for all patients difficult. ${ }^{7}$ In addition, the evaluation of patients' performance and progress is usually subjective because few adequate objective measures are available. ${ }^{7,8}$

Given these problems in stroke rehabilitation, researchers saw an opportunity to create new, technological solutions. The use of robotic devices in rehabilitation can provide high-intensity, repetitive, task-specific, and interactive treatment of the impaired upper limb and an objective, reliable means of monitoring patient progress. With robotic devices, patients may achieve increased gains from rehabilitation treatment.

Many research groups have developed robotic devices for upper-limb rehabilitation, for example, Massachusetts Institute of Technology (MIT)-Manus, ${ }^{9}$ Assisted Rehabilitation and Measurement (ARM) Guide, ${ }^{10}$ Mirror Image Motion Enabler (MIME), ${ }^{11}$ Bi-Manu-Track, ${ }^{12}$ GENTLE/S, ${ }^{13}$ Neurorehabilitation Robot 
(NeReBot) ${ }_{1}^{14}$ REHAROB,${ }^{15}$ Arm Coordination Training 3-D (ACT3D) ${ }^{16}$ and ARMin. ${ }^{17}$ The training these devices provide is based on exercise therapy modalities that the literature and/or clinical practice indicate may help restore upper-limb motor control and function. One such modality is passive movement, in which the robotic device moves the patient's arm (possible in all robotic devices). Another modality is active movement that is either partially assisted by the robotic device, in the case of some voluntary but inadequate function (possible with all robotic devices), or resisted by the robotic device, in the case of voluntary and selective function (only evaluated in MIT-Manus, Bi-Manu-Track, MIME). ${ }^{9,11,12} \mathrm{~A}$ further modality is bimanual exercise, in which active movement of the unaffected arm is mirrored by simultaneous passive movement of the affected arm by the robotic device (only possible in Bi-Manu-Track and MIME). ${ }^{11,12}$ In most robotic systems, more than one modality is incorporated into a single robotic device. Most robotic devices were designed for training the proximal upper limb (shoulder and elbow) of the hemiparetic arm by enabling movement in multiple directions. ${ }^{9-11,13,14,16,18,19}$ The $\mathrm{Bi}$ Manu-Track focuses on the distal upper $\operatorname{limb}$ (forearm and wrist), ${ }_{1}^{12}$ as does a recent extension of the MIT-Manus robotic device for training of wrist movements. ${ }^{20}$ New robotic devices and evolutions of existing devices are continuously being designed (e.g., Furusho et al. ${ }^{21}$ and Colombo et al. $^{22}$ ) and include several systems for training hand movements (e.g., the force feedback glove of Merians et al. ${ }^{23}$ and the devices of Kline et al. ${ }^{24}$ and Mulas et al. ${ }^{25}$ ).

The design and development of robotic devices have been reported extensively, but only a few clinical studies, which varied in design and methods, have examined the effect of robotic devices on stroke rehabilitation in a clinical setting.

Insight into the use of robot-aided therapy can be obtained through systematic analysis of the literature. Our main objective in performing this systematic analysis was to investigate the effect of robot-aided therapy on the upper-limb motor control and functional abilities of stroke patients. 


\section{Methods}

\section{Literature search}

We conducted a systematic search of articles from 1975 to August 2005 in the PubMed, Cochrane Controlled Trials (Rehabilitation and Related Therapies), Center for International Rehabilitation Research Information and Exchange (CIRRIE, http://cirrie.buffalo.edu), and National Rehabilitation Information Center for Independence REHABDATA (http://www.naric.com) databases. CIRRIE includes research from all areas of rehabilitation conducted outside the United States from 1990 to 2005. We consulted REHABDATA for rehabilitation research conducted within the United States.

We used the following key words in these searches: arm, arms, cerebrovascular accident, CVA, hemiplegia, hemipleg*, hemiparesis, hemipare*, robotics, robot*, stroke, upper extremities, upper extremity, upper limb, and upper limbs. The search strategy that we used for PubMed is presented in Appendix 2.1 This strategy was adjusted to suit the other databases. In addition to searching the databases, we checked the references of relevant publications and scanned the proceedings of the 2005 Institute of Electrical and Electronics Engineers $9^{\text {th }}$ International Conference on Rehabilitation Robotics (Chicago, Illinois) for the most up-to-date developments in rehabilitation robotics.

\section{Study selection}

Two reviewers independently selected and summarized studies and scored their methodological quality. The reviewers met regularly to discuss their findings and decisions. In the case of disagreement, a third reviewer was consulted.

To be selected for review, a study had to: (1) be a clinical trial (i.e., compare pre- and post-treatment performance) or controlled trial (i.e., clinical trial with a control group, either randomized or not); (2) Involve stroke patients; (3) concern movement therapy with a robotic device; (4) focus on upper-limb motor control (and possibly functional abilities); (5) use relevant motor control and functional ability outcome measures; (6) be a full-length publication in a peer-reviewed journal. Studies on the application of robotic devices for purposes other than therapeutic treatment (e.g., studies on ADL support aids) were excluded. To enable the most complete view of the current literature, we did not limit the search by patient subgroups (i.e., acute, sub-acute, or chronic) or by language.

In this review, we use "motor control" to indicate aspects of impairments in body 
functions and upper-limb structures (e.g., decreased strength) and "functional abilities" to indicate limitations in activities (e.g., inability to reach an object).

\section{Methodological quality judgment}

We selected studies with a variety of designs rather than only randomized controlled trials (RCTs), although RCTs provide the most reliable data on intervention effectiveness. We decided this because this research area is relatively young and only a few clinical studies on upper-limb robot-aided therapy after stroke have been published. The standard items for scoring the methodological quality of RCTs are not suitable for other study designs. Therefore, to evaluate the methodological quality of the selected studies, we applied Kottink et al.' $\mathrm{s}^{26}$ adapted list of methodological items based on the Maastricht-Amsterdam criteria for RCTs. ${ }^{27}$ This list contains 16 items on patient selection, intervention, outcome measurement, and statistics, each of which was scored as positive (yes), negative (no), or unclear (don't know). Each positive score received 1 point and each negative or unclear score received 0 points, with the exception of the study design item, which varied from 1 point for uncontrolled studies to 3 points for RCTs (RCT designs are less sensitive to bias). Thus, the maximum methodological quality score was 19.

\section{Data extraction}

We analyzed the contents of the selected studies using a structured diagram. By filling in this diagram, we were able to scan the general contents of the studies for: (1) descriptive features of the subjects; (2) intervention(s) implemented in the study; (3) outcome measures for evaluation of the effects on both motor control and functional abilities; (4) conclusions based on the results. The extracted conclusions were considered positive if the change between pre- and posttreatment measurements or the difference between robot-trained and control groups was significantly different $(a<0.05)$ as calculated by a statistical test appropriate to the research question and the data characteristics.

\section{Data analysis}

In addition to the qualitative interpretation of studies, we performed a quantitative analysis for more objective insight into the effect of robot-aided therapy on motor control recovery. The primary outcome measure for quantification of motor recovery was the upper-limb portion of the Fugl-Meyer (FM) assessment. Using a data-pooling model appropriate to the characteristics and data of the selected 
studies, we pooled short-term changes in FM score before and immediately after robot-aided therapy into a mean difference across studies and calculated the 95 percent confidence interval (CI) of this pooled FM difference. 


\section{Results}

\section{Study selection}

From the systematic literature search, we identified 17 clinical trials. Of these, 11 were clinical studies from the group that implemented the MIT-Manus beginning in 1997. These publications included several consecutive clinical trials and summaries of those clinical trials and often used the same subjects. ${ }^{9,28-33}$ Of these 11 studies, only the most representative summary of the clinical trials was included in our analysis ${ }^{32}$ along with four separate articles that were clearly dissimilar in research question or experimental setup from the studies in the summary. ${ }^{34-37}$ Three studies that used the MIME also met the selection criteria. The second and third MIME studies $^{38,39}$ used the same subjects as the first MIME study, ${ }^{11}$ so we excluded the first study. Although the second and third studies used the same subjects, we included both because they focused on two separate aspects of robot-aided therapy (biomechanics ${ }^{38}$ and muscle activation patterns ${ }^{39}$ ).

All selected studies concentrated on the restoration of proximal upper-limb function by training of the shoulder and elbow, except for two studies that tested a robotic device (Bi-Manu-Track) for training of the forearm and wrist. ${ }^{12,40}$ Since distal upper-limb training is a different application than proximal upper-limb training, synthesis of the research would have been problematic. Therefore, these two Bi-Manu-Track studies were excluded. This reduced the number of selected studies to the eight studies summarized in table $2.1 \mathrm{a}$ and $2.1 \mathrm{~b} . .^{10,32,34-39}$

During data extraction, the two raters disagreed on 6 of the 80 general content items (8\%). These disagreements were resolved through discussion and the third reviewer was not consulted.

\section{Methodological quality judgment}

Two of the selected studies were experimental trials with pre- and post-treatment measurements of both an experimental and control group, ${ }^{32,38}$ of which one was an RCT. ${ }^{38}$ The remaining six studies had a pre- and post-treatment measurement design for robot-aided therapy without a control group. 10,34-37,39

The wide range of study designs included in our review was reflected in the methodological quality scores that ranged from $8^{10}$ to $16^{36,38}$ out of a possible 19 points. The two raters disagreed on 7 of the 128 methodological quality items (5\%). Again, these disagreements were resolved through discussion and the third reviewer was not consulted. 
Table 2.1a Study characteristics and results

\begin{tabular}{|c|c|c|c|c|c|c|c|c|}
\hline Author (year) & \multicolumn{2}{|l|}{ Krebs $2000^{32}$} & \multicolumn{2}{|l|}{ Ferraro $2003^{34}$} & \multicolumn{2}{|l|}{ Fasoli 2004 ${ }^{35}$} & \multicolumn{2}{|l|}{ Stein $2004^{36}$} \\
\hline Robotics & \multicolumn{2}{|c|}{$\begin{array}{l}\text { MIT-Manus; passive, active- } \\
\text { assisted + -resisted movement }\end{array}$} & \multicolumn{2}{|c|}{$\begin{array}{l}\text { MIT-Manus; passive, active- } \\
\text { assisted + -resisted movement }\end{array}$} & \multicolumn{2}{|c|}{$\begin{array}{l}\text { InMotion2 (previous MIT-Manus); } \\
\text { active-assisted + -resisted }\end{array}$} & \multicolumn{2}{|c|}{$\begin{array}{l}\text { InMotion2 (previous MIT-Manus); } \\
\text { active-assisted OR -resisted }\end{array}$} \\
\hline Patients & \multicolumn{2}{|l|}{$\begin{array}{l}E: n=40 \\
C: n=36\end{array}$} & \multicolumn{2}{|l|}{$E: n=30$} & \multicolumn{2}{|l|}{$E: n=42$} & \multicolumn{2}{|l|}{$E: n=46^{+}$} \\
\hline Diagnosis & \multicolumn{2}{|c|}{$\begin{array}{l}\text { E: sub-acute stroke } \\
\text { C: sub-acute stroke }\end{array}$} & \multicolumn{2}{|c|}{ E: chronic stroke } & \multicolumn{2}{|c|}{ E: chronic stroke } & \multicolumn{2}{|c|}{ E: chronic stroke } \\
\hline $\begin{array}{l}\text { Age } \\
(\text { mean } \pm S D)\end{array}$ & \multicolumn{2}{|c|}{$\begin{array}{l}\mathrm{E}: 61.1 \pm 4.4 \mathrm{yr}^{*} \\
\text { C: } 65.9 \pm 5.7 \mathrm{yr}^{*}\end{array}$} & \multicolumn{2}{|l|}{$\mathrm{E}: 64.8 \pm 2.3 \mathrm{yr}$} & \multicolumn{2}{|l|}{ E: $57.4 \pm 13.9 \mathrm{yr}$} & \multicolumn{2}{|c|}{ E: $57.6 \pm 13.6 \mathrm{yr}$} \\
\hline $\begin{array}{l}\text { Time post-stroke } \\
(m e a n \pm S D)\end{array}$ & \multicolumn{2}{|c|}{$\begin{array}{l}\text { E: } 2.2 \pm 0.3 \mathrm{wk}^{*} \\
\text { C: } 2.6 \pm 0.7 \mathrm{wk}^{*}\end{array}$} & \multicolumn{2}{|c|}{ E: $1299 \pm 147 \mathrm{~d}(\approx 43 \mathrm{mo})$} & \multicolumn{2}{|c|}{ E: $28.7 \pm 12.4 \mathrm{mo}$} & \multicolumn{2}{|c|}{ E: $26.1 \pm 12.4 \mathrm{mo}$} \\
\hline Intervention & \multicolumn{2}{|c|}{$\begin{array}{l}\text { E: conventional therapy + robot } \\
\text { training; } 4-5 \mathrm{~h} / \mathrm{wk} \text { for } 6 \mathrm{wk} \\
\text { C: conventional therapy }+ \\
\text { exposure to robot; } 1 \mathrm{~h} / \mathrm{wk} \text { for } 6 \mathrm{wk}\end{array}$} & \multicolumn{2}{|c|}{ E: robot training; $3 \mathrm{~h} / \mathrm{wk}$ for $6 \mathrm{wk}$} & \multicolumn{2}{|c|}{ E: robot training; $3 \mathrm{~h} / \mathrm{wk}$ for $6 \mathrm{wk}$} & \multicolumn{2}{|c|}{$\begin{array}{l}\text { E: robot training; } 3 \mathrm{~h} / \mathrm{wk} \text { for } 6 \mathrm{wk} \\
\text { (divided in two groups; receiving } \\
\text { either assisted or resisted robot } \\
\text { training) }\end{array}$} \\
\hline Follow-up & \multicolumn{2}{|l|}{3 yr $(n=6)$} & \multicolumn{2}{|l|}{$3 \mathrm{mo}$} & \multicolumn{2}{|l|}{$4 \mathrm{mo}(\mathrm{n}=40)$} & \multicolumn{2}{|l|}{-} \\
\hline \multirow[t]{2}{*}{ Outcome } & motor control & funct. ability & motor control & funct. ability & motor control & funct. ability & motor control & funct. ability \\
\hline & $\begin{array}{l}\text { FM } \\
\text { MSS (s/e) } \\
\text { MSS (w/h) } \\
\text { MP } \\
\text { kinematics } \\
\text { (indiv.data) }\end{array}$ & & $\begin{array}{l}\text { FM } \\
\text { MSS(s/e) } \\
\text { MSS(w/h) } \\
\text { MP } \\
\text { AS }\end{array}$ & & $\begin{array}{l}\text { FM } \\
\text { MSS (s/e) } \\
\text { MSS (w/h) } \\
\text { MP } \\
\text { AS } \\
\text { MVC }\end{array}$ & FIM & $\begin{array}{l}\text { FM } \\
\text { MSS (s/e) } \\
\text { MSS (w/h) } \\
\text { MP } \\
\text { AS } \\
\text { pain }\end{array}$ & \\
\hline Conclusions & \multicolumn{2}{|c|}{$\begin{array}{l}\text { Robot training improved all } \\
\text { parameters. Improvement was } \\
\text { larger for MSS(s/e) and MP } \\
\text { compared to control group. } \\
\text { Long term sustenance only for } \\
\text { MSS(s/e). }\end{array}$} & \multicolumn{2}{|c|}{$\begin{array}{l}\text { Robot training improved FM and } \\
\text { MP (also sustained at follow-up). } \\
\text { Improvements favored moderate } \\
\text { stroke pt. Improvement in FIM } \\
\text { found for moderate severity stroke } \\
\text { group only. }\end{array}$} & \multicolumn{2}{|c|}{$\begin{array}{l}\text { Robot training improved FM, MSS } \\
\text { (s//) and MP, also maintained on } \\
\text { follow-up. Also reduction in } \\
\text { shoulder pain both short and long } \\
\text { term. } \\
\text { Effects were training specific! }\end{array}$} & \multicolumn{2}{|c|}{$\begin{array}{l}\text { After robot training FM, MSS (s/e) } \\
\text { and MVC improved significantly. } \\
\text { NB: No significant difference } \\
\text { between assisted and resisted } \\
\text { robot training! }\end{array}$} \\
\hline Meth. quality & \multicolumn{2}{|l|}{12} & \multicolumn{2}{|l|}{14} & 15 & & 16 & \\
\hline
\end{tabular}

Table 2.1b continued

\begin{tabular}{|c|c|c|c|c|c|c|c|c|}
\hline Author (year) & \multicolumn{2}{|l|}{ Krebs $2004^{37}$} & \multicolumn{2}{|l|}{ Lum 2002 38} & \multicolumn{2}{|l|}{ Lum 2004 } & \multicolumn{2}{|c|}{ Reinkensmeyer $2000^{10}$} \\
\hline Robotics & \multicolumn{2}{|c|}{$\begin{array}{l}\text { MIT-Manus; passive, active- } \\
\text { assisted +-resisted movement }\end{array}$} & \multicolumn{2}{|c|}{$\begin{array}{l}\text { MIME; passive, active-assisted + } \\
\text { active-resisted movement }\end{array}$} & \multicolumn{2}{|c|}{$\begin{array}{l}\text { MIME; passive, active-assisted + } \\
\text { resisted movement }\end{array}$} & \multicolumn{2}{|c|}{$\begin{array}{l}\text { ARM Guide; passive + active- } \\
\text { assisted movement }\end{array}$} \\
\hline Patients & \multicolumn{2}{|l|}{$E: n=9$} & \multicolumn{2}{|l|}{$\begin{array}{l}E: n=13 \\
C: n=14\end{array}$} & \multicolumn{2}{|l|}{$E: n=13$} & \multicolumn{2}{|l|}{$E: n=3$} \\
\hline Diagnosis & \multicolumn{2}{|c|}{ E: chronic stroke } & \multicolumn{2}{|c|}{$\begin{array}{l}\text { E: chronic stroke } \\
\text { C: chronic stroke }\end{array}$} & \multicolumn{2}{|c|}{ E: chronic stroke } & \multicolumn{2}{|c|}{ E: chronic stroke } \\
\hline $\begin{array}{l}\text { Age (year) } \\
\text { (mean } \pm S D)\end{array}$ & \multicolumn{2}{|l|}{$\mathrm{E}: 62.0 \pm 4.3 \mathrm{yr}$} & \multicolumn{2}{|l|}{$\begin{array}{l}\mathrm{E}: 63.2 \pm 3.6 \mathrm{yr} \\
\text { C: } 65.9 \pm 2.4 \mathrm{yr}\end{array}$} & \multicolumn{2}{|l|}{$\mathrm{E}: 63.2 \pm 12.8 \mathrm{yr}$} & \multicolumn{2}{|l|}{$\mathrm{E}: 41 \pm 9.6 \mathrm{yr}$} \\
\hline $\begin{array}{l}\text { Time post-stroke } \\
\text { (mean } \pm S D)\end{array}$ & \multicolumn{2}{|c|}{$\mathrm{E}: 50.0 \pm 8.9 \mathrm{mo}$} & \multicolumn{2}{|c|}{$\begin{array}{l}\mathrm{E}: 30.2 \pm 6.2 \mathrm{mo} \\
\mathrm{C}: 28.8 \pm 6.3 \mathrm{mo}\end{array}$} & \multicolumn{2}{|c|}{ E: $30.2 \pm 22.2 \mathrm{mo}$} & \multicolumn{2}{|l|}{$\mathrm{E}: 4.3 \pm 1.7 \mathrm{yr}$} \\
\hline Intervention & \multicolumn{2}{|c|}{$\begin{array}{l}\text { E: robot training in horizontal plane; } \\
3 \mathrm{~h} / \mathrm{wk} \text { for } 6 \mathrm{wk} \\
+ \text { in robot training in vertical plane; } \\
3 \mathrm{~h} / \mathrm{wk} \text { for } 6 \mathrm{wk}\end{array}$} & \multicolumn{2}{|c|}{$\begin{array}{l}\text { E: robot training; } 3 \mathrm{~h} / \mathrm{wk} \text { for } 8 \mathrm{wk} \\
\text { C: conventional therapy (NDT); } \\
3 \mathrm{~h} / \mathrm{wk} \text { for } 8 \mathrm{wk}+\text { non-contact } \\
\text { exposure to robot }\end{array}$} & \multicolumn{2}{|c|}{ E: robot training; $3 \mathrm{~h} / \mathrm{wk}$ for $8 \mathrm{wk}$} & \multicolumn{2}{|c|}{$\begin{array}{l}\text { E: robot training; } 3 \mathrm{~h} / \mathrm{wk} \text { for } 8 \mathrm{wk} \\
(\mathrm{n}=2) \text { or } 4 \mathrm{wk}(\mathrm{n}=1)\end{array}$} \\
\hline Follow-up & \multicolumn{2}{|l|}{-} & \multicolumn{2}{|l|}{$6 \mathrm{mo}$} & \multicolumn{2}{|l|}{-} & \multicolumn{2}{|l|}{-} \\
\hline \multirow[t]{2}{*}{ Outcome } & motor control & funct. ability & motor control & funct. ability & motor control & funct. ability & motor control & funct. ability \\
\hline & $\begin{array}{l}\text { FM } \\
\text { MSS (s/e) } \\
\text { MP } \\
\text { AS }\end{array}$ & & $\begin{array}{l}\text { FM } \\
\text { MVC } \\
\text { kinematics }\end{array}$ & $\begin{array}{l}\text { BI } \\
\text { FIM }\end{array}$ & $\begin{array}{l}\text { EMG } \\
\text { strength } \\
\text { active ROM } \\
\text { kinematics }\end{array}$ & & $\begin{array}{l}\text { active ROM } \\
\text { tone } \\
\text { kinetics + } \\
\text { kinematics }\end{array}$ & \\
\hline Conclusions & \multicolumn{2}{|c|}{$\begin{array}{l}\text { Reduction in impairment after robot } \\
\text { training in vertical plane was not } \\
\text { significant for shoulder/elbow parts } \\
\text { of FM, MSS, MP and AS, but } \\
\text { comparable to i iprovement during } \\
\text { planar robot training. No } \\
\text { information about wrist/hand. }\end{array}$} & \multicolumn{2}{|c|}{$\begin{array}{l}\text { Robot group improved in all motor } \\
\text { control measures (short- and long- } \\
\text { term), but only long-term for both } \\
\text { functional abilities measures. } \\
\text { Robot group improved more than } \\
\text { control in motor control (only short- } \\
\text { term) and in functional abilities } \\
\text { (only long-term) in all parameters. }\end{array}$} & \multicolumn{2}{|c|}{$\begin{array}{l}\text { Significant improvement after robot } \\
\text { training in kinematics and EMG } \\
\text { (predominantly for reach at } \\
\text { shoulder level and not at elbow } \\
\text { height). }\end{array}$} & \multicolumn{2}{|c|}{$\begin{array}{l}\text { Robot training improved AROM, } \\
\text { peak movement speed and tone; } \\
\text { results of incoordination and free } \\
\text { reaching inconsistent. }\end{array}$} \\
\hline Meth. quality & \multicolumn{2}{|l|}{11} & \multicolumn{2}{|l|}{16} & 10 & & 8 & \\
\hline
\end{tabular}

Abbreviations: FM = Fugl-Meyer assessment; MSS = Motor Status Scale; MP = Motor Power scale; AS = modified Ashworth Scale; ROM = range of motion; MVC = maximal voluntary contraction; FIM = Functional Independence Measure; BI = Barthel Index; * Data from two separate but related studies since this information was not in current article; we averaged mean and pooled variance over all participants; +18 subjects had very severe impairments and could not participate in randomization; thus $n=28$ subjects were randomized across groups and analyzed 


\section{Patients}

The size of the experimental groups ranged from $3^{10}$ to $42^{35}$ (total $n=178$ ) and the sizes of the two control groups were $14^{38}$ and $36^{32}$ (total $n=50$ ). Except for the studies with $3^{10}$ and $9^{37}$ patients in the experimental group, all other studies involved at least 13 patients. Krebs et al.'s summary of multiple trials involved patients with sub-acute stroke (defined as 2 to 4 weeks post-stroke, $n=40$ in the experimental groups and $n=36$ in the control groups). ${ }^{32}$ The remaining seven studies involved chronic stroke patients (defined as $>6$ months post-stroke, $n=138$ in the experimental groups and $n=14$ in the control groups). ${ }^{10,34-39}$

\section{Intervention}

Three different robotic devices were used for intervention across the eight studies: the MIT-Manus system was used in five studies, ${ }^{32,34-37}$ the MIME in two studies, ${ }^{38,39}$ and the ARM Guide in one study. ${ }^{10}$ Technical details of these systems can be found elsewhere. ${ }^{10,11,32}$ All eight studies included short-term pre- and post-treatment measurements, but only four studies included long-term assessments (varying from 3 mo to 3 yr post-treatment) of the influence of robot-aided therapy. ${ }^{32,34,35,38}$

The intervention in each study was robot-aided therapy. The training sessions were similar in seven studies and consisted of repetitive, goal-directed forwardreaching movements (i.e., the upper limb had to be stretched forward to reach the goal and the movement was not necessarily confined to two dimensions). The movements were actively performed by the subject (possibly partially assisted by the robotic device). ${ }^{10,32,34-36,38,39}$ One exception was the pilot evaluation of training of vertical (upward) movements that were assisted by MIT-Manus. ${ }^{37}$ In Krebs et al.'s summary study, ${ }^{32}$ robot-aided therapy supplemented the conventional rehabilitation program. The other studies only used robot-aided therapy.

Participants in control groups received conventional therapy with additional noncontact or nonoperational exposure to the robot (i.e., the robot was turned off). The nature of the conventional therapy was not stated explicitly in those studies. ${ }^{32,38}$

\section{Outcome measures}

A total of 12 separate outcome measures were used across all studies: 10 motor control measures and 2 functional ability measures. Each study measured at least four different outcomes. All eight studies assessed motor control and six used the upper-limb portion of the FM. ${ }^{32,34-38}$ Only two studies measured functional abilities; both used the Functional Independence Measure (FIM). ${ }^{34,38}$ 


\section{Content of studies}

Seven studies reported short-term improvements in motor control as a result of forward-directed robot-aided therapy (table 1). The one study that investigated vertical movements did not report a significant improvement in motor control, although the trend was positive. ${ }^{37}$ All four studies that assessed the long-term effects of robot-aided therapy on motor control reported significant improvements. $^{32,34,35,38}$ Of the two studies that measured functional abilities, one found significant short-term improvements after robot-aided therapy ${ }^{34}$ and the other reported long-term progress. ${ }^{38}$

The two studies that compared data from a robot-trained group with a control group reported significant differences in predominantly short-term measures of motor control. ${ }^{32,38}$ These differences favored robot-aided therapy. No such trend was reported for functional abilities.

All studies reported that the effects of robot-aided therapy were training-specific: improvements occurred only in the trained shoulder and elbow, and no increase in motor control or functional abilities occurred in the wrist or hand. None of the studies reported adverse effects of robot-aided therapy.

\section{Data analysis}

Five of the eight studies measured pre- and post-treatment upper-limb FM scores after forward-directed robot-aided therapy. Of these five studies, one studied subacute $^{32}$ and four studied chronic $\mathrm{c}^{34-36,38}$ stroke patients. We performed a quantitative meta-analysis of the four studies that involved chronic stroke patients. ${ }^{34-36,38}$ Data presentation was insufficient in two of these studies ${ }^{34,38}$ because the standard deviation (SD) of the change in FM scores was missing; this left two studies ( $n=70$ in total in the experimental groups) for estimation of a pooled mean difference in FM scores before and after robot-aided therapy. ${ }^{35,36} \mathrm{~A}$ standard chi-square test for heterogeneity ${ }^{41}$ showed non-significant systematic variation between these two studies $(x 2=0.683, p=0.43)$; therefore, we used the fixed-effect model to estimate the pooled mean difference in FM scores. ${ }^{42}$

Change in FM score (i.e., difference between average pre- and post-treatment scores, including SD) and corresponding 95 percent CI for each study are presented in table 2.2 and displayed graphically in figure 2.1. A summary of the results of the two studies with sufficient data showed that robot-aided therapy positively influenced FM scores: the pooled average FM score increased 3.7 points $(95 \%-\mathrm{CI}=2.8-4.7)$. This indicates a statistically significant $6 \%$ increase in motor control after robot-aided therapy $(p<0.05)$. The methodological quality scores of all 
studies are also included in table 2.2 and figure 2.1. Methodological quality and reported changes in FM scores did not appear to be related.

To include estimated results of the two studies with missing SD, we performed a sensitivity analysis. ${ }^{43}$ We calculated the adjusted pooled mean difference and the corresponding 95 percent CI for several possible SD values (ranging from 1 to 10 for both incomplete studies). This analysis showed that the adjusted pooled mean difference could vary from 4.0 to 4.7 points and the corresponding 95 percent CI from 3.2 to 6.2 points. Thus, the influence of robot-aided therapy is still positive when these two studies are incorporated. Results of the meta-analysis of the four studies support findings the of qualitative analysis of the eight selected studies.

Table 2.2 Change in Fugl-Meyer scores after robot-aided therapy in chronic patients

\begin{tabular}{|c|c|c|c|c|}
\hline Study & $\begin{array}{l}\text { Methodological } \\
\text { quality }\end{array}$ & $\mathbf{N}$ & $\begin{array}{l}\text { Change in FM score } \\
\text { (mean } \pm \text { SD) }\end{array}$ & 95\%-CI \\
\hline Ferraro 2003*,34 & 14 & 28 & 6.1 (no SD) & - \\
\hline Fasoli $2004^{35}$ & 15 & 42 & $3.4 \pm 4.0$ & 2.2 to 4.6 \\
\hline Stein $2004+{ }^{36}$ & 16 & 28 & $4.2 \pm 4.2$ & 2.6 to 5.8 \\
\hline Lum 2002*, 38 & 16 & 13 & 4.7 (no SD) & - \\
\hline Pooled average§ & & 70 & $3.7 \pm 0.5$ & 2.8 to 4.7 \\
\hline \multicolumn{5}{|c|}{$\begin{array}{l}\text { Abbreviations: } \mathrm{N}=\text { number of subjects; FM = Fugl-Meyer assessment; SD = standard deviation; CI = } \\
\text { confidence interval } \\
\text { * cumulative mean changes of shoulder/elbow and wrist/hand components of FM assessment; no SD } \\
\text { due to presentation of before and after treatment scores, so difference in SD's could not be calculated } \\
{ }^{+} \text {data pooling of } 4 \text { groups with two separate robot training regimens (mean: average of } 4 \text { groups; } \\
\text { standard deviation: based on pooled estimation of variance of } 4 \text { groups) } \\
{ }^{*} \text { data derived from graphical display of results } \\
\S \text { pooled average difference in FM and total number of subjects based on two studies with sufficient } \\
\text { data (i.e. including SD of change in FM score) }\end{array}$} \\
\hline
\end{tabular}




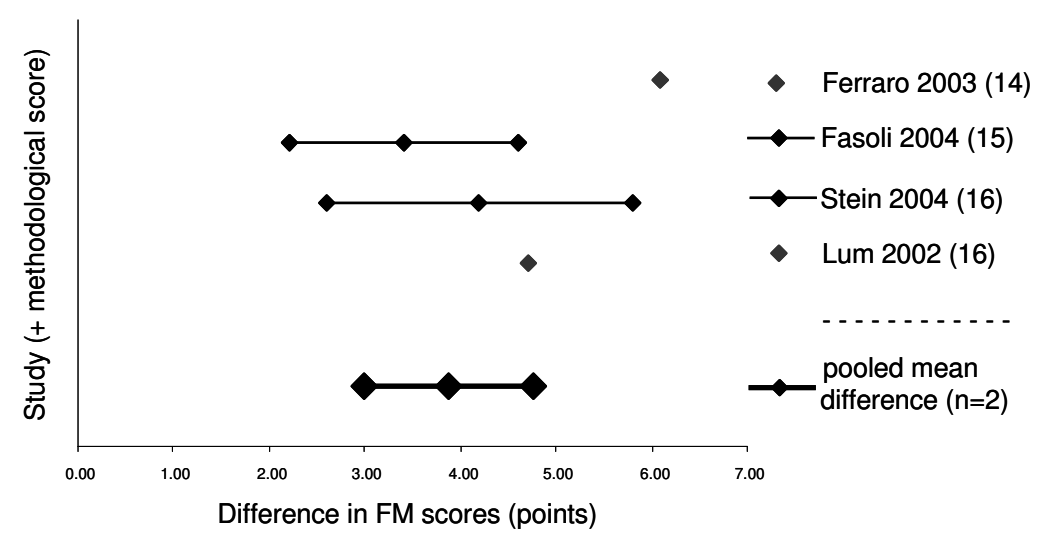

Figure 2.1 Change in Fugl-Meyer scores after robot-aided therapy in chronic patients

Notes: Mean differences pre- and post-treatment (middle symbol on line), with corresponding 95\%-CI (outer symbols on line), are displayed for each individual study. The methodological quality score of each study is included between brackets. Pooled mean difference in Fugl-Meyer score (middle symbol on bold line) is presented with the corresponding pooled $95 \%$-CI (outer symbols on bold line), based on the two studies with sufficient data. 


\section{Discussion}

In this systematic review, we qualitatively analyzed eight studies to assess the effect of robot-aided therapy on improvement of upper-limb motor control and functional abilities in stroke patients. The results of the analysis show that forwarddirected robot-aided therapy improves several motor-control aspects (e.g., muscle activation patterns, selectivity, and speed of movement) and has long-term effects of several months to several years, as measured at follow-up. The additional quantitative data analysis of short-term changes in upper-limb FM scores supported the positive influence of robot-aided therapy on motor recovery in chronic stroke patients (the pooled mean FM scores after robot-aided therapy increased by $6 \%$ ). Reinkensmeyer et al. presented comparable findings in a narrative overview of published research, ${ }^{44}$ including some earlier MIT-Manus studies. In contrast, the Krebs et al. study that assessed vertical-movement training showed no significant change in motor control, although the results showed a distinct positive trend. ${ }^{37}$ No consistent influence of robot-aided therapy on improvement of functional abilities could be detected from the qualitative analysis. This inconclusive finding is consistent with the minimal effect of interventions such as Bobath, neurodevelopmental therapy, proprioceptive neuromuscular facilitation, and Brunnstrom on ADL performance after stroke rehabilitation. ${ }^{45}$

In studies with a robot-trained group and a control group, robot-aided therapy caused more short-term reduction in motor impairments, such as muscle activation patterns and selectivity and speed of movement, than conventional rehabilitation techniques. For functional abilities, no difference was found between the robottrained and control groups. Only one study examined long-term effects so no firm conclusion can be drawn.

Research in this field is relatively young and few large RCTs have been conducted. Therefore, the factors that might affect the outcome of robot-aided therapy and bias current research findings are still unclear.

An obvious factor affecting the conclusions is that we included studies with both sub-acute and chronic stroke patients. No apparent difference in the positive influence of robot-aided therapy was found between these patient groups when we looked at all outcome measures qualitatively, but the number of included studies (one sub-acute, seven chronic) is too small for us to draw firm conclusions. Nevertheless, this information indicates that both sub-acute and chronic stroke 
patients can improve upper-limb function after robot-aided therapy. Although this result seems to contradict the traditional assumption that most recovery occurs within the first 3 to 6 months after stroke with no further improvements later on ${ }^{46,47}$ it is consistent with several more recent publications that claim that chronic patients (i.e., more than 6 months post-stroke) can improve upper-limb function. ${ }^{1,48,49}$ The pooled mean increase in chronic stroke patients' FM scores after robot-aided therapy support the results of these recent studies.

A second potential bias is the difference in treatment intensity between experimental and control groups. In Krebs et al.'s summary study, ${ }^{32}$ the robottrained group received more hours of therapy (robot-aided therapy plus conventional therapy) than the control group (conventional therapy alone). This difference may have overestimated the effect of robot-aided therapy, since higher intensity therapy has been reported to lead to better results than lower intensity therapy. ${ }^{6,50,51}$ Nonetheless, the potential motor-control benefit of robot-aided therapy over conventional therapy is supported by the positive findings of the other controlled trial, in which the robot-trained and control groups received equal treatment intensity. ${ }^{38}$

A third possible bias is the use of the upper-limb portion of FM for quantitative analysis. Although the influence of robot-aided therapy was training-specific in all eight studies (i.e., only detectable in shoulder and elbow), only the score of the upper-limb portion of the FM was reported; therefore, this measure (which included assessment of wrist and hand recovery) was used for pooling data. Calculation of total scores for assessment of training-specific improvements in motor control might have underestimated the influence of robot-aided therapy on clinical outcome. This may explain, in part, why the 6 percent increase in the upper-limb FM score after robot-aided therapy was statistically significant but not clinically relevant ( $\geq 10 \%$ increase in FM score $)^{52}$. Thus, robot-aided therapy may be even more beneficial for reduction of motor impairments than postulated by our results.

Despite these potential limitations, our results justify more in-depth study of the positive effects of robot-aided therapy and the factors that influence these effects.

One interesting aspect concerns the different response to robot-aided therapy by different patient groups. Ferraro et al. distinguished between moderately and severely affected stroke patients (based on Canadian Neurological Scale and National Institutes of Health Stroke Scale stroke-severity estimates) and reported that moderately affected patients were more responsive to robot-aided therapy than severely affected patients. ${ }^{34}$ This finding is consistent with other authors' 
findings that high initial motor function likely promotes positive strokerehabilitation outcome in general. ${ }^{1,46,47,53}$ Another study in our review reported similar results: the patients with the highest initial motor function increased more on motor-control measures after robot-aided therapy than the patients with the lowest initial motor function. ${ }^{36}$ Accordingly, inclusion of subgroup analyses based on stroke severity in future studies would more extensively assess motor-outcome differences after robot-aided therapy and expand our knowledge of the patient groups that benefit most from robot-aided therapy.

A second interesting aspect of our review concerns training specificity. In the studies we analyzed, only the shoulder and elbow were trained and only motor control of the shoulder and elbow improved. However, proper wrist and hand use is particularly relevant for functional use of the hemiparetic arm. The focus on the shoulder and elbow in these studies may partially explain the lack of functional ability improvement after robot-aided therapy. So, future attention on the wrist and hand may maximize the gains from robot-aided therapy. Research groups have recognized the importance of studying the wrist and hand and are extending their robot devices with distal trainers. ${ }^{54}$ One group developed a separate robot device for distal upper-limb training and performed two clinical studies with encouraging results on enhanced recovery of pronation-supination and wrist flexion-extension with the Bi-Manu-Track device. ${ }^{12,40}$

A third interesting but unclear aspect is the role of individual treatment modalities in robot-aided therapy. Most trials implemented three different modalities in one robotic treatment protocol: passive, active-assisted, and activeresisted movements. However, these modalities were generally not tested separately; only the overall effect of robot-aided therapy was evaluated. One treatment modality may be much more effective than another. Only Fasoli et al. ${ }^{33}$ and Stein et al. ${ }^{36}$ tried to specify the separate contributions of treatment modalities. Fasoli et al.'s results suggest that robot-aided therapy that incorporates active-resisted movements is more beneficial than active-assisted therapy for upper-limb recovery because a carryover effect on motor control in the untrained wrist and hand was found only after active-resisted therapy with the trained proximal arm. ${ }^{33}$ However, in a replication study by the same research group, a larger sample of participants revealed no differences in motor recovery between active-assisted and active-resisted therapy, possibly because of differences in analytic methods. ${ }^{36}$ The Fasoli et al. study included patients who did not have sufficient initial function to participate in the active-resisted training group and were "allocated" to the robot-assisted group instead, ${ }^{33}$ which was not the case in 
Stein et al. ${ }^{36}$ Consequently, results from the active-resisted training group were overestimated. These findings show that certain types of robot-aided therapy may be less appropriate for certain patient groups. However, the contribution of individual training modalities to restoration of upper-limb function remains ambiguous.

Other treatment modalities may be relevant and suitable for incorporation into robot-aided therapy but have not yet been extensively explored. One possible modality is compensation of gravity for the upper limb. Most robotic devices provide some arm support, a feature that Sanchez et al. outlined..$^{55}$ However, Beer et al. specifically researched the potential of compensation of gravity on the upper limb. ${ }^{56-59}$ In previous research, they found that several muscle activation patterns are coupled in abnormal synergies during active torque generation in the hemiparetic upper limb (e.g., shoulder abduction by stroke patients impaired their ability to extend the elbow). ${ }^{60}$ In subsequent research, they demonstrated that stroke patients' motor performance improved instantaneously without the influence of gravity on the upper limb because of a diminished need for active shoulder abduction and consequently increased ability to extend the elbow in static conditions. ${ }^{56,57}$ Recent preliminary results suggest that the same mechanisms apply to dynamic conditions. ${ }^{58,59}$ These findings indicate that compensation of gravity may stimulate improved motor performance of the upper limb. However, in a trial of single-case series, a group that received training with sling suspension of the upper limb (to support the arm against gravity) showed no improvement with respect to baseline. ${ }^{13}$ Compensation of gravity, while fairly unexplored, may further stimulate upper-limb recovery in stroke patients. More research on this topic may give insight into which mechanisms are influenced by gravity compensation and may identify potential applications.

In addition to robot-aided therapy, other treatment types may effectively stimulate recovery after stroke. For example, preliminary results of Kahn et al.'s pilot study suggested that a group that received robot-assisted reach training (target is always reached) and a group that attempted to reach independently (no obligation to reach the target) had similar improvements in motor control. ${ }^{61}$ Other approaches to stroke rehabilitation that have shown promising results include functional electrical stimulation, ${ }^{62}$ pharmacology, ${ }^{63}$ and several exercise therapy approaches, $^{50}$ including repetitive exercise ${ }^{61}$ constraint induced therapy, ${ }^{64}$ including a recently developed automated application; ${ }^{65,66}$ and sensorimotor training. ${ }^{5}$ However, overall comparisons of the effectiveness of several treatments are not abundant, and the available overviews are not conclusive as to the best 
approach for assisting recovery after stroke. ${ }^{4,67,68}$ Future research should shed more light on the effectiveness of these interventions compared with or even in combination with one another.

\section{Conclusions}

This systematic review indicates that robot-aided therapy of the proximal upper limb can improve short- and long-term motor control of the paretic shoulder and elbow. This conclusion is supported by a quantitative analysis of short-term pooled FM data in chronic stroke patients and indicates that increased motor recovery of chronic patients is possible after robot-aided therapy. However, no consistent effect on the improvement in functional abilities has been reported. Restoration of motor control appears greater after robot-aided therapy than conventional therapy. We could not establish which aspects of robot-aided therapy (e.g., increased intensity of movements, most effective training modalities) were most responsible for the beneficial influence on recovery.

The clinical relevance of our findings is that robot-aided therapy is a promising new approach to rehabilitation of upper-limb motor control after stroke. For both sub-acute and chronic stroke patients, robot-aided therapy can improve motor control of the hemiparetic upper limb, perhaps even more than conventional therapy. 


\section{References}

1. Broeks JG, Lankhorst GJ, Rumping K, Prevo AJH. The long-term outcome of arm function after stroke: results of a follow-up study. Disabil Rehabil 1999;21(8):357364

2. Ingall T. Stroke - incidence, mortality, morbidity and risk. J Insur Med 2004; 36(2):143-152

3. Limburg M, Hijdra A, Cools HJM. Cerebrovascular disease [In Dutch: Cerebrovasculaire aandoeningen]. Houten: Bohn Stafleu Van Loghum; 1999

4. Barreca S, Wolf SL, Fasoli S, Bohannon R. Treatment interventions for the paretic upper limb of stroke survivors: a critical review. Neurorehabil Neural Repair 2003; 17:220-226

5. Feys HM, De Weerdt WJ, Selz BE, et al. Effect of a therapeutic intervention for the hemiplegic upper limb in the acute phase after stroke. A single-blind, randomized, controlled multicenter trial. Stroke 1998;29:785-792

6. Kwakkel G, Wagenaar RC, Twisk JWR, Lankhorst GJ, Koetsier JC. Intensity of leg and arm trai-ning after primary middle-cerebral-artery stroke: a randomised trial. Lancet 1999;354:189-194

7. Volpe BT, Ferraro M, Krebs HI, Hogan N. Robotics in the rehabilitation treatment of patients with stroke. Curr Atheroscler Rep 2002;4(4):270-276

8. Lum P, Reinkensmeyer D, Mahoney R, Rymer WZ, Burgar C. Robotic devices for movement therapy after stroke: current status and challenges to clinical acceptance. Top Stroke Rehabil 2002;8(4):40-53

9. Krebs HI, Hogan N, Volpe BT, Aisen ML, Edelstein L, Diels C. Overview of clinical trials with MIT-MANUS: a robot-aided neuro-rehabilitation facility. Technol Health Care $1999 ; 7(6): 419-423$

10. Reinkensmeyer DJ, Kahn LE, Averbuch M, McKenna-Cole A, Schmit BD, Rymer WZ. Understanding and treating arm movement impairment after chronic brain injury: progress with the ARM guide. J Rehabil Res Dev 2000;37(6):653-562

11. Burgar CG, Lum PS, Shor PC, Machiel Van der Loos HF. Development of robots for reha-bilitation therapy: the Palo Alto VA/Stanford experience. J Rehabil Res Dev 2000;37(6):663-673

12. Hesse S, Schulte-Tigges G, Konrad M, Bardeleben A, Werner C. Robot-assisted arm trainer for the passive and active practice of bilateral forearm and wrist movements in hemiparetic subjects. Arch Phys Med Rehabil 2003;84(6):915-920

13. Coote S, Stokes E, Murphy B, and Harwin W. The effect of GENTLE/s robot-mediated therapy on the upper extremity dysfunction post stroke. In: Proceedings of the 8th International Conference on Rehabilitation Robotics (ICORR); 2003 Apr 22-25, Daejon, South Korea:59-61

14. Fanin C, Gallina P, Rossi A, Zanatta U, and Masiero S. NeRebot: a wire-based robot 
for neurorehabilitation. In: Proceedings of the 8th International Conference on Rehabilitation Robotics (ICORR); 2003 Apr 22-25, Daejon, South Korea:23-26

15. Toth A, Fazekas G, Arz G, Jurak M, and Horvath M. Passive robotic movement therapy of the spastic hemiparetic arm with REHAROB: report of the first clinical test and the follow-up system improvement. In: Proceedings of the 9th International Conference on Rehabilitation Robotics (ICORR); 2005 Jun 28 - Jul 1, Chicago (IL), USA:127-130

16. Dewald J, Ellis MD, Holubar BG, Sukal T, Acosta AM. The robot application in the rehabilitation of stroke patients. Abstract. Neurology \& Rehabilitation 2004;4:S7

17. Nef $T$ and Riener R. ARMin - Design of a novel arm rehabilitation robot. In: Proceedings of the 9th International Conference on Rehabilitation Robotics (ICORR); 2005 Jun 28 - Jul 1, Chicago (IL), USA:57-60

18. Arz G, Toth A, Fazekas G, Bratanov D, Zlatov N. Three-dimensional anti-spastic physiotherapy with the industrial robots of 'REHAROB'. Proceedings of the 8th International Conference on Rehabilitation Robotics (ICORR); 2003 Apr 22-25; Daejon, South Korea 2003;215-218

19. Riener R, Frey M, Nef T, Bernhardt M, and Colombo G. New developments in rehabilitation robotics. In: Proceedings of the IEEE Mechatronics and Robotics Conference; 2004 Sep 13-15, Aachen, Germany:1397-1402

20. Charles SK, Krebs HI, Volpe BT, Lynch D, and Hogan N. Wrist rehabilitation following stroke: initial clinical results. In: Proceedings of the 9th International Conference on Rehabilitation Robotics (ICORR); 2005 Jun 28 - Jul 1, Chicago (IL), USA:13-16

21. Furusho J, Koyanagi K, Imada Y, Fujii Y, Nakanishi K, Domen K, Miyakoshi K, Ryu U, Takenaka S, and Inoue A. A 3D rehabilitation system for upper limbs developed in a 5 -year NEDO project and its clinical testing. In: Proceedings of the 9th International Conference on Rehabilitation Robotics (ICORR); 2005 Jun 28 - Jul 1, Chicago (IL), USA:53-56

22. Colombo R, Pisano F, Micera S, Mazzone A, Delconte C, Carrozza MC, Dario P, and Minuco G. Upper limb rehabilitation and evaluation of stroke patients using robotaided techniques. In: Proceedings of the 9th International Conference on Rehabilitation Robotics (ICORR); 2005 Jun 28 - Jul 1, Chicago (IL), USA:515-518

23. Merians $A S$, Jack $D$, Boian $R$, et al. Virtual reality-augmented rehabilitation for patients following stroke. Phys Ther 2002;82(9):898-915

24. Kline $T$, Kamper $D$, and Schmit B. Control system for pneumatically controlled glove to assist in grasp activities. In: Proceedings of the 9th International Conference on Rehabilitation Robotics (ICORR); 2005 Jun 28 - Jul 1, Chicago (IL), USA:78-81

25. Mulas M, Folgheraiter M, and Gini G. An EMG-controlled exoskeleton for hand rehabilitation. In: Proceedings of the 9th International Conference on Rehabilitation Robotics (ICORR); 2005 Jun 28 - Jul 1, Chicago (IL), USA:371-374

26. Kottink AI, Oostendorp $\mathrm{J}$, Buurke JH, Nene AV, Hermens HJ, IJzerman MJ. The orthotic effect of functional electrical stimulation on the improvement of walking in 
stroke patients with a dropped foot: a systematic review. Artif Organs 2004; 28(6):577-586

27. Van Tulder MW, Assendelft WJJ, Koes BW, Bouter LM. Method guidelines for systematic reviews in the Cochrane Collaboration Back Review Group for spinal disorders. Spine 1997;22(20):2323-2330

28. Aisen ML, Krebs HI, Hogan N, McDowell F, Volpe BT. The effect of robot-assisted therapy and rehabilitative training on motor recovery following stroke. Arch Neurol 1997; 54(4):443-446

29. Krebs HI, Hogan N, Aisen ML, Volpe BT. Robot-aided neurorehabilitation. IEEE Trans Rehabil Eng 1998;6(1):75-87

30. Volpe BT, Krebs HI, Hogan N, Edelsteinn L, Diels CM, Aisen ML. Robot training enhanced motor outcome in patients with stroke maintained over 3 years. Neurology 1999;53(8):1874-1876

31. Volpe BT, Krebs HI, Hogan N, Edelstein OTR L, Diels C, Aisen M. A novel approach to stroke rehabilitation: robot-aided sensorimotor stimulation. Neurology 2000; 54(10): 1938-1944

32. Krebs HI, Volpe BT, Aisen ML, Hogan N. Increasing productivity and quality of care: robot-aided neuro-rehabilitation. J Rehabil Res Dev 2000; 37(6):639-652

33. Fasoli SE, Krebs HI, Stein J, Frontera WR, Hogan N. Effects of robotic therapy on motor impairment and recovery in chronic stroke. Arch Phys Med Rehabil 2003;84(4):477-482

34. Ferraro M, Palazzolo JJ, Krol J, Krebs HI, Hogan N, Volpe BT. Robot-aided sensorimotor arm training improves outcome in patients with chronic stroke. Neurology 2003;61(11):1604-1607

35. Fasoli SE, Krebs HI, Stein J, Frontera WR, Hughes R, Hogan N. Robotic therapy for chronic motor impairments after stroke: follow up results. Arch Phys Med Rehabil 2004;85:1106-1111

36. Stein J, Krebs HI, Frontera WR, Fasoli SE, Hughes R, Hogan N. Comparison of two techniques of robot-aided upper limb exercise training after stroke. Am J Phys Med Rehabil 2004;83(9):720-728

37. Krebs HI, Ferraro $\mathrm{M}$, Buerger $\mathrm{SP}$, et al. Rehabilitation robotics: pilot trial of a spatial extension for MIT-Manus. J Neuroengineering Rehabil 2004;1(1):5

38. Lum PS, Burgar CG, Shor PC, Majmundar M, Van der Loos M. Robot-assisted movement training compared with conventional therapy techniques for the rehabilitation of upper-limb motor function after stroke. Arch Phys Med Rehabil 2002;83(7):952-959

39. Lum PS, Burgar CG, Shor PC. Evidence for improved muscle activation patterns after retraining of reaching movements with the MIME robotic system in subjects with post-stroke hemiparesis. IEEE Trans Neur Syst Rehabil Eng 2004;12(2):186-194

40. Hesse S, Werner C, Pohl M, Rueckriem S, Mehrholz J, Lingnau ML. Computerized arm training improves the motor control of the severely affected arm after stroke: a 
single-blinded randomized trial in two centers. Stroke 2005;36(9): 1960-1966

41. Sutton AJ, Abrams KR, Jones DR, Sheldon TA, Song F. Methods for meta-analysis in medical research. Chichester, England: John Wiley \& sons; 2002: Chapter 3;37-56

42. Sutton AJ, Abrams KR, Jones DR, Sheldon TA, Song F. Methods for meta-analysis in medical research. Chichester, England: John Wiley \& sons; 2002: Chapter 4;57-72

43. Abrams KR, Lambert PC, Shaw C, Sansó B. Meta-analysis of heterogeneously reported study results: a Bayesian approach. In: Stangl DK, Berry DA, eds. Metaanalysis in medicine and health policy; New York, USA: Dekker; 2000: 29-63

44. Reinkensmeyer DJ, Emken JL, Cramer SC. Robotics, motor learning, and neurologic recovery. Annu Rev Biomed Eng 2004;6:497-525

45. Wagenaar RC, Meijer OG. Effects of stroke rehabilitation: a critical review of the literature. J Rehabil Sci 1991;4(3):61-73

46. Jørgenson HS, Nakayama $\mathrm{H}$, Raaschou HO, Vive-Larsen J, Støier M, Olsen TS. Outcome and time course of recovery in stroke. Part II: Time course of recovery. The Copenhagen Stroke Study. Arch Phys Med Rehabil 1995;76:406-412

47. Hendricks HT, Van Limbeek J, Geurts AC, Zwarts MJ. Motor recovery after stroke: a systematic review of the literature. Arch Phys Med Rehabil 2002;83:1629-1637

48. Cauraugh JH, Kim S. Two coupled motor recovery protocols are better than one: electromyogram-triggered neuromuscular stimulation and bilateral movements. Stroke 2002;33:1589-1594

49. Page SJ, Gater DR, Bach-y-Rita P. Reconsidering the motor recovery plateau in stroke rehabilitation. Arch Phys Med Rehabil 2004;85:1377-1381

50. Van der Lee JH, Snels IA, Beckerman H, Lankhorst GJ, Wagenaar RC, Bouter LM. Exercise therapy for arm function in stroke patients: a systematic review of randomized controlled trials. Clin Rehabil 2001;15:20-31

51. Platz T. Evidence-based rehabilitation of the arm. A systematic review [In German: Evidenzbasierte Armrehabilitation. Eine systematische Literaturübersicht]. Nervenarzt 2003;74:841-849

52. Gladstone DJ, Danells CJ, Black SE. The Fugl-Meyer assessment after stroke: a critical review of its measurement properties. Neurorehab Neural Repair 2002; 16(3):232-240

53. Reker DM, O'Donnell JC, Hamilton BB. Stroke rehabilitation outcome variation in Veterans Affairs rehabilitation units: accounting for case-mix. Arch Phys Med Rehabil 1998;79:751-757

54. Krebs HI, Celestino J, Williams D, Ferraro M, Volpe BT, Hogan N. A wrist extension to MIT-MANUS. In: Bien Z, Stefanov D, eds. Advances in human-friendly robotic technologies for movement assistance/movement restoration for people with disabilities; Springer-Verlag series lecture notes in control and information sciences. Berlin-Heidelberg: Springer-Verlag; 2004: chapter 24

55. Sanchez RJ, Wolbrecht E, Smith R, Liu J, Rao S, Cramer S, Rahman T, Bobrow JE, and Reinkensmeyer DJ. A pneumatic robot for re-training arm movement after 
stroke: rationale and mechanical design. In: Proceedings of the 9th International Conference on Rehabilitation Robotics (ICORR); 2005 Jun 28 - Jul 1, Chicago (IL), USA:500-504

56. Beer RF, Given JD, Dewald JPA. Task-dependent weakness at the elbow in patients with hemiparesis. Arch Phys Med Rehabil 1999;80:766-772

57. Beer RF, Dewald JPA, Rymer WZ. Deficits in the coordinaton of multijoint arm movements in patients with hemiparesis: evidence for disturbed control of limb dynamics. Exp Brain Res 2000;131:305-319

58. Beer RF, Dewald JPA, Dawson ML, Rymer WZ. Target-dependent differences between free and constrained arm movements in chronic hemiparesis. Exp Brain Res 2004; 156:458-470

59. Sukal TM, Ellis MD, and Dewald JPA. Dynamic characterization of upper limb discoordination following hemiparetic stroke. In: Proceedings of the 9th International Conference on Rehabilitation Robotics (ICORR); 2005 Jun 28 - Jul 1, Chicago (IL), USA:519-521

60. Dewald JPA, Pope PS, Given JD, Buchanan TS, Rymer WZ. Abnormal muscle coactivation patterns during isometric torque generation at the elbow and shoulder in hemiparetic subjects. Brain 1995;118:495-510

61. Kahn LE, Averbuch M, Rymer WZ, and Reinkensmeyer DJ. Comparison of robotassisted reaching to free reaching in promoting recovery from chronic stroke. In: Proceedings of the 7th International Conference on Rehabilitation Robotics (ICORR); 2001 Apr 25-27, Evry, France:39-44

62. De Kroon JR, IJzerman MJ, Chae J, Lankhorst GJ, Zilvold G. Relation between stimulation characteristics and clinical outcome in studies using electrical stimulation to improve motor control of the upper extremity in stroke. J Rehab Med 2005;37:6574

63. Martinsson L, Wahlgren NG, Hardemark HG. Amphetamines for improving recovery after stroke. Cochrane Database Syst Rev 2003;3:CD002090

64. Wolf SL, Blanton S, Baer H, Breshears J, Butler AJ. Repetitive task practice: A critical review of constraint-induced movement therapy in stroke. Neurol 2002;8:325-338

65. Lum PS, Taub E, Schwandt D, Postman M, Hardin P, Uswatte G . Automated constraint-induced therapy extension (AutoCITE) for movement deficits after stroke. J Rehabil Res Dev 2004;41:249-258

66. Taub E, Lum PS, Hardin P, Mark VW, Uswatte G. AutoCITE: automated delivery of CI therapy with reduced effort by therapists. Stroke 2005;36(6):1301-1304

67. Hesse S, Werner C. Poststroke motor dysfunction and spasticity: novel pharmacological and physical treatment strategies. CNS Drugs 2003;17(15):10931107

68. Pomeroy VM, Tallis RC. Physical therapy to improve movement performance and functional ability poststroke. Part 1. Existing evidence. Rev Clin Gerontol 2000; 10:261-290 
- Disorder

1. cerebrovascular accident (M)

2. CVA

3. stroke

4. hemiplegia (M)

5. hemipleg*

6. hemiparesis $(M)$

7. hemipare*

8. \#1 OR \#2 OR \#3 OR \#4 OR \#5 OR \#6 OR \#7

- Intervention

9. robotics (M)

10. robot*

11. \#9 OR \#10

- Body segment

12. upper extremity (M)

13. upper extremities

14. upper limb

15. upper limbs

16. arm

17. arms

18. \#12 OR \#13 OR \#14 OR \#15 OR \#16 OR \#17

- Combination robotics \& cerebrovascular accident

19. \#11 AND \#8

- Combination robotics \& upper extremity

20. \#11 AND \#18

limited to publication type: clinical trial

*This strategy provided the basis for adjustment to other databases $M$ indicates Mesh subject heading 


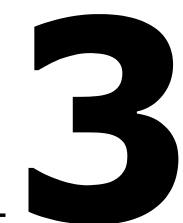

\title{
INFLUENCE OF GRAVITY COMPENSATION ON MUSCLE ACTIVITY DURING REACH AND \\ RETRIEVAL IN HEALTHY ELDERLY
}

\author{
GB Prange \\ LAC Kallenberg \\ MJA Jannink \\ AHA Stienen \\ $\mathrm{H}$ van der Kooij \\ MJ IJzerman \\ HJ Hermens
}

J Electromyogr Kinesiol 2009;19:e40-e49 


\section{Abstract}

Introduction - Arm support like gravity compensation may improve arm movements during stroke rehabilitation. It is unknown how gravity compensation affects muscle activation patterns during reach and retrieval movements. Since muscle activity during reach is represented by a component varying with movement velocity and a component supposedly counteracting gravity, we hypothesized that gravity compensation decreases the amplitude of muscle activity, but does not affect the pattern. To examine this, we compared muscle activity during well defined movements with and without gravity compensation in healthy elderly.

Methods - Ten subjects performed reach and retrieval movements with and without gravity compensation. Muscle activity of biceps, triceps, anterior, middle and posterior parts of deltoid and upper trapezius was compared between the two conditions.

Results - The level of muscle activity was lower with gravity compensation in all muscles, reaching significance in biceps, anterior deltoid and trapezius $(p=0.026)$. The muscle activation pattern did not differ between movements with and without gravity compensation $(\mathrm{p}=0.662)$.

Discussion - Gravity compensation only influenced the level of muscle activity but not the muscle activation pattern in terms of timing. Future studies should examine if the influence of gravity compensation is comparable for stroke patients. This may stimulate early and intensive training during rehabilitation. 


\section{Introduction}

A stroke can result in disturbed sensory and motor functions of contra-lateral body parts (i.e., hemiparesis), along with communicative and cognitive disorders. With respect to the upper extremity, impaired arm and hand function causes limitations in activities of daily living for the majority of stroke patients; only $5-20 \%$ of the patients experience full recovery, measured at 6 months post-stroke. ${ }^{1}$ Recovery of arm function after stroke can be stimulated by an intensive program of exercise therapy (i.e., frequent and/or extended sessions) that involves active movement of the affected arm. ${ }^{2-4}$

In clinical practice, active arm movements are frequently facilitated by supporting the weight of the arm, for example by suspending the arm from a frame overhead. Recently, technological innovations such as robotic devices are also applied to stimulate recovery of arm function after stroke. ${ }^{5}$ These devices often include arm support, to compensate for the effect of gravity on the arm. ${ }^{6}$ Research on arm support for stroke patients, using a frictionless air slide above a table, showed an increase in maximal voluntary torques of isometric elbow extension when the hemiparetic arm was supported against gravity. ${ }^{7}$ A subsequent study showed that the active range of elbow extension increased with arm support during reaching movements. ${ }^{8}$ This indicates that using arm support may be beneficial to improve arm movements in post-stroke rehabilitation, since patients do not have to use their remaining muscle strength and coordination to lift and hold their arm themselves.

Although for stroke patients some information about the influence of gravity compensation on kinetic and kinematic characteristics of reaching movements (like muscle torques, range of motion and movement velocity) is available, only little is known about the way gravity compensation influences muscle activity.

Research on the kinematics of goal-directed arm movements in micro-gravity during parabolic flights has indicated that gravity is implemented in internal models used in planning of movements by the central nervous system. ${ }^{9-11}$ Therefore, compensation of the influence of gravity on the arm may affect the muscle activation patterns of reaching movements. A study into differences between arm movements in different gravitational loads showed that different patterns of muscle activation occurred during movements with gravity and against gravity. ${ }^{12}$

On the other hand, muscle activity signals during forward-directed reach were found to be determined by two principal components, of which the contribution to 
the muscle activity signal depended on the weighting factor of each component. ${ }^{13}$ One 'phasic' component varied with movement velocity, the other 'tonic' component was unrelated to movement velocity and was assumed to be involved in counteracting gravity during reaching. The scaling of the phasic component with movement velocity was in line with the recorded muscle activity during reaching with different speeds. However, it was not verified whether the tonic component did relate to counteracting gravity. ${ }^{13}$

To examine this, the present study aimed to investigate the influence of gravity compensation on muscle activation patterns during reach and retrieval movements performed by healthy elderly. Based on the study by Flanders and Herrmann, ${ }^{13}$ we would expect that when the influence of gravity on the arm is compensated during reaching, the amplitude of muscle activity is affected, but the temporal pattern remains unchanged. 


\section{Methods}

\section{Subjects}

Ten healthy persons participated in this study, after providing written informed consent. Inclusion criteria were that the subjects were over 50 years of age and had no known history of neuromuscular, orthopedic or rheumatologic disorders of the upper extremity. The study was approved by the local medical ethics committee.

\section{Apparatus}

A mechanical, passive device was developed to counteract the influence of gravity on the upper extremity (named Freebal, derived from 'balanced freedom'). The device consisted of two independent springs, connected to pliable joint braces at the elbow and wrist via ropes and pulleys overhead (figure 3.1). This system provided a constant and individually adjustable amount of gravity compensation throughout the entire workspace of approximately $1 \mathrm{~m}^{3}$, enabling three dimensional movements. A more detailed description of the Freebal is published elsewhere. ${ }^{14,15}$

\section{Procedure}

The subjects performed two series of movements with the dominant arm, once with and once without gravity compensation. To reduce the potential effect of learning or adaptation, the subjects were assigned randomly to one of two

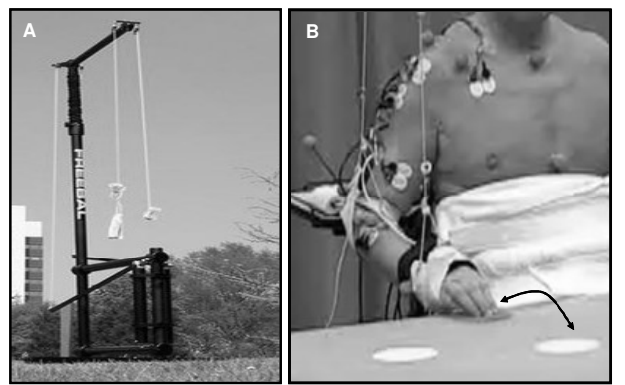

Figure 3.1 Apparatus for gravity compensation

Notes: (A) the Freebal device in full view; (B) close-up of the arm attached to the wrist and elbow straps of Freebal, with the hand on the starting dot and the arm in the initial posture; the arrow indicates the required movement from starting dot to target dot and back; also visible are EMG electrodes and reflective markers (see Kinemetic data recording for details). 
sequences, either performing the movements first with and subsequently without gravity compensation (group A), or in reversed order (group B).

The subjects were seated at an in height adjustable table and secured to the chair with straps to limit compensational trunk movements. Subjects sat with the upper arm parallel to the trunk, the elbow flexed $90^{\circ}$, the forearm in pronation and the wrist fixated in a neutral position midway between flexion/extension and radial/ulnar-abduction by a splint. In this position the hand was placed on the starting dot (figure 3.1). The target dot was placed $35 \mathrm{~cm}$ from the starting dot, so that the subjects reached forward in the sagittal plane using both the shoulder and the elbow. Both dots had a diameter of $10 \mathrm{~cm}$.

The subjects performed repeated multi-joint reach and retrieval movements during $30 \mathrm{~s}$, alternating between starting and target dot. The subjects were instructed to move at their own comfortable pace and touch the centre of the dots, placing more focus on accuracy than on velocity.

\section{Measurements and data recording}

\section{EMG recordings}

Bi-polar surface electromyography (EMG) was recorded using disc-shaped wet-gel Neuroline Ag/AgCl-electrodes (type 72000-S; Medicotest A/S, Ølstykke, Denmark) with a recording area of $95 \mathrm{~mm}^{2}$ and an inter-electrode distance of $2 \mathrm{~cm}$. Electrodes were placed over six superficial muscles, according to guidelines of the SENIAM project: long head of biceps (BIC); long head of triceps (TRI); anterior $(\mathrm{DA})$, middle (DM), posterior (DP) part of deltoid; upper part of trapezius (TRA). ${ }^{16}$ After differential amplification using a K-Lab amplifier (K-Lab, Haarlem, the Netherlands; input impedance $10 \mathrm{G} \Omega$, common mode rejection ratio $>110 \mathrm{~dB}$, input voltage noise $<2 \mu \mathrm{V}$, gain 18750), the EMG signals were high-pass filtered (third-order Butterworth filter, cut-off frequency $20 \mathrm{~Hz}$ ) and digitized by a 12-bit analog-to-digital converter with a sample rate of $1000 \mathrm{~Hz}$. These EMG signals were then band-pass filtered (second-order zero phase shift Butterworth, cut-off frequencies 20-400 Hz) and converted to smooth rectified EMG (SRE) signals (using a low-pass second-order zero phase shift Butterworth filter at $25 \mathrm{~Hz}$ for smoothing) for each muscle per subject.

\section{Kinematic data}

Changes in positions of arm segments were recorded using an infrared 3D-motion analysis system (VICON 370: six cameras, sample rate $50 \mathrm{~Hz}$; Oxford Metrics Ltd, 
Oxford, United Kingdom). Reflective markers were placed on 10 bony landmarks on the arm and trunk and converted to joint angles according to guidelines of the International Society of Biomechanics (ISB). ${ }^{17}$

The elbow joint angle was specified as the angle between humerus and forearm (maximal elbow flexion is $0^{\circ}$, maximal elbow extension is $180^{\circ}$ ). The shoulder joint orientation was described using two angles (see figure 3.2 for illustration). First, the plane of elevation was defined as the angle of the humerus with a virtual line through both shoulders, viewed in the transversal plane (outward/lateral is $0^{\circ}$, arm extended forward is $90^{\circ}$ ). In other words, it represented the angle of the projection of the upper arm on the horizontal plane. Second, the angle of elevation was the angle between humerus and trunk in the plane of elevation (consisting of the vertical plane through the upper arm), irrespective of the orientation of the humerus in the transversal plane (humerus parallel with trunk is $0^{\circ}$, humerus parallel with horizontal is $90^{\circ}$ ). These angles were low-pass filtered at $20 \mathrm{~Hz}$ with a second-order zero phase shift Butterworth filter and linearly interpolated from 50 to $1000 \mathrm{~Hz}$ to match the sample rate of the EMG recordings.

\section{Synchronous display of EMG and kinematics}

Custom written software using the Labview environment (National Instruments, Austin, TX, United States) was used to analyze SRE signals in relation to joint angles. Reversals in movement direction were defined at minimum and maximum elbow joint angles (hand on starting and target dot, respectively), determined by zero-crossings of the elbow angular velocity. A reach (minimal to maximal elbow angle) and subsequent retrieval (maximal to minimal elbow angle) movement constituted a movement cycle (see figure 3.3).
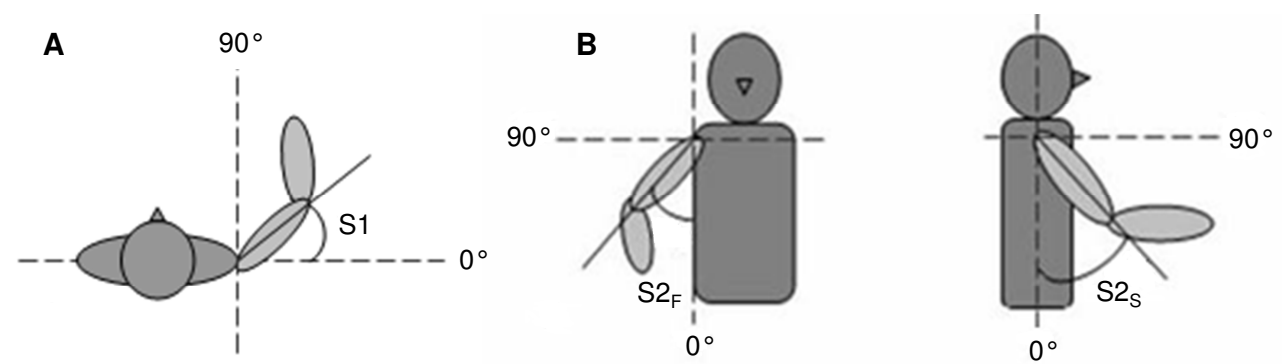

Figure 3.2 Definition of shoulder angles

Notes: (A) S1: plane of elevation, angle of the humerus with a virtual line through both shoulders; (B) S2: angle of elevation, angle between humerus and trunk in the plane of elevation (consisting of the vertical plane through the upper arm), represented in frontal view $\left(\mathrm{S2}_{\mathrm{F}}\right)$ and sagittal view $\left(\mathrm{S2}_{\mathrm{S}}\right)$. 


\section{Data analysis}

The EMG and joint angle data were visually inspected for missing data or recording errors. The SRE-values and joint angle trajectories were averaged over all repeated movement cycles of one $30 \mathrm{~s}$ series of movements to obtain an average muscle activation pattern (MAP), which was normalized to the average duration of all repeated movement cycles. The reach and retrieval trajectories were each divided in three movement parts: part (I) initiation of movement, defined by $1-20 \%$ of a trajectory; part (II) steady state movement during the middle part of a trajectory, from $31 \%$ to $70 \%$; part (III) termination of movement, consisting of $81-100 \%$

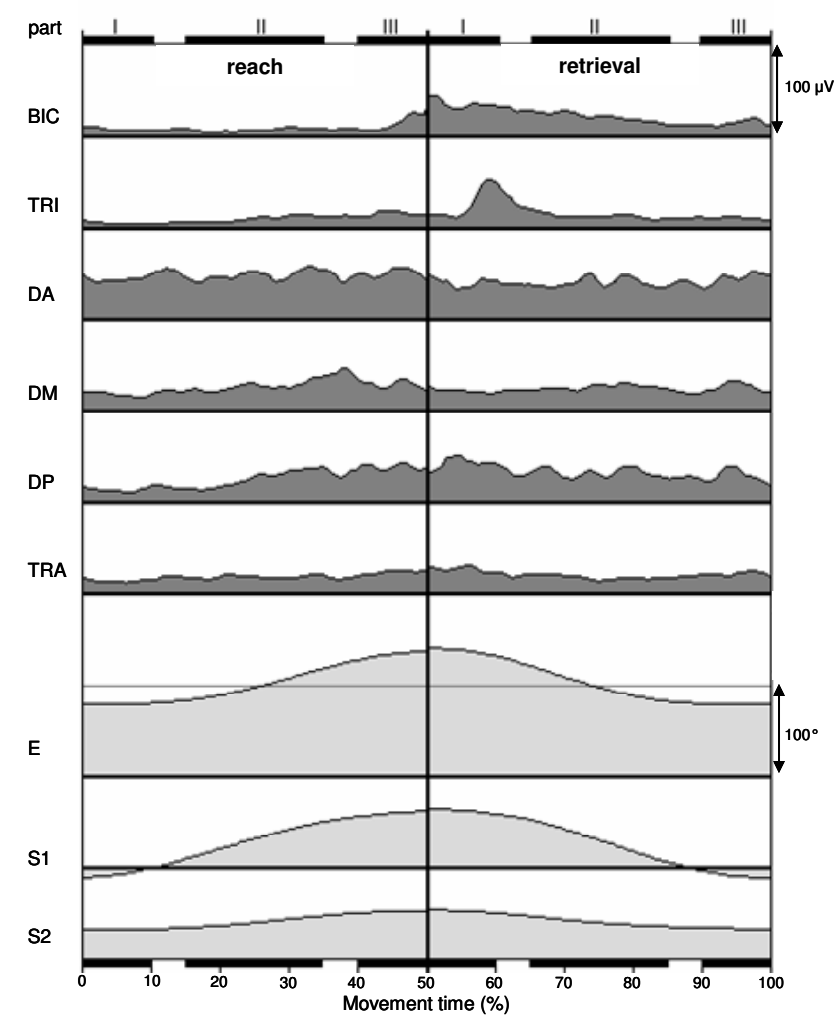

Figure 3.3 Muscle activation pattern (MAP) of 6 muscles and corresponding joint angles, during movement without gravity compensation (time in \% of averaged cycle duration) of one subject, including definition of movement parts by black bars

Notes: part I $=$ initiation, $\mathrm{II}=$ steady state, $\mathrm{III}=$ termination of movement; $\mathrm{BIC}=\mathrm{m}$. biceps brachii long head; TRI $=\mathrm{m}$. triceps brachii long head; $\mathrm{DA}=\mathrm{m}$. deltoideus anterior; $\mathrm{DM}=\mathrm{m}$. deltoideus medialis; $\mathrm{DP}=\mathrm{m}$. deltoideus posterior; $\mathrm{TRA}=\mathrm{m}$. trapezius pars decendens; $\mathrm{E}=$ elbow flexion $/$ extension angle; $\mathrm{S} 1$ = shoulder plane of elevation; $\mathrm{S} 2$ = shoulder angle of elevation. 
(see figure 3.3). Per subject, the SRE-values of each MAP were averaged within each movement part as a measure of level of muscle activity.

To quantify movements, several parameters were derived from kinematic data: movement time was defined as the movement cycle duration averaged over all movement cycles per subject (in ms); movement symmetry was defined as the relative duration of reach with respect to the average movement cycle (in \%); and joint excursions of elbow and shoulder angles were defined as the difference between minimal and maximal joint angles (in ${ }^{\circ}$ ), averaged over all movement cycles.

\section{Statistical analysis}

Initially, SRE-values were transformed to LN(SRE)-values to ensure normal distribution of residuals. To detect potential effects of learning or adaptation, muscle activity and movement performance parameters of the two groups performing movements with and without gravity compensation in reversed order ( $A$ and $B$ ) were compared using a t-test for independent samples in the case of normally distributed parameters and a non-parametric equivalent, the Kolmogorov-Smirnov test, for parameters deviating from the normal distribution. Furthermore, either paired-samples t-tests or the non-parametric equivalent, Wilcoxon signed ranks tests, were used to examine differences in movement execution parameters (movement time, movement symmetry and joint excursions) due to gravity compensation. For all these tests the significance level was 0.05 .

Linear mixed modeling techniques (i.e., multilevel linear modeling, random coefficient analysis) were applied to test differences in SRE-values due to gravity compensation (two-level factor ' $f z-c o m p '$ ') in each muscle (six-level factor 'muscle') per movement part (three-level factor 'part'), separately for reach and retrieval. To account for the correlation of repeated measurements within one subject, the factor 'subject' was included as a random factor. The factors ' $f z-c o m p '$ ', 'muscle' and 'part' were treated as fixed effects, because the effect of gravity compensation on muscle activity was similar for all subjects. The two-way interactions 'muscle $x$ part', 'fz-comp x muscle' and 'fz-comp x part' were included to examine the change of muscle activity across movement parts per muscle, the difference of the influence of gravity compensation on the level of muscle activity between muscles and the influence of gravity compensation on the change of muscle activity across movement parts, respectively. For all significant effects and interactions post-hoc tests (Sidak adjustment) were performed. The level of significance was defined as 0.05 . 


\section{Results}

\section{Subjects}

Data of all 10 subjects were used for analysis, of which average values of physical characteristics are presented in table 3.1. Regarding the order of conditions, seven subjects moved first with and next without gravity compensation (group A), while three subjects performed the test in the reversed order (group B). There were no significant differences in physical characteristics, movement execution parameters and SRE-values between the two groups, so data of all subjects were pooled in subsequent analyses.

Table 3.1 Physical characteristics of 10 healthy elderly

\begin{tabular}{l|l}
\hline & Subjects $(\mathbf{n = 1 0})$ \\
\hline Sex (male/female)* & $5 / 5$ \\
Arm dominance $($ right/left)* & $10 / 0$ \\
Age $(\text { years })^{\dagger}$ & $65.9( \pm 6.4)$ \\
Height $(\mathrm{m})^{\dagger}$ & $1.74( \pm 0.12)$ \\
Weight $(\mathrm{kg})^{\dagger}$ & $77.7( \pm 13.6)$ \\
Body Mass Index $\left(\mathrm{kg} / \mathrm{m}^{2}\right)^{\dagger}$ & $25.7( \pm 2.9)$ \\
\hline$*$ absolute numbers; ${ }^{\dagger}$ mean $^{\dagger} \mathrm{SD}$
\end{tabular}

Table 3.2 Movement execution parameters (mean \pm SD) and corresponding inter-subject coefficients of variation (COV = SD / mean) for movements without and with gravity compensation of 10 subjects

\begin{tabular}{l|ll}
\hline & WITHOUT compensation & WITH compensation \\
\hline elbow excursion $\left(^{\circ}\right)$ & $60.4( \pm 4.4)$ & $61.2( \pm 6.6)$ \\
inter-subject COV & 0.07 & 0.11 \\
shoulder plane excursion $\left(^{\circ}\right)$ & $81.6( \pm 11.7)$ & $78.2( \pm 11.9)$ \\
inter-subject COV & 0.14 & 0.15 \\
shoulder elevation excursion $\left(^{\circ}\right)^{*}$ & $23.2( \pm 9.5)$ & $18.8( \pm 6.8)$ \\
inter-subject COV & 0.41 & 0.36 \\
movement time $(\mathrm{ms})$ & $1327( \pm 383)$ & $1491( \pm 211)$ \\
inter-subject COV & 0.29 & 0.14 \\
movement symmetry $(\%)$ & $56.8( \pm 3.7)$ & $58.8( \pm 1.7)$ \\
inter-subject COV & 0.07 & 0.03 \\
\hline * significant difference between movement with and without gravity compensation $(\mathrm{p}<0.05)$
\end{tabular}




\section{Movement execution}

Movement execution parameters averaged over all subjects are presented in table 3.2. On average, $19( \pm 8)$ movement cycles per subject were analyzed. Comparison of movement execution with and without gravity compensation showed that movement time and movement symmetry were not significantly different $(p=0.224, p=0.160$, respectively). The joint excursions of elbow and shoulder plane were also comparable for both conditions $(p=0.376$ and $p=0.102$, respectively), but the excursion of shoulder elevation was somewhat smaller with gravity compensation $(p=0.013)$. However, the difference in shoulder elevation excursion was not even $5^{\circ}$, which was not regarded as a substantial difference in movement execution.

\section{Generic aspects of muscle activation}

In figure 3.3 a typical example of a MAP of movement without gravity compensation is displayed, which revealed some features in this subject. Most muscles were active throughout the movement, without periods of relative rest. An exception is TRI, which had very low levels of activity during reach and retrieval (except at the start of retrieval in this particular subject).
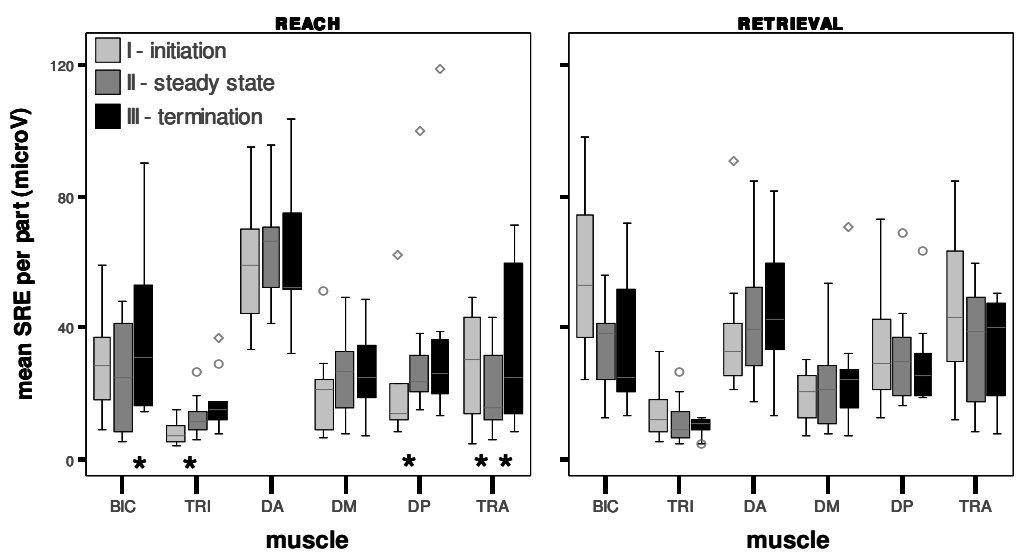

Figure 3.4 Median and interquartile ranges of SRE-values of 10 subjects per movement part for each muscle during movement without gravity compensation, for reach and retrieval separately

Notes: Muscle activity significantly increased during reach and significantly decreased during retrieval; muscle-specific changes in SRE-values from one part to next during reach are represented by asterisks $(* \mathrm{p}<0.05)$. Circles $(\circ)$ represent outliers (deviation of $<1.5$ times interquartile range) and diamonds $(\diamond)$ represent extremes (deviation of $<3$ times interquartile range). 
When comparing MAPs of movements without gravity compensation across subjects (figure 3.4), several generic aspects could be identified. Most muscles had persistent levels of muscle activity throughout the reach and retrieval movements, except that almost all subjects showed rather low levels of TRI activity. Furthermore, most muscles showed increasing SRE-values from movement initiation to termination during reach, as supported by a significant main effect of 'part' $(p=0.000)$. This general increase in SRE-values differed between muscles, as revealed by a significant interaction of 'muscle $x$ part' $(p=0.000)$. Post-hoc analyses (represented by asterisks in figure 3.4) showed increases in SRE-values from steady state to termination in BIC $(p=0.008)$ and from initiation to steady state in TRI $(p=0.000)$ and in DP $(p=0.001)$, while in TRA SRE-values decreased from initiation to steady state $(p=0.036)$ and subsequently increased $(p=0.033)$. During retrieval, a general decrease in muscle activity was observed towards movement termination (main effect of 'part'; $p=0.009$ ), which was not significantly different between muscles ('muscle $x$ part' $p=0.106$ ).

When integrating the qualitative and quantitative findings, generic aspects of muscle activation patterns could be identified for reach and retrieval without gravity compensation. BIC was active to lift and hold the lower arm above the table and aid in anteflexion of the shoulder. Muscle activity of TRI was very low, sometimes nearly absent, contributing to extension of the elbow towards the
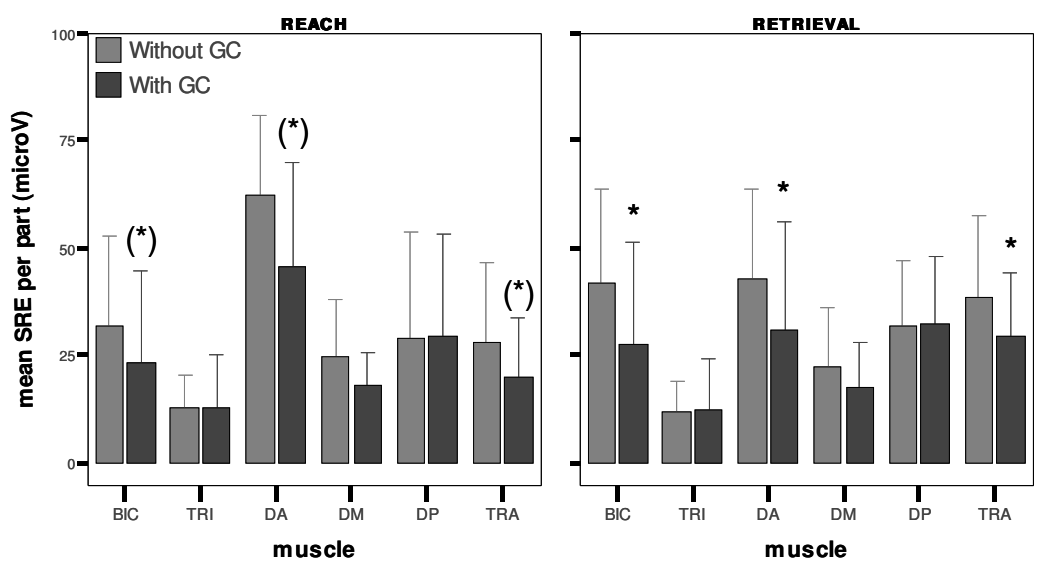

Figure 3.5 Comparison of mean ( \pm SD) SRE-values, averaged over all 3 parts per muscle, between movement without (light bars) and with (dark bars) gravity compensation (GC), for reach and retrieval separately $(n=10)$

Notes: Asterisks represent significant differences $(p<0.05)$. 
target. DA and DM were active to maintain a certain degree of shoulder abduction, to anteflex the shoulder during reach and to decelerate retroflexion during retrieval. Activity of DP decelerated anteflexion during reach and retroflexed the shoulder during retrieval. TRA was active to elevate the arm at the start of reach and retrieval and to position the scapula appropriately during the movements.

\section{Influence of gravity compensation on muscle activation}

Comparison of MAPs with and without gravity compensation (figure 3.5) showed a reduction in the level of activity of most muscles with gravity compensation, as underlined by a significant overall effect of ' $f z-c o m p '(p=0.000)$ for both reach and retrieval. During retrieval, the influence of gravity compensation differed between muscles as indicated by a significant interaction ' $f z$-comp $x$ muscle' $(p=0.007)$.
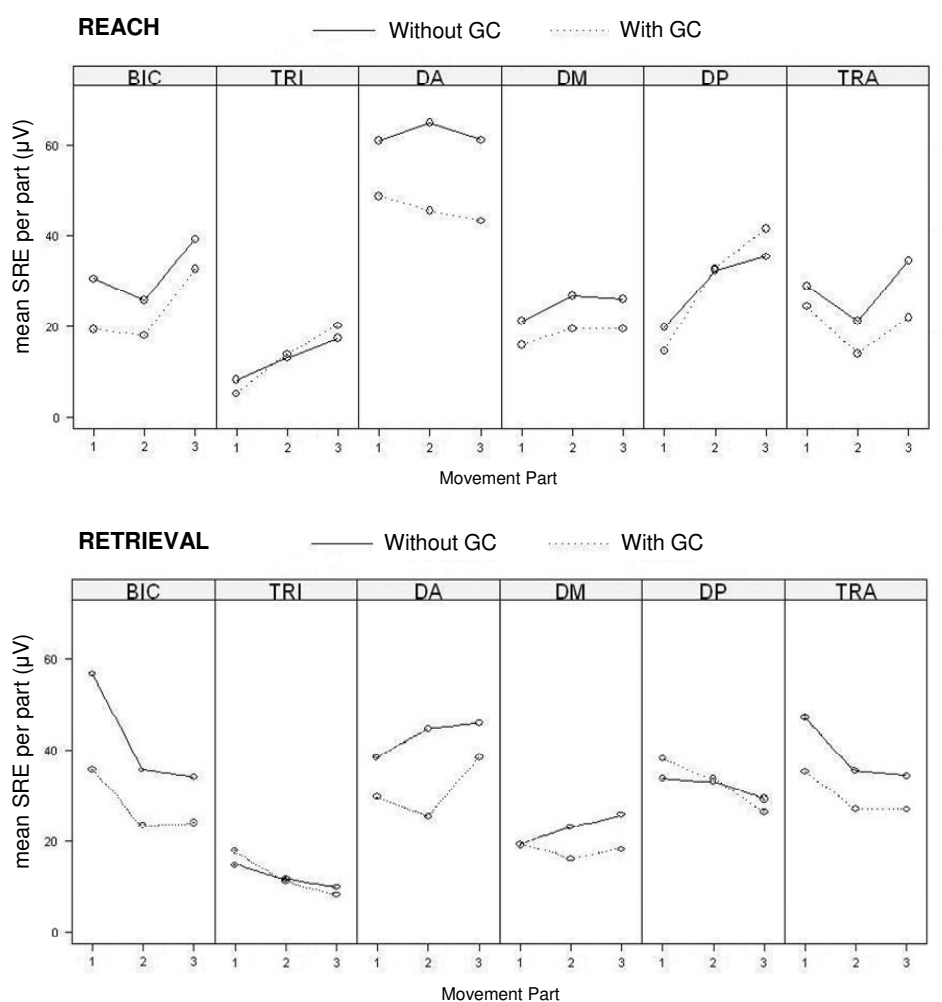

Figure 3.6 Changes in mean SRE-values across movement parts

Notes: comparison for movements without (solid lines) and with (dotted lines) gravity compensation (GC) of 10 subjects, per muscle and for reach and retrieval separately. 
Post-hoc differences (in figure 3.5 accentuated by asterisks) between movements with and without gravity compensation were found in BIC $(p=0.000)$, DA $(p=0.000)$ and TRA $(p=0.026)$. These same muscles also showed significant posthoc differences between the two conditions during reach $(p<0.007)$, but these differences were smaller than during retrieval, resulting in a non-significant interaction of 'fz-comp $x$ muscle' $(p=0.252)$ during reach.

Despite differences in the level of activity, the change of muscle activity across movement parts (figure 3.6) was similar for movements with and without gravity compensation for most muscles, as supported by a non-significant interaction ' $f z$ comp $x$ part' for both reach and retrieval $(p=0.662$ and $p=0.826$, respectively). Although TRI and DP displayed a deviating pattern with a slightly larger level of muscle activity at the end of reach and start of retrieval with gravity compensation, these differences were too small to result in a significant effect. 


\section{Discussion}

The aim of the present study with healthy elderly was to investigate the influence of gravity compensation on muscle activation patterns during reach and retrieval movements. A common aspect of muscle activity during movements without gravity compensation across all subjects was that most muscles show persistent activity during the entire reach and retrieval trajectories. As an exception, activity was very low in TRI. In general, the persistent activity increases during reach and decreases during retrieval. A plausible explanation is that larger torques act on the extended arm at the target dot then when the arm is close to the trunk at the starting dot, requiring a higher level of muscle activity at the end of reach and start of retrieval.

The generic aspect of persistent activity during reach and retrieval corresponds largely with the findings of Sabatini et al., who investigated 'natural' unsupported reaching movements with a similar arm orientation (shoulder abduction of approximately $45^{\circ}$, hand at table height) as the present study. ${ }^{18}$ They found that all recorded muscles (BIC, TRI, DA, DP and TRA) were active throughout the movement, with additional activity on top of this persistent activity in BIC, DP and TRA.

In contrast, several other studies described an alternating pattern of BIC/TRI and $D A / D P$ activity during reach and retrieval with arm support, interspersed with periods of relative rest. ${ }^{19-21}$ This discrepancy is probably related to the orientation of the arm during movements. In the present study the targets were at table height, requiring a certain amount of arm elevation against gravity to reach the target, while in the reported literature both the targets and the arm were in the horizontal plane at shoulder height (approximately $90^{\circ}$ shoulder elevation, with arm support) and no movement against gravity was necessary. A study examining timing of muscle activity for movements in different directions reported that singlejoint elbow movements against gravity resulted in periods of simultaneous activity of agonist and antagonist (i.e. co-activation), while movements in the horizontal plane produced an alternating pattern of agonist-antagonist activity. ${ }^{19}$ Thus, phasic activity may have been largely masked by a higher level of persistent activity during movements in the present study, requiring movement against gravity.

Besides arm orientation, other differences between the present study and the reported studies may also play a role. ${ }^{19-21}$ For example, in the present study movement velocity was lower, especially with respect to the study by Gribble et al. 
where subjects were instructed to make movements as fast as possible. ${ }^{21}$ Several studies have indicated that a higher movement velocity is related to a higher intensity of muscle activity, ${ }^{22,23}$ and to the presence of a typical tri-phasic pattern of agonist/antagonist activity. ${ }^{24,25}$ In addition, the present study included older subjects than several other studies, ${ }^{20,21}$ whom have been reported to have a lower depth of muscle activity modulation, meaning that in elderly the range from minimal to maximal values of generated muscle activity is smaller. ${ }^{26}$

Comparison of muscle activation patterns during reach and retrieval movements with and without gravity compensation showed that the level of muscle activity was lower with gravity compensation, while no substantial differences in movement execution between the two conditions were found. This difference was observed specifically in the muscles that counteract gravity during forward reach and retrieval movements: BIC, DA and TRA. The change of muscle activity across movement parts did not differ between the two conditions.

The observed decrease in the level of muscle activity without differences in the pattern of muscle activation confirmed our hypothesis, based on a study by Flanders and Herrmann suggesting that tonic muscle activity was related to counteracting gravity while phasic activity was not. ${ }^{13}$ These findings indicate that performing movements without the effect of gravity on the arm only reduced the level of muscle activity and did not affect the pattern of muscle activation in terms of timing.

Another study investigating a basic application of gravity compensation, manual support of gross arm movements in healthy persons, compared an unspecified parameter of muscle activity during supported arm elevations with unsupported movements. This study reported less muscle activity of the serratus anterior and the pectoralis major muscles during supported movements. ${ }^{27}$ Despite the use of other movements and muscles and a lack of specificity on analysis of muscle activity, this outcome is along the same lines of the influence of gravity compensation observed in the present study.

Remarkably, the application of gravity compensation did not lead to unmasking of phasic components, but a certain degree of tonic activity remained during movements with gravity compensation. This may be related to the many degrees of freedom that characterize upper extremity movements and the interaction torques acting on a joint due to movements of an adjacent joint. Simultaneous application of both agonist and antagonist activity can stabilize the joints and control the interaction torques during movements. ${ }^{20,21}$ This co-activation presents as 'tonic' activity during arm movements, even when the arm is supported. 
The findings of the present study show that healthy elderly performed similar movements with a lower level of muscle activity, while the change of muscle activity across movement parts did not differ during movements with gravity compensation. This indicates that when performing movements while the arm is supported against gravity only the level of muscle activity is influenced, but the pattern of muscle activation in terms of timing is not affected, compared to movements without support.

Moreover, this implies that, if the influence of gravity compensation on movements of stroke patients is comparable to healthy elderly, gravity compensation may be able to facilitate arm movements during post-stroke rehabilitation. The application of gravity compensation would not be interfering with the temporal characteristics of muscle activation, which are often impaired in stroke patients. ${ }^{28}$ In this way, patients would be able to repeat more movements and/or attend longer or more frequent sessions (i.e., higher training intensity) than in a situation without gravity compensation, which is beneficial in post-stroke rehabilitation. ${ }^{3}$ Future studies should further examine this potential of gravity compensation for stroke rehabilitation. 


\section{References}

1. Kwakkel G, Kollen BJ, Van der Grond J, Prevo AJH. Probability of regaining dexterity in the flaccid upper limb: impact of severity of paresis and time since onset in acute stroke. Stroke 2003;34:2181-2186

2. Feys HM, De Weerdt WJ, Selz BE, et al. Effect of a therapeutic intervention for the hemiplegic upper limb in the acute phase after stroke. A single-blind, randomized, controlled multicenter trial. Stroke 1998;29:785-792

3. Kwakkel G, Wagenaar RC, Twisk JWR, Lankhorst GJ, Koetsier JC. Intensity of leg and arm training after primary middle-cerebral-artery stroke: a randomised trial. Lancet 1999;354:189-194

4. Barreca S, Wolf SL, Fasoli S, Bohannon R. Treatment interventions for the paretic upper limb of stroke survivors: a critical review. Neurorehabil Neural Repair 2003;17:220-226

5. Prange GB, Jannink MJA, Groothuis CGM, Hermens $\mathrm{HJ}$, IJzerman MJ. A systematic review of the effect of robot-aided therapy on recovery of the hemiparetic arm after stroke. J Rehabil Res Dev 2006;43(2):171-184

6. Johnson MJ. Recent trends in robot-assisted therapy environments to improve reallife functional performance after stroke. J Neuroengineering Rehabil 2006;3:29

7. Beer RF, Given JD, Dewald JPA. Task-dependent weakness at the elbow in patients with hemiparesis. Arch Phys Med Rehabil 1999;80:766-772

8. Beer RF, Dewald JPA, Dawson ML, Rymer WZ. Target-dependent differences between free and constrained arm movements in chronic hemiparesis. Exp Brain Res 2004; 156:458-470

9. Smetanin BN, Popov KE. Effect of body orientation with respect to gravity on directional accuracy of human pointing movements. Eur J Neurosci 1997;9:7-11

10. Papaxanthis C, Pozzo T, Popocv KE, McIntyre J. Hand trajectories of vertical arm movements in one-G and zero-G environments. Evidence for a central representation of gravitational force. Exp Brain Res 1998;120(4):496-502

11. Pozzo T, Papaxanthis C, Stapley P, Berthoz A. The sensorimotor and cognitive integration of gravity. Brain Res Brain Res Rev 1998;28(1-2):92-101

12. Virji-Babul N, Cooke JD, Brown SH. Effects of gravitational forces on single joint arm movements in humans. Exp Brain Res 1994;99(2):338-346

13. Flanders M, Herrmann U. Two components of muscle activation: Scaling with the speed of arm movement. J Neurophysiol 1992;67(4):931-943

14. Stienen AHA, Van der Helm FCT, Prange GB, Jannink MJA, and Van der Kooij $H$. Effects of gravity compensation on the range-of-motion of the upper extremities in robotic rehabilitation after stroke. In: Proc Inter Shoulder Group; 2006 Oct 9-10, Chicago (IL), USA

15. Stienen AHA, Hekman EEG, Van der Helm FCT, Prange GB, Jannink MJA, Aalsma 
AMM, and Van der Kooij H. Freebal: dedicated gravity compensation for het upper extremities. In: Proceedings of the 10th International Conference on Rehabilitation Robotics (ICORR); June 13-15, 2007, Noordwijk aan Zee, the Netherlands:804-808

16. Hermens $\mathrm{HJ}$, Freriks $B$, Merletti $\mathrm{R}$, et al. European recommendations for surface electromyography, results of the SENIAM project. Enschede: Roessingh Research and Development; 1999

17. Wu G, Van der Helm FC, Veeger HE, et al. ISB recommendation on definitions of joint coordinate systems of various joints for the reporting of human joint motion Part II: shoulder, elbow, wrist and hand. J Biomech 2005;38(5):981-992

18. Sabatini AM. Identification of neuromuscular synergies in natural upper-arm movement. Biol Cybern 2002;86:253-262

19. Karst GM, Hasan Z. Timing and magnitude of electromyographic activity for two-joint arm movements in different directions. J Neurophysiol 1991;66(5):1594-1604

20. Dounskaia N, Ketcham CJ, Stelmach GE. Commonalities and differences in control of various drawing movements. Exp Brain Res 2002;146:11-25

21. Gribble PL, Mullin LI, Cothros N, Mattar A. Role of cocontraction in arm movement accuracy. J Neurophysiol 2003;89:2396-2405

22. Buneo CA, Soechting JF, Flanders M. Muscle activation patterns for reaching: The representation of distance and time. J Neurophysiol 1994;71(4):1546-1558

23. Gabriel DA. Shoulder and elbow muscle activity in goal-directed arm movements. Exp Brain Res 1997;116:359-366

24. Brown JM, Gilleard W. Transition from slow to ballistic movement: development of triphasic electromyogram patterns. Eur J Appl Physiol Occup Physiol 1991;63(5):381386

25. Berardelli $A$, Hallett $M$, Rothwell JC, et al. Single-joint rapid arm movements in normal subjects and in patients with motor disorders. Brain 1996;119:661-674

26. Ketcham CJ, Dounskaia NV, Stelmach GE. Age-related differences in the control of multijoint movements. Motor Control 2004;8:422-436

27. Hiengkaew V, Wichaiwong $\mathrm{K}$, Chaiyakul S, Deesin A. Concerning the pectoralis major in active reaching exercise. Electromyogr Clin Neurophysiol 2003;43:157-163

28. Chae J, Yang G, Park BK, Labatia I. Delay in initiation and termination of muscle contraction, motor impairment, and physical disability in upper limb hemiparesis. Muscle Nerve 2002;25:568-575 

INFLUENCE OF GRAVITY COMPENSATION ON MUSCLE ACTIVATION PATTERNS DURING DIFFERENT TEMPORAL PHASES OF ARM MOVEMENTS OF STROKE PATIENTS

GB Prange

MJA Jannink

AHA Stienen

$\mathrm{H}$ van der Kooij

MJ IJzerman

HJ Hermens

Neurorehabil Neural Rep 2009;23(5):478-485 


\section{Abstract}

Background - Arm support to help compensate for the effects of gravity may improve functional use of the shoulder and elbow during therapy after stroke, but gravity compensation may alter motor control.

Objective - To obtain quantitative information on how gravity compensation influences muscle activation patterns during functional, 3-dimensional reaching movements.

Methods - Eight patients with mild hemiparesis performed 2 sets of repeated reach and retrieval movements, with and without unloading the arm, using a device that acted at the elbow and forearm to compensate for gravity. Electromyographic (EMG) patterns of 6 upper extremity muscles were compared during elbow and shoulder joint excursions with and without gravity compensation.

Results - Movement performance was similar with and without gravity compensation. Smooth rectified EMG (SRE) values were decreased from $25 \%$ to $50 \%$ during movements with gravity compensation in 5 out of 6 muscles. The variation of SRE values across movement phases did not differ across conditions.

Conclusions - Gravity compensation did not affect general patterns of muscle activation in this sample of stroke patients, probably since they had adequate function to complete the task without arm support. Gravity compensation did facilitate active arm movement excursions without impairing motor control. Gravity compensation may be a valuable modality in conventional or robot-aided therapy to increase the intensity of training for mildly impaired patients. 


\section{Introduction}

A majority of patients have impaired arm and hand function after a stroke, causing difficulties in activities of daily living. ${ }^{1}$ To achieve as much functional independence as possible, restoration of arm function is one of the main objectives of stroke rehabilitation. For optimal results, functional exercises with active participation of the patient are incorporated in an intensive and motivating training program. ${ }^{2-4}$

Improvement of functional use of the arm can be related to restoration of neural function within affected tissue and incorporation of pathways that may not usually contribute to reaching, as well as to behavioral adaptations and compensatory strategies. Specific interventions aimed at each of these potential mechanisms, however, have not been determined so that patients can obtain the best gains in arm function. ${ }^{5,6}$

Recent technological developments have led to the use of robotic devices for the application of active and intensive training for the affected arm. In a recent systematic review, we concluded that neurophysiologic aspects of arm function of stroke patients improve after robot-aided therapy, but the effect on functional use of the arm in activities of daily living is less distinct. ${ }^{7}$ Such limited generalization of training to increased functional use of the arm is not only noticed after robot-aided therapy, but also in many conventional interventions in stroke rehabilitation. ${ }^{2,8}$ More insight into the working mechanisms of individual training interventions is needed to allow better planning of the timing and content of rehabilitation. ${ }^{5}$

Concerning the content of robot-aided therapy, our systematic review indicated that different training modalities, such as passive movement, active movement with robotic assistance, and active movement with robotic resistance are applied simultaneously in a robot-aided training session. Besides these training modalities, arm support is incorporated in the design of many robotic devices to compensate for the influence of gravity on the arm. ${ }^{9}$ However, the individual contribution of each of those modalities to the improvement of arm function after robot-aided therapy is largely unknown. ${ }^{7}$

Regarding the contribution of arm support to restoration of arm function, initial results of an exercise therapy program applying gravity compensation by sling suspension showed an improvement of arm function of stroke patients after 9 weeks of training. ${ }^{10}$ This suggests that the application of gravity compensation alone may be a valuable tool to stimulate functional improvement in post-stroke rehabilitation. 
Research into the instantaneous influence of gravity compensation on arm movements after stroke has been mainly focused on kinematics of arm movements. Beer and collegues showed that the active range of elbow extension increases during 2-dimensional planar reaching movements at shoulder height with arm support when compared to similar unsupported movements. ${ }^{11}$ Results along the same lines were found during reaching movements in more functional circumstances. Maximal reaching distance during a 3-dimensional movement, starting with the hand at waist height and reaching to a target at shoulder height, as if reaching for a cup in a drawer, is slightly larger when gravity compensation is applied to the arm of stroke patients. ${ }^{12}$

Insight into underlying working mechanisms of the influence of gravity compensation on arm function can be obtained by studying muscle activation patterns. In previous research, we found that in healthy persons the level of muscle activity in muscles counteracting gravity during reaching movements decreased with gravity compensation. ${ }^{13}$ At the same time, gravity compensation did not affect the general pattern of muscle activity during functional, 3dimensional reaching movements at table height, ${ }^{13}$ even though the gravitational force is taken into account in internal models when planning and executing movements. ${ }^{14}$

However, after stroke, limitations in motor planning, integration of sensorimotor information, generation, and coordination of muscle activity, both within and between muscles, may reduce functional use of the arm. ${ }^{15}$ Therefore, it is unclear how the application of gravity compensation affects motor control of arm movements in stroke patients. In the light of the increased range of motion with arm support, ${ }^{11,12}$ we expect that gravity compensation may influence motor control of stroke patients positively by inducing changes in muscle activation patterns. These changes may suggest a potential for neurological recovery in the case of more normal muscle activation patterns, or may indicate that behavioral adaptation takes place in the case of new patterns of muscle activation. Insight into the nature and direction of changes in motor control due to gravity compensation is essential to determine optimal application of this intervention in stroke rehabilitation in terms of content and timing.

Therefore, the objective of the present explorative study was to obtain quantitative information on how gravity compensation influences muscle activation patterns of stroke patients during functional, 3-dimensional reaching movements. 


\section{Methods}

\section{Participants}

After selection by a rehabilitation specialist, 10 stroke patients were recruited from a local rehabilitation center. Inclusion criteria for participation were that stroke patients had to be: (1) at least 4 weeks post-stroke; (2) able to lift their arm (partly) against gravity; (3) free from additional orthopedic, neurological, or rheumatologic disease of the upper extremities; (4) not suffering from shoulder pain either in rest or during movement; and (5) able to understand and follow instructions. Patients provided written informed consent before being admitted to the study. The medical ethics committee of the institution approved the study.

\section{Procedure}

The current arm function of each participant was assessed at the start of the experiment using the upper extremity portion of the Fugl-Meyer assessment (maximal score of 66). ${ }^{16}$ Participants performed 2 movement series with the affected arm, once with and once without gravity compensation. The sequence of movement, with or without gravity compensation, was randomized across participants to reduce the potential effect of learning or adaptation. Participants had a 15-minute practice period prior to the actual measurements to get accustomed to movements with gravity compensation.

Participants sat in front of a height adjustable table and were secured to the chair with straps to limit compensatory trunk movements. The wrist was fixated by a splint in a position as neutral as possible, midway between flexion/extension and radial/ulnar abduction. Two targets with a diameter of $10 \mathrm{~cm}$ were located on the table surface. The first target was located at a position such that when the hand touched the target, the upper arm was parallel to the trunk and the elbow was flexed at approximately $90^{\circ}$. The second target was $35 \mathrm{~cm}$ from the first target, allowing participants to reach forward in a sagittal plane using both the shoulder and the elbow. Participants started with their hand on the first target and performed repeated multi-joint reach and retrieval movements for 30 seconds, alternating between the 2 targets at a self-selected speed, to match movement paths and movement speeds commonly used in exercises during post-stroke rehabilitation. 


\section{Gravity compensation}

The influence of gravity on the upper extremity was counteracted by a custommade mechanical, passive device (Freebal, see figure 4.1). This device provided a constant amount of gravity compensation throughout the entire workspace of approximately $1 \mathrm{~m}^{3}$ via 2 independent ideal springs, enabling fully compensated 3dimensional arm movements. Compensating forces from the springs, located in the base of the device, are transferred to the arm of the participant by 2 cables running overhead, which are attached to the wrist and elbow by 2 pliable joint braces. By varying the tension of the springs, the amount of gravity compensation is easily adjustable to the arm weight of the participant. A more detailed description of the specifications of Freebal is published elsewhere. ${ }^{17}$

\section{Measurements}

Muscle activity and joint angles of shoulder and elbow were recorded and displayed synchronously to relate muscle activity to movement direction, using software written in Labview (National Instruments, Austin, Texas). Minimum and maximum elbow joint angles (hand on first and second target, respectively), as determined by zero crossings of the elbow angular velocity, defined reversals in

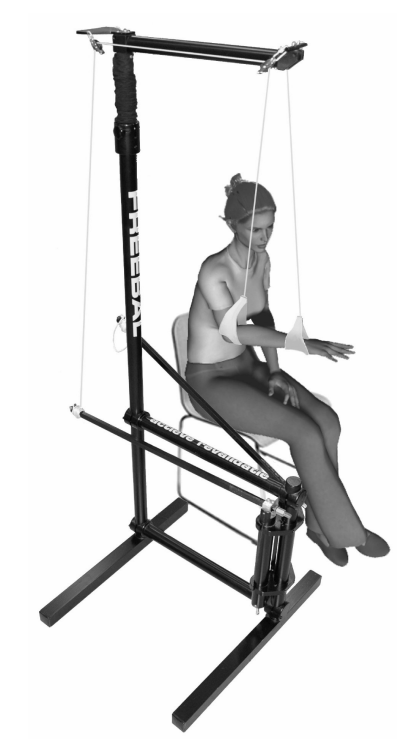

Figure 4.1 Apparatus for gravity compensation: Freebal

Notes: The arm of the participant is attached to the cables of Freebal via a wrist and an elbow strap; the cables are connected to an ideal spring mechanism located in the base of Freebal. 
movement direction. The phase from one maximal elbow extension to the next was defined as a movement cycle, divided in a retrieval (maximal to minimal elbow angle) and reach (minimal to maximal elbow angle) trajectory.

\section{Kinematics}

Arm movements were recorded using an infrared 3D-motion analysis system (VICON; Oxford Metrics Ltd, Oxford, United Kingdom). Joint angles and their definitions were derived from arm segment positions according to guidelines of the International Society of Biomechanics. ${ }^{18}$ The elbow joint angle was specified as the angle between humerus and forearm (elbow extension is defined as $180^{\circ}$ ). Two angles described the shoulder joint orientation. First, the plane of elevation was defined as the angle of the humerus with a virtual line through both shoulders, viewed in the transversal plane (outward/lateral is $0^{\circ}$; arm extended forward is $\left.90^{\circ}\right)$. This represents the angle of the projection of the upper arm on the horizontal plane. Second, the angle of elevation is the angle between humerus and trunk in either a sagittal or a frontal plane, irrespective of the orientation of the humerus in the transversal plane (humerus parallel with trunk is $0^{\circ}$, humerus

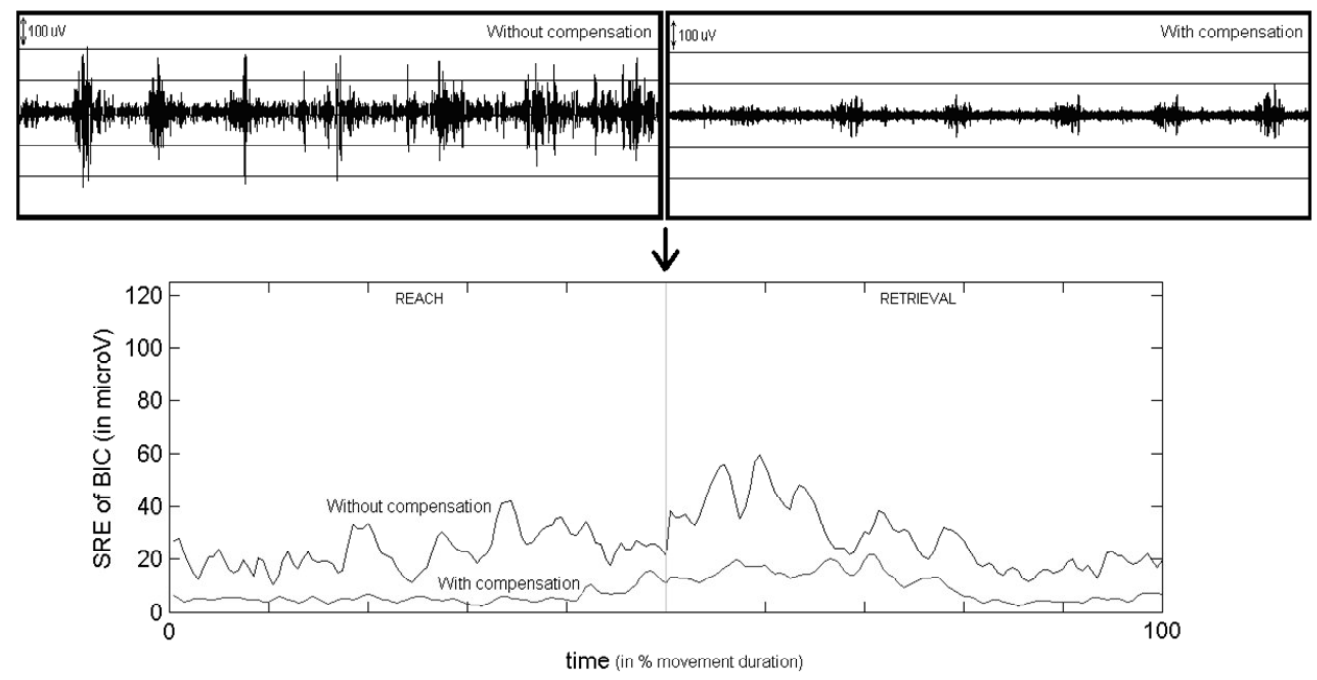

Figure 4.2 EMG recordings of BIC during repeated reach and retrieval

Notes: Filtered, but otherwise unprocessed, electromyographic (EMG) recordings of the long head of biceps (BIC) of a single participant (\#11) during 7 reach and retrieval movements without (top left panel) and with (top right panel) gravity compensation, accompanied by the resulting smooth rectified EMG (SRE) signals of both conditions, averaged over all repeated reach and retrieval movements (bottom panel). 
parallel with horizontal is $90^{\circ}$ ). These angles were low-pass filtered at $20 \mathrm{~Hz}$ with a second order zero-phase shift Butterworth filter and linearly interpolated from 50 to $1000 \mathrm{~Hz}$ to match the sample rate of the electromyography (EMG) recordings.

\section{Muscle activity}

Bipolar surface EMG was recorded from 6 muscles of the upper extremity: biceps, long head (BIC); triceps, long head (TRI); anterior deltoid (DA); middle deltoid (DM); posterior deltoid (DP); and upper trapezius (TRA). EMG was applied using $\mathrm{Ag} / \mathrm{AgCl}$-electrodes (Neuroline, type 720 00-S; Medicotest A/S, Ølstykke, Denmark) and according to the guidelines of the SENIAM project. ${ }^{19}$ The EMG signals were amplified using a K-Lab amplifier (K-Lab, Haarlem, the Netherlands), high-pass filtered (third order Butterworth filter, cut-off frequency $20 \mathrm{~Hz}$ ), and digitized by a 12-bit analog-to-digital converter (integrated in the VICON data station) with a sample rate of 1000 samples per second. These EMG signals were then band-pass filtered (second order zero-phase shift Butterworth, cut-off frequencies $20-400 \mathrm{~Hz}$ ) and converted into smooth rectified EMG (SRE) signals (using a second order zerophase shift Butterworth low-pass filter at $25 \mathrm{~Hz}$ for smoothing) for each muscle per participant. Figure 4.2 shows examples of EMG recordings of BIC of several reach and retrieval movements with and without gravity compensation of a single participant, before and after conversion to SRE signals.

\section{Data analysis}

Parameters of movement performance were determined using the kinematic data. Movement time was defined as the movement cycle duration averaged over all movement cycles per participant (in milliseconds). Movement symmetry was defined as the relative duration of reach with respect to the average movement cycle (in percentages). Joint excursions of elbow and shoulder angles were defined as the difference between minimal and maximal joint angles (in degrees), averaged over all movement cycles.

Averaging the SRE-values and joint angles over the repeated movement cycles, during one 30-second series of reach and retrieval movements, provided an average muscle activation pattern (MAP) for each participant. The first 3 movement cycles were excluded from analysis to take intra-participant and interparticipant differences in movement time into account. The average duration of all repeated movement cycles in 1 series was set at $100 \%$ to take intra-participant and inter-participant differences in movement time into account. The EMG signals and corresponding MAPs of each participant were visually inspected for missing 
data or recording errors. Participants with missing data of elbow joint angles were excluded. Each reach and retrieval trajectory was divided in 3 movement parts: (1) initiation of movement, defined by $0 \%$ to $20 \%$ of a trajectory; (2) forward progression during the middle part of movement, from $30 \%$ to $60 \%$; and (3) termination of movement, consisting of $70 \%$ to $90 \%$ of a trajectory. This subdivision is illustrated in figure 4.3 by means of a typical example. SRE-values were averaged per movement part for each participant as a measure of level of muscle activity. The change of average SRE-values per movement part, across the 3 movement parts, was used as an indication of the general pattern of muscle activity and gross coordination.

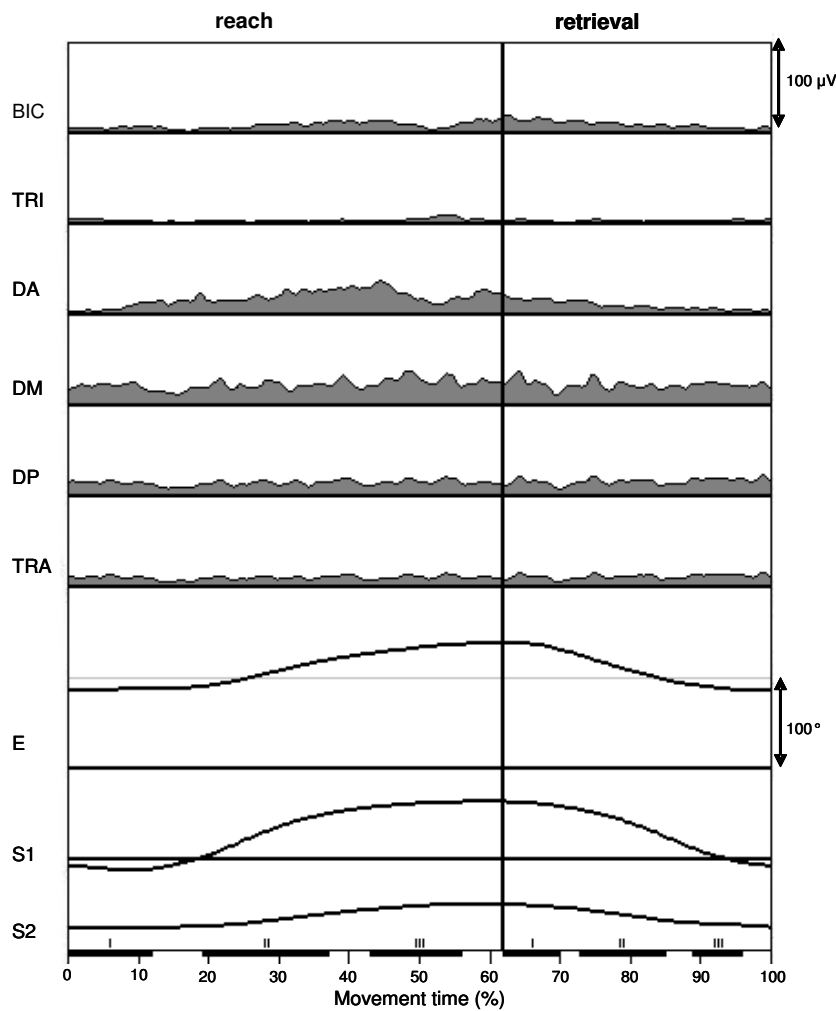

Figure 4.3 Typical example of a muscle activation pattern (MAP)

Notes: Smooth rectified electromyographic (SRE) values of 6 muscles and corresponding joint angles during unsupported movement (time in percentage of averaged cycle duration) of 1 participant are displayed, including definition of movement parts by black bars along the time axis; BIC = biceps, long head; TRI = triceps, long head; $\mathrm{DA}=$ anterior deltoid; $\mathrm{DM}=$ middle deltoid; $\mathrm{DP}=$ posterior deltoid; TRA = upper trapezius; $\mathrm{E}=$ elbow flexion/extension angle; $\mathrm{S} 1$ = shoulder plane of elevation; $\mathrm{S} 2$ = shoulder angle of elevation; I = initiation, II = forward progression, III = termination of movement. 


\section{Statistical analysis}

For statistical analysis, SRE-values were transformed to LN(SRE) values to ensure normal distribution of residuals, as evidenced by a Kolmogorov-Smirnov test of normality ( $p=0.000$ before transformation, $p=0.200$ after transformation) and corresponding normal probability plots.

A linear mixed model was used to test differences in average SRE-values per participant due to gravity compensation (2-level factor "compensation") in each muscle (6-level factor "muscle") per movement part (3-level factor "part"). To account for the correlation of multiple measurements within 1 participant, the intercepts of the model equation were treated as random factors per participant. The factors (compensation, muscle, and part) were treated as fixed effects, since these effects were considered to be similar between participants. The 2-way interactions (muscle $\mathrm{x}$ part, compensation $\mathrm{x}$ muscle, and compensation $\mathrm{x}$ part) were included in the model. For all significant effects and interactions post hoc tests (with Sidak adjustment) were performed.

Furthermore, differences in movement performance parameters between movements with and without gravity compensation were tested using either paired-sample t-tests or Wilcoxon signed ranks tests as a nonparametric equivalent.

To detect potential effects of learning or adaptation, muscle activity and movement performance parameters of the 2 groups performing movements, with and without gravity compensation, in reversed order, were compared using a t-test for independent samples in the case of normally distributed parameters and a nonparametric equivalent, the Kolmogorov-Smirnov test, for parameters deviating from the normal distribution. For all tests the significance level was defined as 0.05 . 


\section{Results}

\section{Participants}

A total of 10 individuals participated in this study, but because of missing data of markers defining the elbow joint angle we had to exclude 2 participants from further analysis. EMG data of BIC of 1 participant was not recorded due to technical problems during data acquisition; however, since the remaining EMG signals and kinematic data were complete, this participant was not excluded. Physical characteristics of the participants are displayed in table 4.1.

Five participants started the experiment with gravity compensation, whereas 3 participants performed first movements without gravity compensation. Comparison of differences in parameters related to gravity compensation revealed no consistent differences in movement performance parameters and EMG parameters between the groups starting the experiment with different conditions of gravity compensation. Therefore, data of all participants were pooled in subsequent analyses.

Table 4.1 Physical characteristics of stroke patients

\begin{tabular}{l|l}
\hline & Subjects $(\mathbf{n = 8})$ \\
\hline Sex (men/women)* & $4 / 4$ \\
Arm dominance (right/left)* $^{*}$ & $6 / 2$ \\
Affected side (right/left)* $^{*}$ & $4 / 4$ \\
Dominant side affected (\%) & 50 \\
Age $(\mathrm{yr})^{\dagger}$ & $63.0( \pm 12.0)$ \\
Height $(\mathrm{m})^{\dagger}$ & $1.75( \pm 0.12)$ \\
Weight $(\mathrm{kg})^{\dagger}$ & $81.4( \pm 24.0)$ \\
BMI $\left(\mathrm{kg} / \mathrm{m}^{2}\right)^{\dagger}$ & $26.3( \pm 4.3)$ \\
Time post-stroke (months) $^{\ddagger}$ & $3.5(1-42)$ \\
FM score (max 66 points) $^{\ddagger}$ & $43.5(33-60)$ \\
\hline
\end{tabular}

Notes: BMI = body mass index; FM = Fugl-Meyer assessment;

* absolute numbers; ${ }^{\dagger}$ mean \pm standard deviation; ${ }^{\ddagger}$ median and range

\section{Movement performance}

A mean $( \pm \mathrm{SD})$ of $12( \pm 4)$ movement cycles per participant were used for analysis. When comparing movement performance, with and without gravity compensation (table 4.2), no differences were observed for movement symmetry and joint excursions of elbow, shoulder plane, and shoulder elevation angles between both 
conditions ( $p=0.590, p=0.386, p=0.626, p=0.499$, respectively). When looking at specific arm orientations only minor differences were observed. Shoulder elevation was somewhat larger at the start of reach with gravity compensation than without gravity compensation ( $59 \pm 4^{\circ}$ and $53 \pm 5^{\circ}$, respectively; $p=0.021$ ). This resulted in a slightly larger extension of the elbow to reach the target with gravity compensation (137 \pm 70 and $132 \pm 8^{\circ}$, respectively; $p=0.001$ ). Besides this, movement times were slightly larger with gravity compensation than during unsupported movements $(p=0.017)$, which was mainly related to a lower movement velocity and not to a larger movement distance. However, the actual difference was very small when movement velocities were calculated $(0.03 \mathrm{~m} / \mathrm{s})$. Therefore, movement performance was regarded to be comparable for both conditions.

Table 4.2 Movement performance parameters

\begin{tabular}{l|ll}
\hline & WITHOUT compensation & WITH compensation \\
\hline Elbow excursion $\left(^{\circ}\right)$ & $44.8( \pm 7.1)$ & $46.5( \pm 10.1)$ \\
Shoulder plane excursion $\left(^{\circ}\right)$ & $52.5( \pm 12.3)$ & $50.0( \pm 21.2)$ \\
Shoulder elevation exc. $\left(^{\circ}\right)$ & $17.1( \pm 5.0)$ & $18.1( \pm 8.9)$ \\
Movement time $(\mathrm{s}){ }^{*}$ & $2.13( \pm 0.67)$ & $2.55( \pm 0.85)$ \\
Movement symmetry $(\%)$ & $58.3( \pm 6.6)$ & $59.3( \pm 6.3)$ \\
\hline
\end{tabular}

Notes: Mean \pm standard deviation is shown from $n=8$, except for shoulder plane excursion, where $n=6$;

* Significant difference between movements with and without gravity compensation $(p<0.05)$.

\section{General aspects of muscle activation}

A typical example of a MAP of movement without gravity compensation is displayed in figure 4.3. In this subject, most muscles displayed activity throughout reach and retrieval, with the exception of TRI, which showed very little or no muscle activity. During reach, a consistent level of muscle activity was observed across movement parts, while during retrieval, muscle activity decreased slightly across movement parts (i.e., from movement initiation to termination) in the majority of muscles.

Several general aspects of muscle activation were identified when MAPs of all participants during movement without gravity compensation (figure 4.4) were compared. All muscles were persistently active without periods of relaxation, except TRI, which had very low levels of activity during both reach and retrieval. The SRE-values changed very little across movement parts during reach $(p=0.547)$, which was consistent for all muscles as supported by a non-significant interaction of "muscle $\times$ part" $(p=0.054)$. On the other hand, SRE-values generally 
decreased across movement parts during retrieval $(p=0.006)$. This decrease was not different between muscles, as shown by a non-significant interaction of "muscle $\times$ part" $(p=0.491)$.

Based on these qualitative and quantitative data, general aspects of muscle activation patterns can be identified for unsupported functional reach and retrieval movements of stroke patients. Muscle activity of BIC lifts and holds the lower arm above the table and aids in anteflexion of the shoulder. TRI is active to contribute to extension of the elbow toward the target, although at a very low level. DA and DM are active to maintain a certain degree of shoulder abduction during movements, to anteflex the shoulder during reach and to decelerate retroflexion of the shoulder during retrieval. Activity of DP decelerates anteflexion of the shoulder during reach and retroflexes the shoulder during retrieval. TRA is active to elevate the upper arm and to position the scapula correctly during reach and retrieval movements.

\section{Influence of gravity compensation}

The comparison of SRE-values between movements with and without gravity compensation (figure 4.5) showed a decrease in the level of muscle activity with gravity compensation during both reach and retrieval $(p=0.000)$, ranging from $25 \%$ to $50 \%$ in most muscles. During reach, the influence of gravity compensation

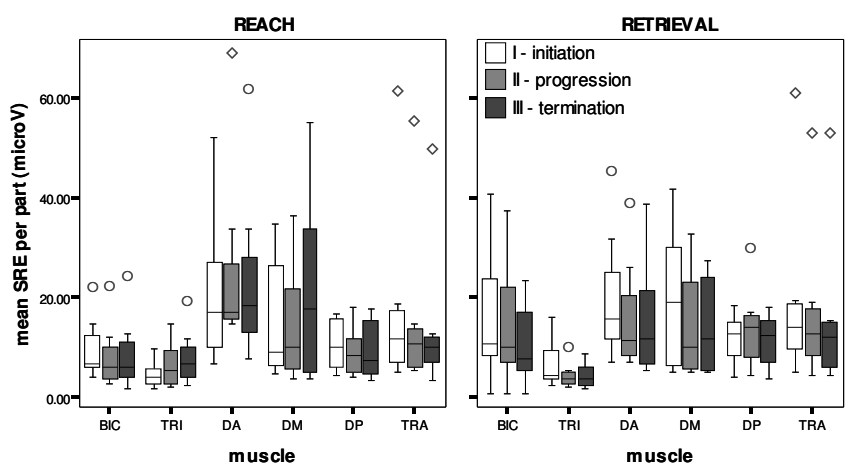

Figure 4.4 Group data of muscle activation patterns (MAPs)

Notes: Median and interquartile ranges of smooth rectified electromyographic (SRE) values of 8 participants per movement part for each muscle during unsupported movement are shown for reach (left panel) and retrieval (right panel); circles represent outliers (deviation of $<1.5$ times interquartile range) and diamonds represent extremes (deviation of $<3$ times interquartile range); BIC $=$ biceps, long head; TRI = triceps, long head; $\mathrm{DA}=$ anterior deltoid; $\mathrm{DM}=$ middle deltoid; $\mathrm{DP}=$ posterior deltoid; TRA = upper trapezius. 
varied between muscles (compensation $\times$ muscle; $p=0.032$ ). SRE-values were lower with gravity compensation in all muscles ( $p \leq 0.001)$, except in TRI (represented in figure 4.5 by asterisks). During retrieval, the influence of gravity compensation was not different between muscles (compensation $\times$ muscle; $p=0.095$ ), meaning that the decrease in SRE-values due to gravity compensation occurred in all muscles.

Regarding the pattern of muscle activity, the change of SRE-values across movement parts was comparable between movements, with and without gravity compensation (figure 4.6), as shown by a non-significant interaction of "compensation $x$ part" in both reach and retrieval $(p=0.485$ and $p=0.956$, respectively). Although the muscle activation pattern of DP during retrieval deviated slightly from this observation, with a larger SRE value during movement termination (movement part III) with gravity compensation than without gravity compensation, this difference was too small to affect the interaction term.

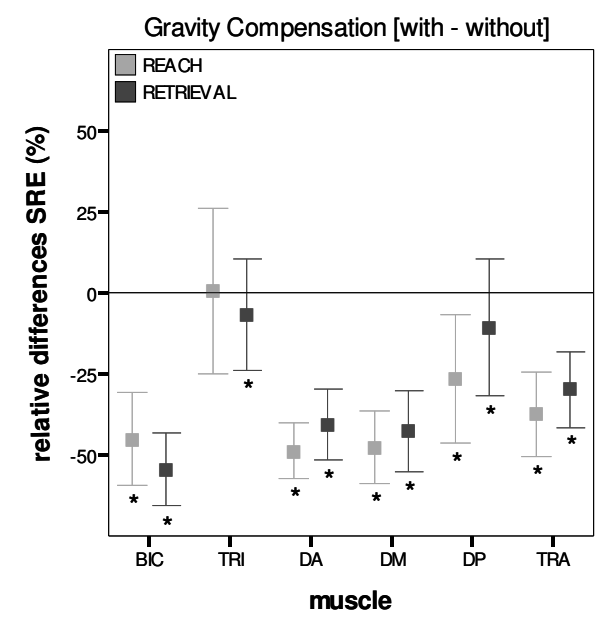

Figure 4.5 Differences in muscle activity due to gravity compensation

Notes: Mean differences ( $\pm 95 \%$ confidence interval) in smooth rectified electromyography (SRE) values, averaged over all movement parts, due to gravity compensation (SRE with gravity compensation - SRE without gravity compensation) are displayed for reach (light symbols) and retrieval (dark symbols) per muscle; asterisks represent significant differences between movements with and without gravity compensation $(p<0.05)$ according to the linear mixed model, taking intra-participant correlation into account; $\mathrm{BIC}=$ biceps, long head; TRI = triceps, long head; $\mathrm{DA}=$ anterior deltoid; $\mathrm{DM}=$ middle deltoid; $\mathrm{DP}=$ posterior deltoid; TRA = upper trapezius. 

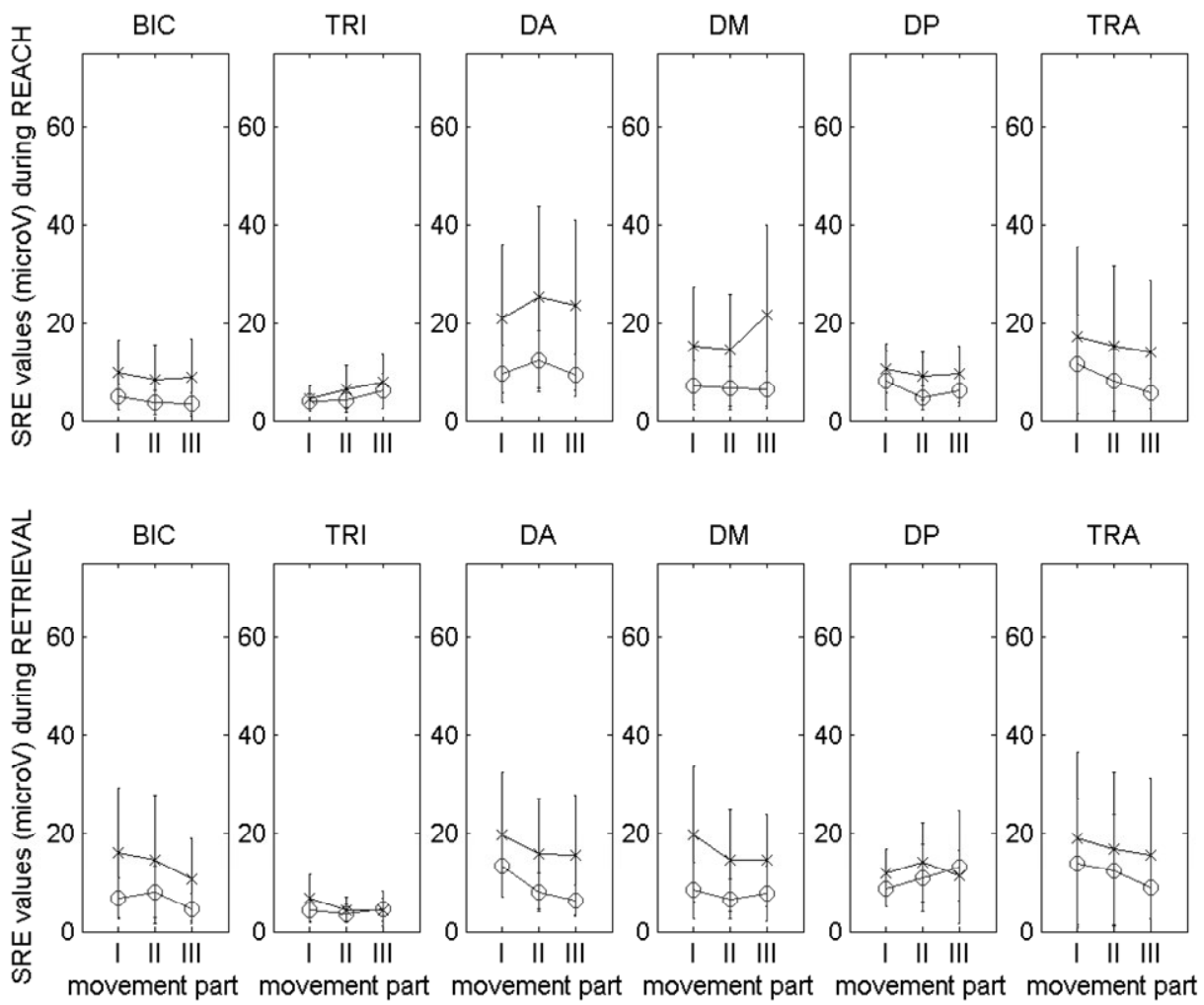

Figure 4.6 Changes in SRE-values across movement parts

Notes: Mean \pm SD smooth rectified electromyography (SRE) values per movement part are shown for movements without (cross markers) and with (circular markers) gravity compensation for each muscle during reach (upper panels) and retrieval (lower panels); BIC = biceps, long head; TRI = triceps, long head; $\mathrm{DA}=$ anterior deltoid; $\mathrm{DM}=$ middle deltoid; $\mathrm{DP}=$ posterior deltoid; $\mathrm{TRA}=$ upper trapezius; I = initiation, II = forward progression, III = termination of movement. 


\section{Discussion}

Our hypothesis was that gravity compensation would influence muscle activation patterns of stroke patients in a positive manner. Knowledge of the nature and direction of these changes will enhance our understanding of underlying working mechanisms of the influence of gravity compensation on improvements in arm movement ability. General aspects of muscle activation during functional reach and retrieval movements, with and without gravity compensation, were identified. Persistent activity was a common aspect during the functional arm movements without gravity compensation in most muscles; there were no periods of complete muscle relaxation. An exception was TRI, which had very low levels of activity throughout the movement. The level of persistent activity did not vary much in muscles from movement initiation to termination during reach, while it decreased somewhat during retrieval. This general pattern was observed in all muscles. A comparison of muscle activation patterns during movements, with and without gravity compensation, showed that the pattern of muscle activity, as represented by the change of average SRE-values across specific aspects of movement, was not influenced by gravity compensation, whereas the level of muscle activity was $25 \%$ to $50 \%$ lower with gravity compensation in all muscles, except in TRI during reach. At the same time, movement time, movement symmetry, and joint angle excursions were comparable in both conditions, although the arm was elevated slightly higher during movements with gravity compensation.

After stroke, muscle activation can be disturbed in many ways with respect to that of healthy persons. Despite this, few differences were observed in the general pattern of muscle activation during these functional reach and retrieval tasks (as indicated by the change of muscle activity level across movement parts) when the MAPs of stroke patients in the present study were compared to those of healthy elderly individuals performing similar movements in a previous study. ${ }^{13}$ It is remarkable that in both stroke patients and healthy elderly persons all muscles, except TRI, were persistently active without periods of muscle relaxation. A plausible explanation for this is that a rather continuous activation is needed to provide stabilization for the many degrees of freedom of the shoulder joint, and to facilitate control of the interaction torques acting on elbow and shoulder joints due to movements of the adjacent joint.

The similarity in MAPs between healthy elderly individuals and stroke patients may be related to the ability of the patients to complete the movement task 
without gravity compensation. The stroke patients included in the present study were mildly to moderately affected, with FM scores ranging between 33 and 60 . The functional reach and retrieval movements were well below the maximal capacity of the stroke patients involved. The nature of these sub-maximal movements contributed to the observed persistent muscle activity, without clear phasic muscle activation, which limited the possibility of a detailed analysis of the timing of muscle activation.

When comparing muscle activity during movements with and without gravity compensation, we found that the level of muscle activity was lower during movements with gravity compensation, while movement performance was comparable. This reduction in level of muscle activity occurred in muscles that act against gravity (BIC, DA, TRA) in stroke patients, as well as in healthy elderly individuals. ${ }^{13}$ As observed in healthy elderly persons, the change of the level of muscle activity across movement parts did not differ between movements with and without gravity compensation. In other words, in both stroke patients and healthy elderly individuals, gravity compensation reduced the level of muscle activity needed to hold the arm in a certain orientation during reach and retrieval, while the general pattern of muscle activation for those movements was comparable. A study by Chabran et al. on segmental postural control in healthy persons reported results along similar lines. ${ }^{20}$ While the presence or absence of an elbow support affects the level of activity of postural muscles (BIC, TRI, DA) during wrist flexion/extension, the timing of activity of the postural muscles is not influenced. ${ }^{20}$ Remarkably, gravity compensation influenced only the level of activity of BIC, DA, and TRA in healthy elderly persons, while in stroke patients the level of activity of DM and DP was also affected, with TRI to a lesser extent. This may be related to an inappropriate coupling of muscles after stroke, ${ }^{15}$ so that gravity compensation affected not only the antigravity muscles directly, but possibly also the coupled, coactivated muscles indirectly.

A limitation of the present study might be that, due to the rather coarse analysis of muscle activity during specific aspects of movement, we could not distinguish whether gravity compensation influenced the specific timing of muscle activation, as indicated by the onset and offset of phasic muscle activity. One could argue that to observe changes in timing, a more demanding task would be more suitable, for instance, by applying ballistic movements or by adding weights to the arm. However, such a fundamental approach was outside the scope of the present study, which focused on functional movements.

In the present study, visual inspection did not reveal any distinct differences in 
timing of muscle activity between movements with and without gravity compensation. Whether changes in timing would occur after an intervention in stroke patients is questionable, given the findings of Chabran et al., who noted no changes in timing of postural muscle contractions with varying elbow support conditions. ${ }^{20}$ In regard to the lower extremity, research by Buurke et al. revealed that timing of muscle activity may not change during recovery of gait in stroke patients, ${ }^{21}$ although timing does differ instantaneously between walking with and without a walking aid. ${ }^{22}$

Regarding movement performance, research indicates a positive influence of gravity compensation. In a previous study, our research group found that the active range of motion increases instantaneously with gravity compensation during multi-joint maximal reaching movements in 3 dimensions with respect to movements without gravity compensation. ${ }^{12}$ Additional analyses showed a simultaneous decrease in the level of muscle activity with gravity compensation. ${ }^{12}$

More fundamental research of Beer and colleagues identified an involuntary coupling between shoulder abduction and elbow flexion in stroke patients, ${ }^{11}$ which is less strong when the arm is supported, resulting in a larger elbow extension during maximal planar reach tasks with arm support. ${ }^{11,23}$ This abnormal coupling probably results from an increased use of alternative neural pathways to compensate for the damaged corticospinal tracts after stroke, which limit the selectivity of muscle activation. ${ }^{24}$

Although gravity compensation did not affect motor control in the current sample of stroke patients as expected, the finding that gravity compensation did reduce the level of activity needed to perform the task indicates that active arm movements may be facilitated during stroke rehabilitation. In this sample of stroke patients with adequate function to complete the task, the facilitation due to gravity compensation occurred without impairing motor control. Due to the support of the arm, the need to generate muscle activity for the postural control of the arm is decreased and patients can use their remaining capacity for generation and coordination of a functional movement, such as reaching for a cup. This facilitating influence of gravity compensation implies that patients may start training of active movements at an early stage and to repeat more movements and/or attend longer or more frequent sessions than in a situation without gravity compensation. This would increase the intensity of therapy to stimulate restoration of arm function during post-stroke rehabilitation. ${ }^{2}$

Gravity compensation is not only applied in conventional rehabilitation, but is also often integrated into new approaches, such as robot-aided therapy, to provide 
arm support during training of arm function. The findings from the present study suggest that the application of gravity compensation alone has the ability to influence the level of muscle activity generated during sub-maximal, functional arm movements, regardless of other potentially integrated training modalities that aim to change arm movement performance during therapy, or a research intervention. This supports the potential of the application of gravity compensation as a separate intervention during post-stroke rehabilitation to stimulate restoration of arm function. 


\section{References}

1. Kwakkel G, Kollen BJ, Van der Grond J, Prevo AJH. Probability of regaining dexterity in the flaccid upper limb: impact of severity of paresis and time since onset in acute stroke. Stroke 2003;34:2181-2186

2. Kwakkel G, Wagenaar RC, Twisk JWR, Lankhorst GJ, Koetsier JC. Intensity of leg and arm training after primary middle-cerebral-artery stroke: a randomised trial. Lancet 1999;354:189-194

3. Feys HM, De Weerdt WJ, Selz BE, et al. Effect of a therapeutic intervention for the hemiplegic upper limb in the acute phase after stroke. A single-blind, randomized, controlled multicenter trial. Stroke 1998;29:785-792

4. Barreca S, Wolf SL, Fasoli S, Bohannon R. Treatment interventions for the paretic upper limb of stroke survivors: a critical review. Neurorehabil Neural Repair 2003;17:220-226

5. Kwakkel G, Kollen B, Lindeman E. Understanding the pattern of functional recovery after stroke: Facts and theories. Restor Neurol Neurosci 2004;22:281-299

6. Cirstea MC, Levin MF. Compensatory strategies for reaching in stroke. Brain 2000;123:940-953

7. Prange GB, Jannink MJA, Groothuis CGM, Hermens HJ, IJzerman MJ. A systematic review of the effect of robot-aided therapy on recovery of the hemiparetic arm after stroke. J Rehabil Res Dev 2006;43(2):171-184

8. Wagenaar RC, Meijer OG. Effects of stroke rehabilitation: a critical review of the literature. J Rehabil Sci 1991;4(3):61-73

9. Johnson MJ. Recent trends in robot-assisted therapy environments to improve reallife functional performance after stroke. J Neuroengineering Rehabil 2006;3:29

10. Amirabdollahian F, Loureiro R, Gradwell E, Collin C, Harwin W, Johnson G. Multivariate analysis of the Fugl-Meyer outcome measures assessing the effectiveness of GENTLE/S robot-mediated stroke therapy. J Neuroengineering Rehabil 2007;4(4)

11. Beer RF, Dewald JPA, Dawson ML, Rymer WZ. Target-dependent differences between free and constrained arm movements in chronic hemiparesis. Exp Brain Res 2004; 156:458-470

12. Prange GB, Stienen AHA, Jannink MJA, Van der Kooij $H$, IJzerman MJ, and Hermens $\mathrm{HJ}$. Increased range of motion and decreased muscle activity during maximal reach with gravity compensation in stroke patients. In: Proceedings of the 10th International Conference on Rehabilitation Robotics (ICORR); June 13-15, 2007, Noordwijk aan Zee, the Netherlands:467-471

13. Prange GB, Kallenberg LAC, Jannink MJA, et al. Influence of gravity compensation on muscle activity during reach and retrieval in healthy elderly. J Electromyogr Kinesiol 2009;19:e40-e49

14. Papaxanthis C, Pozzo T, Popocv KE, McIntyre J. Hand trajectories of vertical arm 
movements in one-G and zero-G environments. Evidence for a central representation of gravitational force. Exp Brain Res 1998;120(4):496-502

15. Chae J, Yang G, Park BK, Labatia I. Muscle weakness and cocontraction in upper limb hemiparesis: relationship to motor impairment and physical disability. Neurorehabil Neural Repair 2002;16:241-248

16. Fugl-Meyer AR, Jääskö L, Leyman I, Olsson S, Steglind S. The post-stroke hemiplegic patient. A method for evaluation of physical performance. Scand J Rehab Med $1975 ; 7: 13-31$

17. Stienen AHA, Hekman EEG, Van der Helm FCT, Prange GB, Jannink MJA, Aalsma AMM, and Van der Kooij H. Freebal: dedicated gravity compensation for het upper extremities. In: Proceedings of the 10th International Conference on Rehabilitation Robotics (ICORR); June 13-15, 2007, Noordwijk aan Zee, the Netherlands:804-808

18. Wu G, Van der Helm FC, Veeger HE, et al. ISB recommendation on definitions of joint coordinate systems of various joints for the reporting of human joint motion Part II: shoulder, elbow, wrist and hand. J Biomech 2005;38(5):981-992

19. Hermens $\mathrm{HJ}$, Freriks $B$, Merletti $\mathrm{R}$, et al. European recommendations for surface electromyography, results of the SENIAM project. Enschede: Roessingh Research and Development; 1999

20. Chabran E, Maton B, Ribreau C, Fourmant A. Electromyographic and biomechanical characteristics of segmental postural adjustments associated with voluntary wrist movements. Influence of an elbow support. Exp Brain Res 2001;141:133-145

21. Buurke JH. Walking After Stroke. Co-Ordination Patterns and Functional Recovery (Dissertation). Enschede: University of Twente, Enschede, the Netherlands; 2005

22. Buurke $\mathrm{JH}$, Hermens $\mathrm{HJ}$, Erren-Wolters $\mathrm{CV}$, Nene AV. The effect of walking aids on muscle activation patterns during walking in stroke patients. Gait Posture 2005;22(2):164-170

23. Beer RF, Given JD, Dewald JPA. Task-dependent weakness at the elbow in patients with hemiparesis. Arch Phys Med Rehabil 1999;80:766-772

24. Beer RF, Ellis MD, Holubar BG, Dewald JPA. Impact of gravity loading on post-stroke reaching and its relationship to weakness. Muscle Nerve 2007;36:242-250 



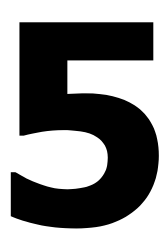

\title{
AN EXPLORATIVE, CROSS-SECTIONAL STUDY INTO ABNORMAL MUSCLE SYNERGIES DURING FUNCTIONAL REACH IN CHRONIC STROKE PATIENTS
}

\author{
GB Prange \\ MJA Jannink \\ AHA Stienen \\ $\mathrm{H}$ van der Kooij \\ MJ IJzerman \\ HJ Hermens
}

submitted 2009 


\section{Abstract}

Introduction - In many stroke patients, arm function is limited, which can be related to a reduced selectivity of movement due to abnormal coupling between different joints. The extent of abnormal coupling between shoulder and elbow movements in chronic stroke patients in terms of muscle activation during functional movements is rather unknown. Therefore, the present study compared synergistic patterns in muscle activation during functional reaching movements between chronic stroke patients and healthy persons.

Methods - To provoke synergistic patterns during movement, upward reaching movements were performed with and without resistance against shoulder elevation. Differences in movement performance (joint angles) and muscle activation (amplitude of activity) due to shoulder elevation resistance were compared between healthy persons and stroke patients using a repeated measures ANOVA.

Results - Changes in movement performance and muscle activation during upward reaching due to shoulder elevation resistance were not significantly different between chronic stroke patients and healthy persons.

Conclusions - These findings suggest that tasks corresponding with daily activities, without near-maximal effort, do not elicit abnormal coupling between shoulder and elbow movements in mildly affected chronic stroke patients. This implies that for this group of patients it would not be necessary to focus rehabilitation on reducing abnormal synergies, but can be aimed at other impairments that may be more involved in limiting arm function. 


\section{Introduction}

After stroke, limitations in arm function are common. ${ }^{1}$ Varying sensory and motor symptoms contribute to a reduced ability to coordinate movements. ${ }^{2}$ This can, amongst others, be expressed as an involuntary coupling of movements, as was already recognized by Brunnstrom several decades ago. ${ }^{3}$ She distinguished two patterns of coupling to describe the motor behavior of stroke patients: a flexion synergy and an extension synergy. For the upper extremity, the flexion synergy pattern includes shoulder abduction, shoulder external rotation, elbow flexion and forearm supination, while the extension synergy pattern comprises shoulder adduction, shoulder internal rotation, elbow extension and forearm pronation.

Beer, Dewald and colleagues showed that in isometric contractions of chronic stroke patients the generation of shoulder abduction torques is coupled to simultaneous generation of elbow flexion torques: the higher the shoulder abduction torque, the more elbow flexion is generated. ${ }^{4,5}$ When extending this research to dynamic situations, they found limitations in elbow extension during reaching without arm support when the arm has to be lifted actively, since active shoulder abduction provoked simultaneous elbow flexion torques. ${ }^{6}$

Besides this insight into kine(ma)tics of abnormal synergies in stroke, only some information is available about muscle activity during these abnormal synergies. Dewald et al. indicated that activity of the shoulder abducting muscles, deltoid and upper trapezius, is correlated to elbow flexor muscles and that the shoulder adducting pectoral muscle is activated concurrently with elbow extensor muscles during isometric torque generation with the affected arm of chronic stroke patients at shoulder height. ${ }^{4}$ These findings indicate that the flexion and extension synergies are also expressed in muscle activity during simultaneous isometric contractions of shoulder and elbow muscles after stroke. However, it is not known whether these findings can be extended to a dynamic situation (actual reaching movements) corresponding with functional movements as applied in everyday life.

Information about muscle activation of abnormal synergies during functional reaching movements will provide insight into the neurophysiology underlying potential torque-couplings between the shoulder and elbow during activities of daily living. This will provide starting points for the design of specific rehabilitation programs to stimulate more selective control of shoulder and elbow movements. Such focused approaches may in turn improve outcome of rehabilitation aiming at restoration of arm function. 
This study was designed to examine whether abnormal synergistic patterns are expressed in muscle activation during sub-maximal functional multi-joint reaching movements of chronic stroke patients. For this purpose, upward reaching movements were performed with and without resistance against arm elevation. To identify abnormal patterns of coupling, movements of chronic stroke patients were compared to those of healthy persons, with the expectation that in chronic stroke patients an increased generation of shoulder elevation torques is accompanied by a reduction in elbow extension during reaching movements with shoulder elevation resistance and an increased coupling of shoulder abductor and elbow flexor muscles. 


\section{Methods}

\section{Subjects}

A random sample of 15 chronic stroke patients, receiving or having received care from a local rehabilitation centre, was selected. For participation in the study subjects had to meet the following inclusion criteria: (1) age between 25 and 75 years; (2) at least 6 months post-stroke; (3) ability to lift the arm (at least partly) against gravity, without full recovery of selective shoulder and elbow movements; (4) no pain or other condition interfering with the mobility and/or strength of the arm; (5) ability to understand and follow instructions; (6) provide written informed consent.

Five healthy persons with no history of arm function impairments were included to compare findings in chronic stroke patients with unimpaired movement control and performance. The study was approved by the local medical ethics committee (METC of Rehabilitation Center Het Roessingh, Enschede, the Netherlands).

\section{Procedure}

During the reaching task, subjects were seated with straps over the trunk to limit compensational trunk movements, with the upper arm aligned with the trunk (shoulder in $0^{\circ}$ anteflexion and $0^{\circ}$ abduction) and the elbow flexed $90^{\circ}$ (figure 5.1). The wrist was placed in a position as neutral as possible by fixation in a splint (midway between flexion/extension and radial/ulnar abduction) and the hand was balled to a fist as much as possible. A starting square of $10 \times 10 \mathrm{~cm}$ was placed under the subject's hand and the target square of $10 \times 10 \mathrm{~cm}$ was placed just below shoulder height at $90 \%$ of the subject's active range of motion and approximately $30^{\circ}$ lateral from the shoulder, so that upward and outward reaching movements

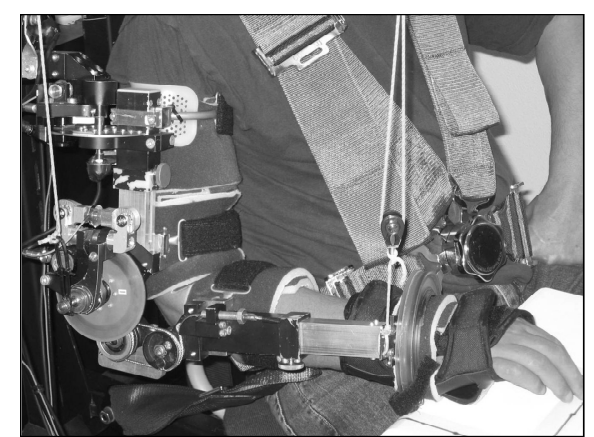

Figure 5.1 Dampace exoskeleton for joint-specific resistance 
were required using both shoulder and elbow rotations.

During repetitive multi-joint reaching movements, arm elevation was resisted by $80 \%$ of the shoulder elevation torque needed to lift the arm. In healthy persons, this corresponded with $11-19 \%$ of maximal voluntary shoulder elevation torque generation, in stroke patients this ranged from $23-65 \%$ of maximal voluntary shoulder elevation torque generation. Reaching movements were repeated 20 times in both conditions (with and without shoulder elevation (SE) resistance). Prior to each measurement subjects got accustomed to reaching with the exoskeleton with the corresponding resistance by repeating the required movement several times. The reaching movements were performed at a selfselected speed.

\section{Application of resistance}

In order to provoke or reinforce a flexion synergy during functional reaching movements, the required torque to elevate the arm was increased by applying joint-specific resistance during reach with the use of a newly developed robotic device (Dampace, see figure 5.1). ${ }^{7}$ The Dampace device is an exoskeleton structure, attached to the upper and lower arm by soft straps, which can apply resistance to each of the shoulder and elbow axes. Three degrees of freedom at the shoulder enable transversal rotation (corresponding with horizontal abduction), elevation (which corresponds with shoulder abduction and/or flexion expressed within the clinical framework), and axial rotation (corresponding with endo/exorotation) of the upper arm. One degree of freedom at the elbow enables flexion/extension and a flexible wrist attachment allows pro-/supination of the forearm. The exoskeleton is attached to a rigid frame, situated behind the subject, in such a way that the shoulder and scapula can move freely. More details of the Dampace can be found in Stienen et al. 2007. ${ }^{7}$

Specific resistance torques were applied to the shoulder elevation axis by a hydraulic disc brake. The braking force is controlled by a computer, based on measurements of integrated position and torque sensors. Besides this, the gravitational pull on the exoskeleton and the arm was compensated by a system of ideal springs, attached to the exoskeleton by wires via several pulleys overhead.

\section{Measurements}

At the start of the experiment, the upper extremity portion of the Fugl-Meyer assessment (FM) was performed to document the status of motor recovery and arm function of the hemiparetic arm. 


\section{Muscle activity}

Bi-polar surface electromyography (EMG) was recorded from 8 upper extremity muscles using Ag/AgCl-electrodes (Neuroline, type 720 00-S; Medicotest A/S, Ølstykke, Denmark), according to the guidelines of the SENIAM project. ${ }^{8}$ The EMG signals of biceps (BIC), brachioradialis (BRA), long and lateral head of triceps (TILO and TILA), anterior deltoid (AD), posterior deltoid (PD), lattissimus dorsi (LD) and upper trapezius (TRA) were measured and amplified using a 16-channel Porti system (Twente Medical Systems International, Oldenzaal, the Netherlands) and digitized by a 22-bit analog-to-digital converter with a sample rate of 1024 samples per second and stored on a computer. For real-time display, the EMG signals were high-pass filtered (3rd order Butterworth filter, cut-off frequency 5 $\mathrm{Hz}$ ) during the measurements. The recorded EMG signals were, off-line, band-pass filtered (2nd order zero phase shift Butterworth, cut-off frequencies $10-400 \mathrm{~Hz}$ ) and converted to smooth rectified EMG (SRE) signals (using a low-pass 2nd order zero phase shift Butterworth filter at $25 \mathrm{~Hz}$ for smoothing).

\section{Kinematics}

Kinematic data of arm segments were recorded using integrated position sensors in the Dampace at each movement axis of the shoulder and elbow. The voltages over the potentiometers at the three shoulder axes were converted from analog to digital values by a DAQcard (National Instruments, Austin, Texas) with a sample rate of $1000 \mathrm{~Hz}$. The elbow angle is measured by an integrated two-channel rotational optical encoder (US Digital, Vancouver, Washington). The elbow joint angle was specified as the angle between humerus and forearm (maximal elbow extension is $180^{\circ}$ ). The shoulder joint orientation was described using two angles according to recommendations of the International Society of Biomechanics. ${ }^{9}$ The plane of elevation (transversal rotation or horizontal abduction) was defined as the angle of the humerus with a virtual line through both shoulders, viewed in the transversal plane (outward/lateral is $0^{\circ}$, arm extended forward is $90^{\circ}$ ). The angle of elevation (shoulder abduction and/or flexion) was the angle between humerus and trunk in the plane of elevation (consisting of the vertical plane through the upper arm), irrespective of the orientation of the humerus in the transversal plane (humerus parallel with trunk is $0^{\circ}$, humerus horizontal is $90^{\circ}$ ). These data were real-time filtered with a first order Butterworth low-pass filter with a cut-off frequency of $40 \mathrm{~Hz}$. Filtering is performed in a Simulink model (The Mathworks Inc, Natick, Massachusetts) which is compiled into an executable using the RealTime Application Interface for Linux (www.rtai.org). Measured signals are stored on a 
computer with a sample frequency of $50 \mathrm{~Hz}$. Off-line, the kinematic data were linearly interpolated from 50 to $1024 \mathrm{~Hz}$ to match the sample rate of the EMG recordings.

\section{Data analysis}

The SRE-signals and joint angles were synchronized and averaged over all repeated reaching movements within one phase, for each muscle and per subject. Reaching movements were defined by the elbow joint angle, with the minimum angle representing the start of reach and the maximum angle representing the end of reach. The duration of the reaching movement was expressed as $100 \%$, to account for intra- and inter-subject variation.

Analysis comprised initial qualitative inspection of muscle activation patterns and subsequent calculation of quantitative measures. The level of muscle activity was represented by the mean SRE-value during the averaged reaching movement per resistance phase. To evaluate relative changes in the contribution of each muscle to reach within each subject, the SRE-value of each muscle was related to the total amount of SRE generated by all 8 muscles combined during the reaching movement of one subject (input\%; percentage of mean SRE-value of each muscle with respect to the cumulative SRE-value of all 8 muscles per subject). Additional information about inter-muscle coupling in each subject was provided by individually calculating the ratio between the average SRE-values of elbow flexors (BIC and BRA) and the prime mover for the shoulder during the current movement task (AD) so that co-contraction ratios (CCratios) of BIC and AD and of BRA and $A D$ were obtained.

To quantify movement performance, movement time (in ms), minimal (i.e., at the start of reach), maximal (i.e., at the end of reach) angles (in ${ }^{\circ}$ ) of shoulder and elbow joints and the difference between minimal and maximal joint angles (i.e., joint excursion or range of motion) were calculated for each averaged reaching movement. All changes in outcome measures between reaching movements with and without shoulder elevation resistance (SE-resistance) were compared between healthy subjects and chronic stroke patients.

\section{Statistical analysis}

All outcome measures were inspected for normal distribution of data using histogram plots including normal curves and normal probability plots prior to selection of proper statistical tests. Differences in movement time between movements with and without SE-resistance in both healthy persons and chronic 
stroke patients were tested using a paired samples t-test, or its non-parametric equivalent (Wilcoxon signed ranks test). Minimal, maximal and range of motion (ROM) values of all joint angles were compared between movements with and without SE-resistance (within-subjects factors of 'resistance', 'joint' and 'gonio') and between healthy persons and chronic stroke patients (between-subjects factor of 'status') using analysis of variance (ANOVA) for repeated measures.

SRE-values and CCratios were log-transformed prior to statistical analysis to ensure normal distribution of the data. For each muscle, mean SRE-values and input\% were compared between movements with and without SE-resistance and between healthy persons and chronic stroke patients using an ANOVA for repeated measures, using a within-subjects factor for 'resistance' (with or without SEresistance) and a between-subjects factor for 'status' (healthy or stroke). The same procedure was repeated for the two CCratios. For all tests, the significance level was defined as 0.05 . 


\section{Results}

\section{Subjects}

One of the 15 included stroke patients was not able to complete the tasks due to severe limitations in arm function. Of another stoke patient the data was not complete due to technical problems during measurement. The data of these 2 subjects were excluded from data analysis. Data of 5 healthy persons (4 male) and 13 stroke patients ( 9 male) was available for analysis (see table 5.1 for details). All stroke patients were in the chronic phase, with the time post-stroke varying from 7 to 126 months. The level of arm function, as measured by the FM assessment, ranged from 22 to 65 , with an average score of 51 points. Of the 13 stroke patients, 9 had FM scores larger than 45 points, whereas 4 patients had FM scores lower than 45 points, indicating that the majority of stroke patients in the present study had mild hemiparesis.

Table 5.1 Descriptive (mean \pm SD) subject characteristics

\begin{tabular}{l|ll}
\hline & Healthy subjects $(\mathbf{n = 5})$ & Stroke patients $(\mathbf{n = 1 3})$ \\
\hline Age (years) & $54.4( \pm 19.0)$ & $65.9( \pm 6.9)$ \\
Body mass index $\left(\mathrm{kg} / \mathrm{m}^{2}\right)$ & $22.5( \pm 0.99)$ & $25.5( \pm 3.6)$ \\
Time post-stroke (months) & not applicable & $26( \pm 31)$ \\
FM score (points of max 66) & not applicable & $51( \pm 13)$ \\
\hline
\end{tabular}

\section{Movement performance}

Mean movement time did not differ significantly between movements with and without SE-resistance in healthy subjects and chronic stroke patients $(p \geq 0.510)$. When comparing both groups, chronic stroke patients showed somewhat larger movement times than healthy persons (respectively $1.3 \mathrm{~s}$ and $0.9 \mathrm{~s}, \mathrm{p} \leq 0.034$ ).

Mean joint angle extremes and ranges of healthy persons and stroke patients are displayed in figure 5.2 per resistance condition. Inspection of these data showed that in both groups angles and excursions of several joints decreased with resistance. Maximal elbow (E) and shoulder elevation (SE) angles (at the end of reach) and their excursions (ROM) were $7^{\circ}$ to $14^{\circ}$ smaller with resistance ('resistance' $p \leq 0.004$ ). Although this led to SE angles smaller than $5^{\circ}$ in 5 of the 13 stroke patients, all subjects (both healthy persons and stroke patients) could still reach the target at shoulder height with resistance. Minimal angles (at the start of reach) were similar in both conditions, except for the minimal E-angle, which was 
slightly smaller (i.e., the elbow was more flexed) with an average of $3^{\circ}$ at the start of resisted reach $(p=0.015)$. The shoulder plane of elevation (SP) remained largely unchanged. Despite these changes within subjects, no significant differences were found between healthy persons and chronic stroke patients.

\section{Muscle activation}

To examine the expression of any abnormal coupling between muscles in chronic stroke patients, we investigated changes in muscle activity due to the application of SE-resistance.

\section{Muscle activity levels}

With respect to movements without SE-resistance, the activity of all muscles increased during movements with SE-resistance, in both healthy persons and chronic stroke patients (figure 5.3). The increases of $A D, T R A$ and to a smaller extent BIC reflect the enhanced SE-torque to be generated with resistance. This slightly increased BIC activity requires some increase in activity of the elbow extensor muscles (TILO and TILA) to achieve the reaching task. In addition, it is likely that with SE-resistance more stabilization of the shoulder joint is needed to control the larger generated shoulder elevation forces, resulting in slightly increased activity levels of PD and LD. These increases were significant in all muscles ('resistance' $p \leq 0.007$ ).

When comparing healthy persons and chronic stroke patients, few differences in SRE-values were found between both groups ('status' $p \geq 0.176$ ). One of the
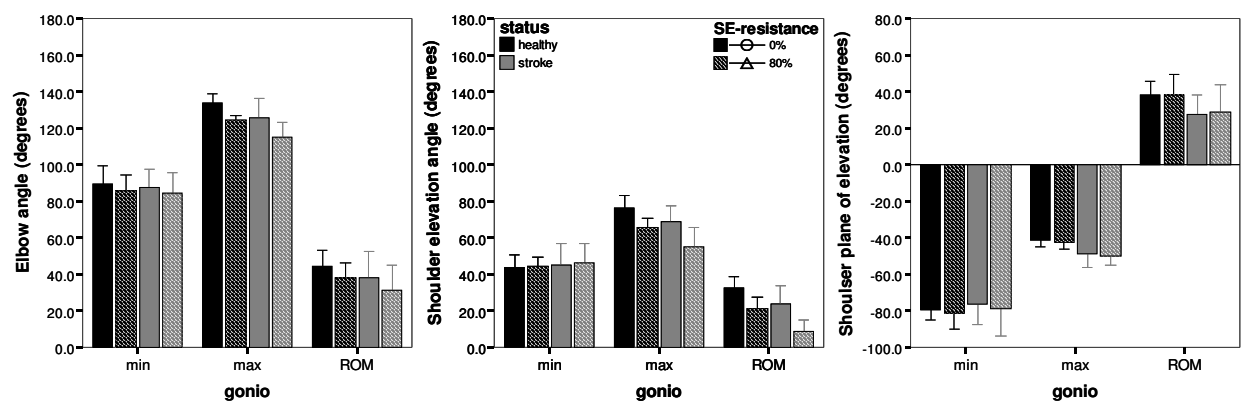

Figure 5.2 Mean $( \pm S D)$ of extremes and ranges of joint angles with and without shoulder elevation (SE) resistance

Notes: Different panels display elbow flexion/extension (A), shoulder angle of elevation (B), and shoulder plane of elevation $(C)$ in healthy persons (black bars) and chronic stroke patients (grey bars) per resistance condition ( $0 \%$ and $80 \%$ in solid and striped bars, resp.). 
differences concerns TILA, in which overall SRE-values were higher in chronic stroke patients compared to healthy persons $(p=0.019)$. The increases in SREvalues with SE-resistance also did not differ significantly between healthy persons and chronic stroke patients in most muscles ('status $x$ resistance' $p \geq 0.082$ ), except for TILO $(p=0.019)$ and PD $(p=0.009)$. In those two muscles, the increases in SREvalues with resistance were smaller in chronic stroke patients than in healthy persons.

Since the increases in SRE-values with resistance are not more pronounced in chronic stroke patients than in healthy persons, especially regarding shoulder elevators and elbow flexors, these findings do not point to an enhanced expression of abnormal synergies in chronic stroke patients during functional movements.

\section{Contribution of individual muscles to reach}

When looking at the contribution of each muscle to reach within each subject (input\%), it is observed that the application of SE-resistance hardly changed the distribution of input\% between muscles (figure 5.4). Only input\% of BRA decreased somewhat when SE-resistance was applied ('resistance' $p=0.014$ ), over all subjects.

Few differences were found between healthy persons and chronic stroke patients. The input\% of AD was smaller and the input\% of TILA was larger in

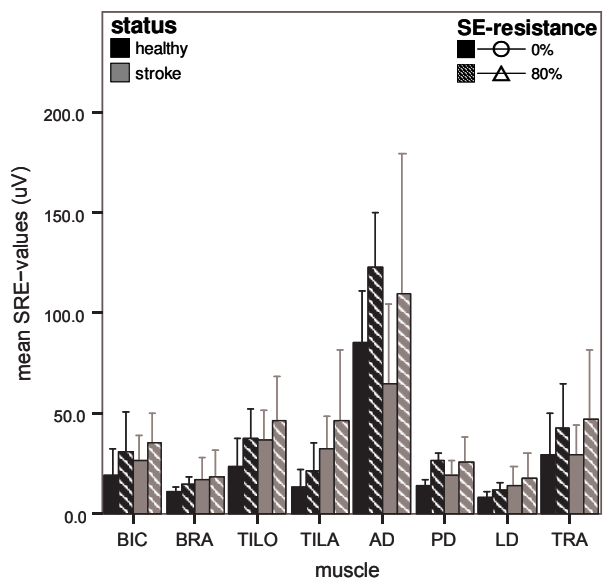

Figure 5.3 Mean ( $\pm S D$ ) smooth rectified EMG (SRE) values per muscle with and without shoulder elevation (SE) resistance

Notes: Data of healthy persons are displayed in black bars and of chronic stroke patients in grey bars per resistance condition ( $0 \%$ and $80 \%$ in solid and striped bars, resp.). 
chronic stroke patients than in healthy persons ('status' $p \leq 0.034$ ). Changes in input\% with SE-resistance only differed slightly between healthy persons and chronic stroke patients in TILO and PD. With SE-resistance, input\% of TILO decreased in chronic stroke patients, whereas it did not change in healthy persons ('status $x$ resistance' $p=0.032$ ). In $P D$, input\% increased with SE-resistance in healthy persons, whereas no significant change was detected in chronic stroke patients ('status $\mathrm{x}$ resistance' $\mathrm{p}=0.011$ ).

Although some small differences in muscle contributions were found between healthy persons and chronic stroke patients, these differences were not consistent with an increased coupling between S-abductors and E-flexors.

\section{Co-contraction of shoulder and elbow muscles}

Additional information about specific inter-muscle coupling within each subject was obtained by relating the individual average SRE-values of elbow flexors (BIC and $B R A$ ) to the prime mover for the shoulder during the reaching task (AD).

When comparing the values of the mean CCratio of BIC and AD between healthy persons and chronic stroke patients (figure 5.5), we found that the CCratio remained largely unchanged with resistance ('resistance' $p=0.557$ ) in both healthy persons and chronic stroke patients ('status $x$ resistance' $p=0.379$ ). Overall, the

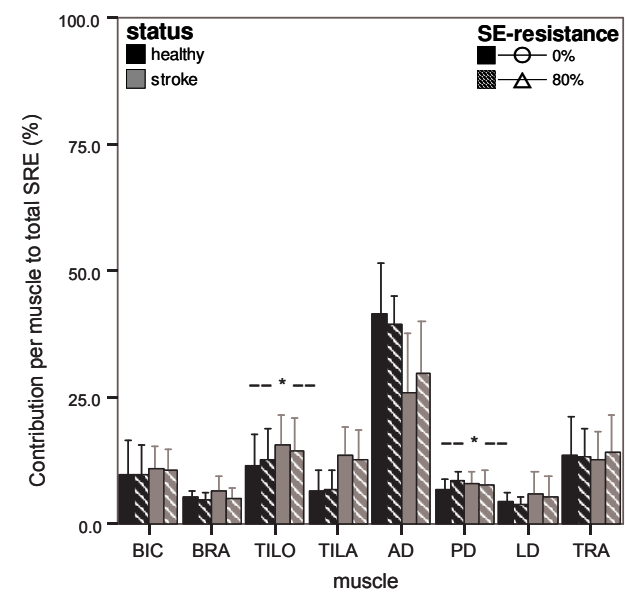

Figure 5.4 Mean $( \pm S D$ ) of relative muscle contributions with and without shoulder elevation (SE) resistance

Notes: Data of healthy persons are displayed in black bars and of chronic stroke patients in grey bars per resistance condition ( $0 \%$ and $80 \%$ in solid and striped bars, resp.); asterisks indicate significant differences in changes due to SE-resistance between healthy persons and chronic stroke patients. 
CCratio did also not differ significantly between healthy persons and chronic stroke patients ('status' $\mathrm{p}=0.091$ ).

The differences in co-contraction of BRA and AD (figure 5.5) with SE-resistance and between groups are comparable to those of BIC and AD. Statistically, differences were slightly more pronounced, due to a smaller variation in CCratio for BRA and AD across subjects. With SE-resistance, the CCratio decreased significantly when looking overall over both groups ('resistance' $p=0.011$ ). When comparing the CCratios of BRA and $A D$ for healthy persons and chronic stroke patients, no significant differences were observed ('status' $p=0.114$ ). Also, the decreases with resistance were not significantly different between healthy persons and chronic stroke patients ('status $x$ resistance' $p=0.094$ ).

Again, these results do not point to an abnormal coupling between $A D$ and elbow flexors, since an increase in $A D$ activity was accompanied by a less than proportional increase in BIC and BRA activity in both healthy persons and chronic stroke patients.

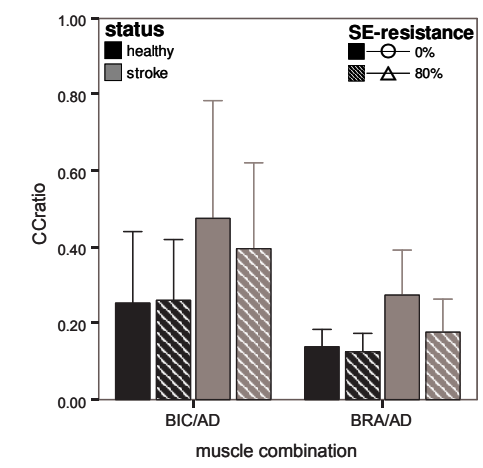

Figure 5.5 Mean ( $\pm S D$ ) of co-contraction ratios (CCratios) of shoulder elevators (AD) with elbow flexors (BIC and BRA)

Notes: Data of ratios of biceps (BIC) to anterior deltoid (AD) and brachioradialis (BRA) to AD are displayed for healthy persons (black bars) and chronic stroke patients (grey bars) per resistance condition ( $0 \%$ and $80 \%$ in solid and striped bars, resp.). 


\section{Discussion}

To examine whether an abnormal coupling of shoulder elevation and elbow flexion is expressed in muscle activation during functional reaching movements of chronic stroke patients, the present study compared changes in muscle activation between sub-maximal, three-dimensional reaches with and without shoulder elevation resistance at a comfortable speed between healthy persons and chronic stroke patients. The term shoulder elevation as defined in the present study corresponds with shoulder abduction and/or flexion as commonly used in clinical practice.

The present study did not find significant differences in changes in joint angles and joint excursions due to shoulder elevation resistance in chronic stroke patients in comparison with healthy persons, although movement speed was somewhat lower. All subjects, both healthy persons and stroke patients, were able to reach the target both without and with SE-resistance. However, in 5 out of 13 stroke patients shoulder elevation excursion was reduced to less than $5^{\circ}$ with resistance, making it more difficult to detect a potential abnormal flexion synergy if it were present. Nonetheless, the chronic stroke patients in the present study retained the ability to slightly adjust their movement to restrictions in the environment, so that the desired movement task could still be accomplished. This suggests that in none of the subjects an abnormal flexion synergy restricted movement in such a way that they could not reach the target.

Concerning muscle activation, the increases in level of muscle activation with shoulder elevation resistance, observed in all muscles, were comparable between healthy persons and chronic stroke patients. In addition to the absence of a substantial reduction of elbow extension during resisted reach in chronic stroke patients, no indications were found that during reach with resistance an increase in $A D$ activity was accompanied by a larger increase in elbow flexor activity in chronic stroke patients compared to healthy persons, when looking at the relative contribution of each muscle (input\%)and co-contraction ratios. These findings do not support the occurrence of abnormal coupling between shoulder abductors and elbow flexors during functional reaching movements by mildly affected chronic stroke patients in the present study.

Conversely, Beer and colleagues found a coupling of shoulder elevation torques to simultaneous generation of elbow flexion torques in chronic stroke patients, resulting in reduced elbow extension ability in both static and dynamic situations. 5 Dewald et al. showed that activity of shoulder abducting muscles is correlated with 
activity of elbow flexor muscles during isometric torque generation in chronic stroke patients. ${ }^{4}$ It is possible that part of this discrepancy is related to differences in stroke severity of the participants in both studies. The chronic stroke patients included in the present study varied in severity of hemiparesis from patients who could just lift their own arm (FM score of 22) to patients who experienced very few limitations in arm function (FM score of 65), although the majority of stroke patients displayed mild hemiparesis. The research by Beer, Dewald and colleagues involved chronic stroke patients with a more severe arm paresis; FM scores ranged from 15 to 60 points in initial research, ${ }^{4}$ and were even lower in later work with FM scores ranging from 15 to 40 points. ${ }^{10}$ In more severely affected patients abnormal coupling may play a more pronounced role than in patients with less limitation in arm function.

Differences concerning the arm position during dynamic evaluations of abnormal coupling are also a plausible cause for the discrepancy. In the research of Beer, Dewald and colleagues, dynamic tasks required an arm position of $75^{\circ}$ up to $90^{\circ}$ of shoulder abduction during the entire movement task. ${ }^{6,11-13}$ The occurrence of the coupling between shoulder abduction and elbow flexion was found to be dependent on the magnitude of generated torques, ${ }^{5}$ indicating that in the present study, applying smaller shoulder abduction angles during (initiation of) functional reach, a potential coupling would be less prominent.

On the other hand, Ellis et al. observed that in an isometric situation an abnormal coupling between shoulder abduction and elbow flexion was present with the upper arm positioned in either $70^{\circ}$ or $20^{\circ}$ of shoulder abduction. ${ }^{14}$ McCrea et al. investigated reaching strategies of chronic stroke patients, applying a more functional movement of sagittal forward and upward reach, which is comparable to the reaching movement in the present study in terms of required (initial) shoulder elevation. ${ }^{15}$ They also did observe an abnormal coupling between shoulder and elbow movements: limitations in shoulder flexion were accompanied by increased shoulder abduction and increased elbow flexion. ${ }^{15}$

Besides stroke severity and arm position, differences in movement speed may also play a role in the occurrence of abnormal coupling between shoulder elevation and elbow flexion. In the reaching tasks used to study abnormal coupling in dynamic situations in before-mentioned studies of Beer, Dewald and colleagues, subjects were instructed to move as rapidly as possible. ${ }^{6,11-13}$ Also the study by McCrea et al. required maximal movement speed. ${ }^{15}$ In the present study, movement speed was lower by asking subjects to move at a comfortable, selfselected speed. Besides a potential influence of hyperreflexivity and spasticity 
during very fast movements, a high movement speed poses a larger strain on the neuromuscular system than the movement task in the present study, which may elicit a more pronounced abnormal synergistic coupling. Remarkably, reductions in elbow extension with increasing shoulder abduction torques have been observed during slow circular movements in a study investigating reductions in workspace applying a variety of sub- and supra-maximal shoulder abduction torques. ${ }^{16}$ However, this study involved an arm position of $90^{\circ}$ shoulder abduction, requiring larger shoulder abduction torques throughout the movement task than the present study involving a more functional arm movement.

Considering the findings of the present study in the context of above-mentioned research, it is plausible that abnormal coupling between shoulder and elbow movements in chronic stroke patients only limits movement performance when a strenuous task has to be performed, either with near-maximal force or with nearmaximal speed, or both. The present findings suggest that during sub-maximal, functional movements at lower velocities, abnormal synergistic coupling between shoulder and elbow movements is not predominant in either movement performance or muscle activation in mildly affected chronic stroke patients.

It has been shown that the ability to control movement direction throughout the workspace during functional reaching movements, similar to those in the present study, is preserved in chronic stroke patients, even though reaching distance, velocity, smoothness and straightness are compromised. ${ }^{17}$ Another study suggested that this may be associated with stroke severity. ${ }^{18}$ Less affected chronic stroke patients retain the ability to control arm movements according to the desired movement direction, whereas severely affected chronic stroke patients show a limitation of aimed arm movements to two gross directions (medial and lateral). ${ }^{18}$

This supports the observation that limitations in movement coordination, plausibly by abnormal coupling of shoulder and elbow movements, are only evident when the task demands are high enough to cross a certain level of effort, depending on the capacity of each stroke patient. In more severely affected stroke patients, functional activities corresponding with daily life may already approach their maximal level of motor control, eliciting an abnormal coupling between multiple muscle groups due to increased neural drive, whereas in less severely affected stroke patients such activities may not reach this critical effort level.

\section{Conclusions}

The present study suggests that for mildly affected chronic stroke patients, 
limitations in isolated, selective movement control may not play a major role in reducing functional arm use. For this group of patients, it would be valuable to asses which (other) impairments may be involved in limiting arm function, such as muscle weakness or incoordination, ${ }^{19}$ and to target these impairments specifically to increase functional gains. 


\section{References}

1. Kwakkel G, Kollen BJ, Van der Grond J, Prevo AJH. Probability of regaining dexterity in the flaccid upper limb: impact of severity of paresis and time since onset in acute stroke. Stroke 2003;34:2181-2186

2. Krakauer JW. Arm function after stroke: from physiology to recovery. Semin Neurol 2005;25(4):384-395

3. Brunnstrom S. Movement therapy in hemiplegia, a neurophysiological approach. New York: Harper \& Row, Publishers; 1970

4. Dewald JPA, Pope PS, Given JD, Buchanan TS, Rymer WZ. Abnormal muscle coactivation patterns during isometric torque generation at the elbow and shoulder in hemiparetic subjects. Brain 1995;118:495-510

5. Beer RF, Given JD, Dewald JPA. Task-dependent weakness at the elbow in patients with hemiparesis. Arch Phys Med Rehabil 1999;80:766-772

6. Beer RF, Dewald JPA, Dawson ML, Rymer WZ. Target-dependent differences between free and constrained arm movements in chronic hemiparesis. Exp Brain Res 2004; 156:458-470

7. Stienen AHA, Hekman EEG, Van der Helm FCT, Prange GB, Jannink MJA, Aalsma AMM, and Van der Kooij H. Dampace: dynamic force-coordination trainer for the upper extremities. In: Proceedings of the 10th International Conference on Rehabilitation Robotics (ICORR); June 13-15, 2007, Noordwijk aan Zee, the Netherlands:820-826

8. Hermens HJ, Freriks B, Disselhorst-Klug C, Rau G. Development of recommendations for sEMG sensors and sensor placement procedures. J Electromyogr Kinesiol 2000;10(5):361-374

9. Wu G, Van der Helm FC, Veeger $\mathrm{HE}$, et al. ISB recommendation on definitions of joint coordinate systems of various joints for the reporting of human joint motion Part II: shoulder, elbow, wrist and hand. J Biomech 2005;38(5):981-992

10. Ellis MD, Holubar BG, Acosta AM, Beer RF, Dewald JPA. Modifiability of abnormal isometric elbow and shoulder joint torque coupling after stroke. Muscle Nerve 2005;32:170-178

11. Beer RF, Dewald JPA, Rymer WZ. Deficits in the coordinaton of multijoint arm movements in patients with hemiparesis: evidence for disturbed control of limb dynamics. Exp Brain Res 2000;131:305-319

12. Dewald JPA, Sheshadri V, Dawson ML, Beer RF. Upper-limb discoordination in hemiparetic stroke: implications for neurorehabilitation. Top Stroke Rehabil 2001;8(1):1-12

13. Beer RF, Ellis MD, Holubar BG, Dewald JPA. Impact of gravity loading on post-stroke reaching and its relationship to weakness. Muscle Nerve 2007;36:242-250

14. Ellis MD, Acosta AM, Yao J, Dewald JPA. Position-dependent torque coupling and 
associated muscle activation in the hemiparetic upper extremity. Exp Brain Res 2007;176(4):594-602

15. McCrea PH, Eng JJ, Hodgson AJ. Saturated muscle activation contributes to compensatory reaching strategies after stroke. J Neurophysiol 2005;94:2999-3008

16. Sukal TM, Ellis MD, Dewald JPA. Shoulder abduction-induced reductions in reaching work area following hemiparetic stroke: neuroscientific implications. Exp Brain Res 2007;183:215-223

17. Kamper DG, McKenna-Cole AN, Kahn LE, Reinkensmeyer DJ. Alterations in reaching after stroke and their relation to movement direction and impairment severity. Arch Phys Med Rehabil 2002;83:702-707

18. Reinkensmeyer DJ, McKenna Cole A, Kahn LE, Kamper DG. Directional control of reaching is preserved following mild/moderate stroke and stochastically constrained following severe stroke. Exp Brain Res 2002;143:525-530

19. Wagner JM, Lang CE, Sahrmann SA, et al. Relationships between sensorimotor impairments and reaching deficits in acute hemiparesis. Neurorehabil Neural Repair 2006;20:406-416 


\section{Chapter $_{6}$}

CHANGES IN MUSCLE ACTIVATION AFTER REACH

TRAINING WITH GRAVITY COMPENSATION

IN CHRONIC STROKE PATIENTS

GB Prange

T Krabben

GJ Renzenbrink

MJ IJzerman

HJ Hermens

MJA Jannink

submitted 2009 


\section{Abstract}

Background - After stroke, involuntary coupling of shoulder abduction and elbow flexion can be reduced instantaneously by application of arm support, increasing range of motion. Unsupported reach may be improved by application of gravity compensation as training intervention, but underlying mechanisms are largely unknown.

Objective - To examine changes in muscle activation underlying changes in arm movement ability after gravity compensation training.

Methods - During 6 weeks, 8 chronic stroke patients received 18 sessions of 30 minutes gravity compensated reach training in combination with a rehabilitation game. Unsupported arm movement ability was assessed before and after training by blinded researchers using Fugl-Meyer (FM) assessment of motor status and reach performance. During unsupported reach, maximal distance, joint angles and muscle activity of 8 shoulder and elbow muscles were measured. Pre-post differences were calculated for all outcome measures.

Results - After gravity compensation training, FM and maximal reach distance improved in a majority of chronic stroke patients (on average +3.3 points and $+3.6 \%$ of arm length, respectively), together with increased elbow extension (mean $+9.2^{\circ}$ ) and shoulder elevation (mean $+4.6^{\circ}$ ). These improvements were accompanied predominantly by increased muscle activity of agonist muscles by at least $+20 \%$ with respect to pre-training levels.

Conclusions - The present findings indicate that improvement in unsupported reach distance after gravity compensation training in chronic stroke patients with mild to severe hemiparesis involved an increased activation of prime movers at the shoulder and elbow. This suggests that neurophysiological motor relearning processes can be stimulated in chronic stroke patients. 


\section{Introduction}

At least $90 \%$ of stroke survivors have to cope with limitations in arm function, often compromising activities of daily life. ${ }^{1}$ After a stroke, the ability to activate and contract muscles appropriately for a motor task is often impaired. Stereotypical patterns of involuntary coupling of movements are often observed, limiting selectivity of movements. ${ }^{2-4}$

To stimulate restoration of hemiparetic arm function, a training program should incorporate motivating, task-specific exercises involving active initiation and execution of functional tasks at a high intensity. ${ }^{5-7} \mathrm{~A}$ promising intervention to provide these key aspects is the application of robotic devices. A systematic review has shown that robot-aided therapy leads to improvements in motor function of the arm. ${ }^{8,9}$ However, it is not clear yet what aspects of such robot-aided approaches (e.g., passive, assisted or resisted movement) are major contributors to the effect on motor recovery of the arm and whether there is one single aspect that can make a valuable contribution. ${ }^{8}$

A basic aspect incorporated in the design of most robotic devices is arm support. ${ }^{10}$ Research indicates that the range of elbow extension of chronic stroke patients is larger during 2D planar reach with the arm supported at shoulder height by a device compared to unsupported reaching movements. ${ }^{11}$ This may be related to an involuntary, abnormal coupling (i.e., synergy) of predominantly shoulder abduction and elbow flexion, ${ }^{12}$ leading to reduced simultaneous elbow flexion torques when the amount of shoulder abduction needed to lift the arm is decreased by arm support. ${ }^{11}$ Regarding more natural, 3D reaching movements, we found an increase in maximal reach distance with gravity compensation, ${ }^{13,14}$ using the Freebal device. ${ }^{15}$

Besides an instantaneous improvement in supported arm movement ability, a long(er) term application of gravity compensation also improves free, unsupported movements of the affected arm of stroke patients. ${ }^{16-18}$ This may be related to a reduced abnormal coupling (i.e., enhanced movement selectivity) of the shoulder and elbow. ${ }^{16}$

Whether such improvements involve recovery of lost function or adaptation through compensational strategies is still unknown. Insight in underlying neurophysiological mechanisms involved in improvement of unsupported arm function after arm support training is important for optimal planning of the application of arm support during either conventional or technology-oriented stroke 
Chapter 6

rehabilitation. Therefore, the objective of the present study is to examine changes in muscle activation underlying changes in arm movement ability after gravity compensation training, combined with a virtual gaming environment to provide a motivating context. 


\section{Methods}

\section{Subjects}

Ten stroke patients were recruited from a local rehabilitation center if they had suffered a single unilateral stroke in the left hemisphere more than 6 months ago, experienced limited shoulder and elbow movement ability without limitation of active range of motion by pain or other orthopedic or neuromuscular deformities, and if they could follow instructions. All subjects provided written informed consent. The study was approved by the local medical ethics committee.

\section{Gravity compensation training}

Subjects received 18 sessions of 30 minutes gravity compensation training over a period of 6 weeks ( 3 sessions per week). A trained physical therapist supervised all sessions. Changes in arm movement ability were evaluated before (on two occasions within 2 weeks) and after training (within 1 week) by researchers blinded to training progress.

The training consisted of goal-directed reaching movements with gravity compensation in a virtual gaming environment (see figure 6.1). The weight of the subject's arm was counterbalanced by the Freebal device, designed to provide adjustable gravity compensation during 3D movements via ideal spring mechanisms. ${ }^{15}$ With increasing performance during the training, as determined by the physical therapist, the level of gravity compensation was decreased stepwise.

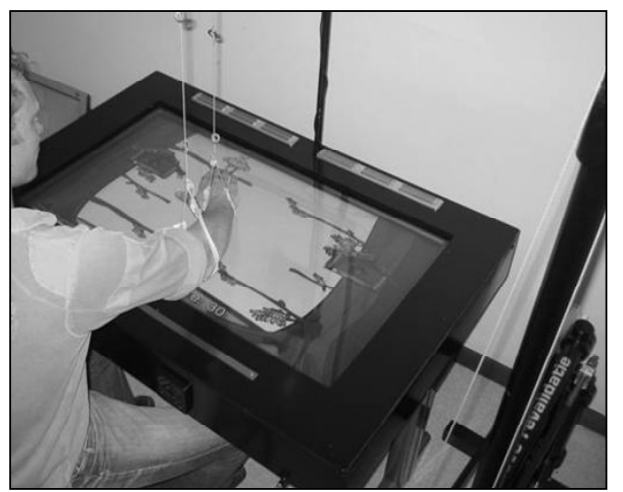

Figure 6.1 Set-up for gravity compensation training

Notes: Gravity compensation device 'Freebal' (on the right) and virtual gaming environment 'FurballHunt' (on the table display). 
In the virtual gaming environment designed for the present study, FurballHunt, patients had to chase away birds from a tree branch by reaching for them with their hand; the faster the birds were chased, the more points were awarded. ${ }^{19}$ The game was displayed on a horizontally placed television screen, creating a table display, from which arm movements were detected using a webcam (Logitech Quickcam Messenger; Logitech Inc., Fremont (CA), USA) placed above the table display and were identified by motion capturing software (custom written in Flash 8; Adobe Systems Inc., San Francisco (CA), USA).

\section{Evaluation}

Motor status of the hemiparetic arm was evaluated before and after training using the upper extremity part of the Fugl-Meyer (FM) assessment (maximal score of 66 points indicates fully selective movements). ${ }^{4}$

Movement performance was evaluated during an unsupported maximal reaching movement to a target placed beyond arm's length in front of the acromion of the hemiparetic arm at elbow height (in the sagittal plane). To minimize trunk and shoulder movements, the subject was strapped to the chair with a four point safety belt. The starting position of subjects during the reaching task was with the upper arm aligned with the trunk and the forearm pointing straight forward. Each subject was instructed to perform 5 reaching movements as far and straight forward as possible at a comfortable, self-selected speed.

During the maximal reach task, bi-polar surface electromyography (EMG) was recorded from 8 muscles, according to the guidelines of the SENIAM project. ${ }^{20}$ EMG signals of biceps (BIC), long and lateral head of triceps (TILO and TILA), anterior deltoid $(A D)$, posterior deltoid (PD), upper part of pectoralis major (PEC), lattissimus dorsi (LD) and upper trapezius (TRA) were recorded using a 16-channel Porti system (TMSI, Oldenzaal, the Netherlands), band-pass filtered ( $2^{\text {nd }}$ order zero phase shift Butterworth, cut-off frequencies $10-400 \mathrm{~Hz}$ ) and converted to smooth rectified EMG (SRE) signals (using a low-pass $2^{\text {nd }}$ order zero phase shift Butterworth filter at $25 \mathrm{~Hz}$ for smoothing).

Joint angles of the arm were defined according to recommendations of the International Society of Biomechanics, ${ }^{21}$ and recorded with the use of an instrumented, passive exoskeleton device, Dampace. ${ }^{22}$ Angular sensors measured transversal rotation (shoulder plane of elevation (SP): angle of humerus with virtual line through shoulders; humerus pointing straight forward represents $0^{\circ}$ and pointing laterally $-90^{\circ}$ ), upper arm elevation (shoulder angle of elevation (SE): angle between humerus and trunk in the plane of elevation; humerus aligned with 
trunk represents $0^{\circ}$ ) and elbow (E) flexion/extension (upper arm extended denotes $\left.180^{\circ}\right)$. Joint angles were sampled at $1000 \mathrm{~Hz}$, low-pass filtered ( $1^{\text {st }}$ order Butterworth filter, cut-off frequency $40 \mathrm{~Hz}$ ) and stored on a computer at $50 \mathrm{~Hz}$.

In addition, lengths of upper arm (between acromion and lateral epicondyle of humerus) and forearm (between lateral epicondyle of humerus and third metacarpophalangeal joint) were measured to determine joint positions.

\section{Data analysis}

The position of the hand was calculated relative to the position of the shoulder to exclude shoulder and trunk movements from the reaching movements. The maximal distance between acromion and third metacarpophalangeal joint represented maximal reach distance; the distance travelled by the hand from start to finish of reach represents the range of reach, expressed as percentage of arm length. The direction of reach is represented by the angle of deviation from the desired reach direction straight forward (positive values indicate medial deviation). Movement velocity of the hand (in $\mathrm{cm} / \mathrm{s}$ ) was also calculated. Minimal (min) and maximal (max) joint angles at the start and end of reach were used to determine ranges of motion (ROM) of the shoulder and elbow (in ${ }^{\circ}$ ) during reach.

Of the 5 repetitions, EMG and kinematic data of the 3 furthest reaches were averaged and displayed synchronously. The duration of the reaching movement was determined by identifying minimal (start of reach) and maximal (end of reach) elbow joint angles and expressed as $100 \%$, to account for intra- and inter-subject variation. A typical example is displayed in figure 6.2. After initial qualitative inspection of muscle activation patterns, the level of muscle activity was quantified by calculating the mean SRE-value during the averaged reaching movement for each muscle. Co-contraction ratios were calculated to examine individual intermuscle coupling between BIC and TILA, BIC and TILO, PD and AD, BIC and AD:

$C C=\frac{\overline{S R E}_{\text {antagonist }}}{\overline{S R E}_{\text {agonist }}}$

\section{Statistical analysis}

Initial analysis of the data obtained during the two pre-training evaluations revealed no clear trend in baseline outcome. Therefore, pre-training data of the 2 sessions were averaged per subject. For all outcome measures, changes before and after training were calculated within each subject. These change scores were averaged over all subjects and the corresponding 95\% confidence intervals (95\%- 
CI) were calculated to identify significant pre-post differences. Within-subject relations between outcome measures were examined using scatter plots and Pearson's correlation coefficients. Since the nature of the present study is explorative and includes a relatively small sample of subjects, no additional statistical tests for repeated measures were performed.
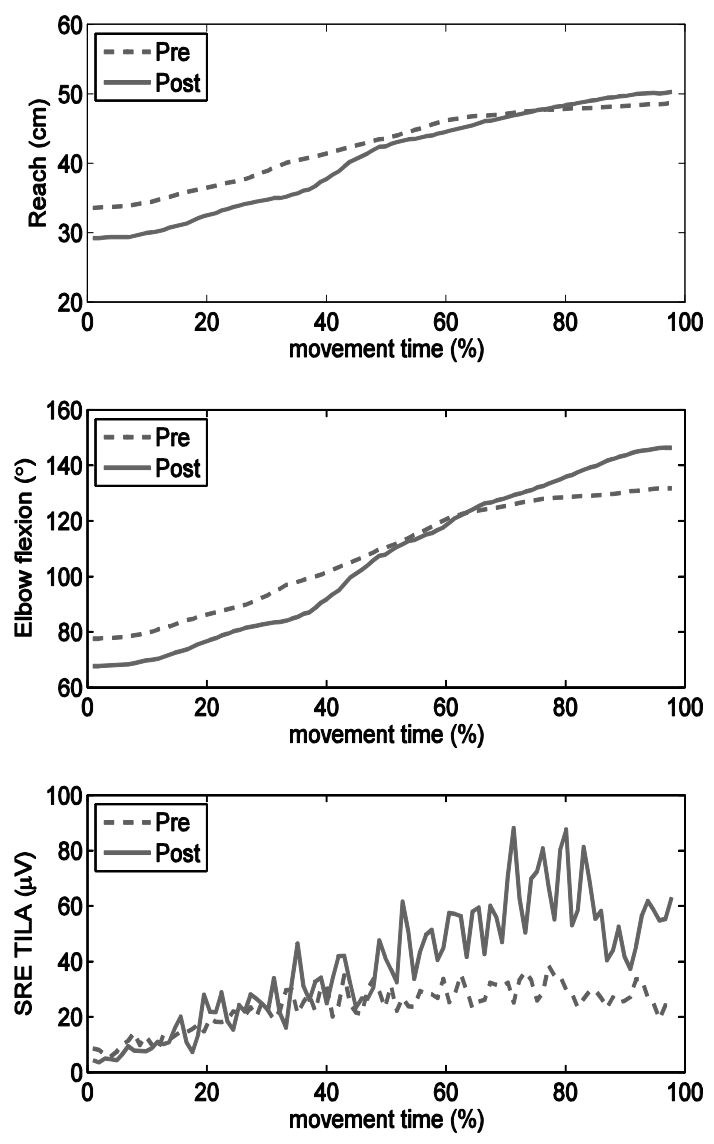

Figure 6.2 Overview of reach and muscle activation data

Notes: Typical example (S2) of reach distance, elbow extension and muscle activity of lateral head of triceps (TILA) before (light lines) and after (dark lines) gravity compensation training. 


\section{Results}

\section{Subject characteristics}

Ten chronic stroke patients were included in the present study, of which 1 patient did not complete the training period due to time constraints (S3). Another patient could not fulfill the evaluation task requirements (S10). Of the remaining 8 stroke patients (table 6.1), 3 were male and 5 were female. All had suffered first-ever ischemic strokes; in 7 patients the medial cerebral artery region was affected, the other patient (S6) suffered a cerebellar infarction. Mean time post-stroke was 30 months (range 8-58 months). Two of the 8 chronic stroke patients (S1 and S2) received conventional occupational therapy besides gravity compensation training in an out-patient setting. Initial stroke severity ranged from 7 to 61 points on the FM score. Mild hemiparesis ( $F M \geq 45)$ was present in 3 patients, 2 patients had moderate hemiparesis $(20<\mathrm{FM}<45)$ and 3 patients were severely affected $(\mathrm{FM} \leq 20)$.

\section{Changes in motor status}

After 6 weeks of gravity compensation training, in 7 of 8 stroke patients FM scores had increased (table 6.1). In 3 of those 7 patients, the increase in FM score ranged from 6.5 to 8 points. The average increase of all 8 stroke patients was 3.3 points (95\%-CI $=-0.2$ to 6.7$)$. When excluding the outlier with decreased FM score, the average increase was 4.4 points, achieving significance with a $95 \%-C I$ of 1.7 to 7.0. Improvements in motor status were not limited to a certain category of stroke severity, but included patients with mild, moderate and severe hemiparesis (table $6.1)$.

Table 6.1 Subject characteristics and changes in Fugl-Meyer (FM) scores after training

\begin{tabular}{l|lllll}
\hline Subject & Sex & Time post-stroke & pre FM* & post FM & $\mathbf{\Delta ~ F M}$ \\
\hline 1 & M & 58 months & 12.0 & 20 & +8.0 \\
2 & F & 13 months & 45.5 & 53 & +7.5 \\
4 & F & 27 months & 10.0 & 11 & +1.0 \\
5 & F & 24 months & 44.5 & 51 & +6.5 \\
6 & M & 30 months & 61.0 & 64 & +3.0 \\
7 & F & 39 months & 45.5 & 47 & +1.5 \\
8 & F & 39 months & 7.0 & 10 & +3.0 \\
9 & M & 8 months & 25.5 & 21 & -4.5 \\
\hline
\end{tabular}

$*$ average value of the first two baseline measurements 


\section{Changes in reach performance}

Movement speed was largely unchanged after gravity compensation training $(p \geq 0.780)$, with an average movement speed of $5 \mathrm{~cm} / \mathrm{sec}$. After training, movement performance of unsupported reach had improved (up to $+13.5 \%$ of arm length) in all but 1 stroke patient, via either an improved maximal distance or range of reach, or both (table 6.2). On average (table 6.2), maximal reach distance increased by $3.6 \%( \pm 1.5)$ of arm length (corresponding with $+2.4 \mathrm{~cm})$ after training $(95 \% \mathrm{CI}=-0.08$ to 7.17$)$. Average range of reach increased by $4.9 \%( \pm 2.2)$ of arm length $(+3.1 \mathrm{~cm})$, with a corresponding $95 \%$-CI of -0.36 to 10.19 . The average direction of reach showed a very small decrease of $0.8^{\circ}$, indicating that the deviation of movement direction remained near pre-training levels.

Table 6.2 Mean reach distance and direction of each subject before and after training

\begin{tabular}{|c|c|c|c|c|c|c|c|c|c|}
\hline \multirow[b]{2}{*}{ Subject } & \multicolumn{3}{|c|}{ Max reach $(\%) *$} & \multicolumn{3}{|c|}{ ROM reach (\%)* } & \multicolumn{3}{|c|}{ Dir reach $\left({ }^{\circ}\right) \#$} \\
\hline & pre & post & $\Delta$ & pre & post & $\Delta$ & pre & post & $\Delta$ \\
\hline 1 & 54.5 & 55.6 & +1.1 & 30.6 & 35.9 & +5.3 & 10.9 & 10.7 & -0.2 \\
\hline 2 & 80.2 & 83.8 & +3.6 & 79.2 & 91.2 & +12.0 & 7.6 & 5.3 & -2.3 \\
\hline 4 & 44.0 & 40.1 & -3.9 & 32.8 & 31.0 & -1.8 & 16.3 & 27.0 & +10.7 \\
\hline 5 & 83.8 & 85.3 & +1.5 & 68.1 & 65.8 & -2.3 & 3.4 & 3.8 & +0.4 \\
\hline 6 & 91.6 & 93.6 & +2.0 & 97.9 & 101.9 & +4.0 & 3.6 & 5.5 & +1.9 \\
\hline 7 & 69.3 & 77.6 & +8.3 & 53.4 & 66.9 & +13.5 & 4.7 & 3.3 & -1.4 \\
\hline 8 & 47.0 & 55.6 & +8.6 & 32.9 & 42.6 & +9.7 & 40.2 & 26.5 & -13.7 \\
\hline 9 & 72.1 & 79.3 & +7.2 & 51.5 & 50.4 & -1.1 & 17.3 & 15.8 & -1.5 \\
\hline $\begin{array}{l}\text { mean } \\
\left(\% \text { or }{ }^{\circ}\right) \\
( \pm s d)\end{array}$ & $\begin{array}{l}67.8 \\
(17.6)\end{array}$ & $\begin{array}{l}71.4 \\
(18.6)\end{array}$ & $\begin{array}{l}+3.6 \\
(4.3)\end{array}$ & $\begin{array}{l}55.8 \\
(24.4)\end{array}$ & $\begin{array}{l}60.7 \\
(25.7)\end{array}$ & $\begin{array}{l}+4.9 \\
(6.3)\end{array}$ & $\begin{array}{l}13.0 \\
(12.3)\end{array}$ & $\begin{array}{l}12.2 \\
(9.9)\end{array}$ & $\begin{array}{l}-0.8 \\
(6.7)\end{array}$ \\
\hline $\begin{array}{l}\text { mean } \\
(\mathrm{cm}) \\
( \pm \mathrm{sd})\end{array}$ & $\begin{array}{l}45.6 \\
(12.8)\end{array}$ & $\begin{array}{l}48.0 \\
(13.6)\end{array}$ & $\begin{array}{l}+2.4 \\
(2.9)\end{array}$ & $\begin{array}{l}37.3 \\
(16.1)\end{array}$ & $\begin{array}{l}40.4 \\
(16.4)\end{array}$ & $\begin{array}{l}+3.1 \\
(4.0)\end{array}$ & - & - & - \\
\hline
\end{tabular}

* Reach distance (max) and range of motion (ROM) is represented as percentage of arm length, except for mean values displayed in $\mathrm{cm}$ in the bottom row

\# Reach direction (dir) is represented as angular deviation from the desired direction

\section{Changes in joint rotations}

After training, all but 1 stroke patient had improved their elbow excursion (up to $+24.7^{\circ}$ ) during unsupported reach, by increasing maximal elbow extension or elbow ROM, or both. About half of the stroke patients had increased their maximal shoulder elevation angle (up to $+17 \cdot 6^{\circ}$ ). Maximal shoulder plane of elevation increased in 6 stroke patients (up to $+18.6^{\circ}$ ). On group level (figure 6.3), elbow 
ROM had increased significantly by $+9.2^{\circ}( \pm 3.6)$, with a corresponding $95 \%$-CI of 0.7 to 17.7. The upper arm was held in a more elevated $(+4.6 \% ; 95 \%-\mathrm{CI}=-0.4$ to $9.5)$ and more forward directed $(+8.70 ; 95 \%-\mathrm{CI}=2.2$ to 15.3$)$ position throughout the reaching movement, even though ROM of shoulder elevation and shoulder plane did not change substantially within patients.

\section{Changes in muscle activation}

Changes in muscle activation during unsupported reach after gravity compensation training were mainly observed in the prime movers at the shoulder and elbow. Mean SRE-values of TILA and/or TILO had increased in 6 of the 8 stroke patients ( +10.1 up to $+68.9 \mu \mathrm{V}$ and +4.4 up to $+30.7 \mu \mathrm{V}$, respectively), and of $A D$ in 5 of the 8 stroke patients $(+4.6$ up to $+53.6 \mu \mathrm{V})$. In the other muscles, only small or inconsistent changes across subjects were observed. On group level (figure 6.4), TILA activity had increased by $21.6 \mu \mathrm{V}(=+68 \%)$, with a corresponding $95 \%$-CI of -0.37 to 43.6. The increase in TILO activity was somewhat less pronounced on group level with $9.1 \mu \mathrm{V}(=+35 \%)$, with a corresponding $95 \%-\mathrm{CI}$ of -1.6 to 19.9 . AD increased on average $9.6 \mu \mathrm{V}(=+20 \%)$, with a corresponding $95 \%-\mathrm{CI}$ of -6.9 to $26.1 \mu \mathrm{V}$.

\section{Changes in co-contraction}

In 4 out of 8 stroke patients, $\mathrm{CC}_{\mathrm{BIC} / \mathrm{TILA}}$ was reduced after gravity compensation training. In 3 of them, $\mathrm{CC}_{\mathrm{BIC} / \mathrm{TLO}}$ had also decreased. In addition, $\mathrm{CC}_{\mathrm{PD} / \mathrm{AD}}$ had decreased in 7 stroke patients. The change in $\mathrm{CC}_{\mathrm{BIC} / \mathrm{AD}}$ varied largely between

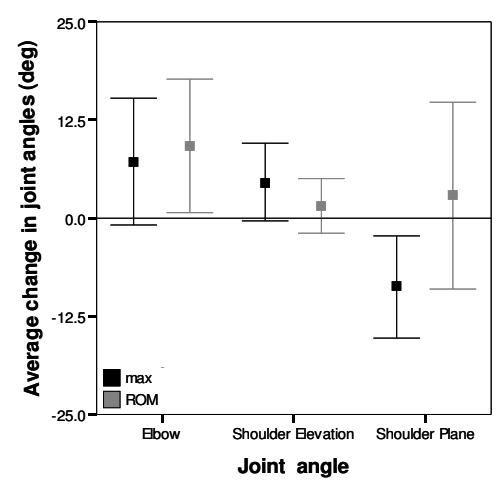

Figure 6.3 Change in joint excursions after gravity compensation training

Notes: Average changes $( \pm 95 \%-\mathrm{CI})$ in maximum (dark symbols) and range (light symbols) of joint excursions within patients after 6 weeks of gravity compensation training. 
patients, with a reduction in 3 patients, an increase in 2 patients and no substantial change in 3 stroke patients. On group level, $\mathrm{CC}_{\mathrm{BIC} / \mathrm{TLA}}$ and $\mathrm{CC}_{\mathrm{PD} / \mathrm{AD}}$ had decreased by 0.2 . Despite the inconsistency in change in $\mathrm{CC}_{\mathrm{BIC} / \mathrm{AD}}$ across patients, the average ratio over all 8 patients decreased by 0.3 . None of these differences were statistically significant according to the $95 \%$ confidence interval. These findings show a trend towards less resistance of the prime movers at the shoulder and elbow by their antagonists after gravity compensation training. In 3 of the 8 chronic stroke patients activity of AD was accompanied by a smaller amount of BIC activity simultaneously, suggesting a less strong abnormal coupling of S-elevation and E-flexion after gravity compensation training in those patients. However, in the majority of patients this influence was not found.

\section{Correlations between movement performance and muscle activity}

Before and after training, reach distance was positively correlated with joint excursion of $E$, SE and SP separately $(\rho \geq 0.72)$. Also, increases in E-max were correlated rather strongly $(\rho \geq 0.67)$ with increases in reach distance and increases in E-ROM were accompanied by increases in range of reach. This indicates that improvements in reach distance were individually related to improvements in elbow extension.

When looking at within-subject correlations concerning muscle activation, positive correlations between E-ROM and SRE-values of TILO were observed

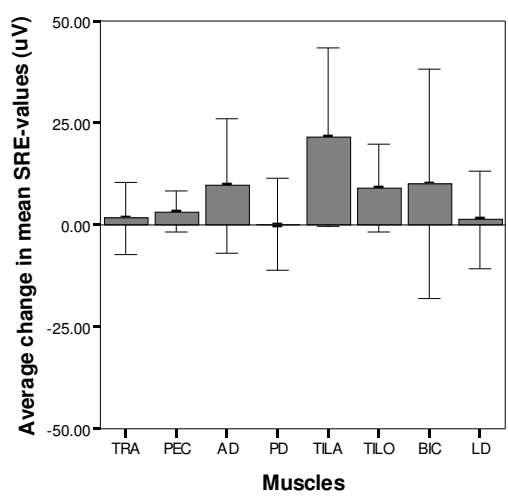

Figure 6.4 Change in smooth rectified EMG (SRE) values after training

Notes: Average changes $( \pm 95 \%-\mathrm{CI})$ in mean smooth rectified EMG (SRE) values per muscle within patients after gravity compensation training; TRA = upper trapezius; $\mathrm{PEC}=$ pectoralis major; $\mathrm{AD}=$ anterior deltoid; $\mathrm{PD}=$ posterior deltoid; TILA = lateral head of triceps; TILO = long head of triceps; $\mathrm{BIC}=$ biceps; $\mathrm{LD}=$ lattissimus dorsi 
before and after training ( $\rho \geq 0.67)$, in addition to positive correlations of SREvalues of both TILO and TILA with SE-max and SE-ROM $(\rho \geq 0.70)$. However, increases in reach distance due to gravity compensation training were not strongly correlated with increases in SRE-values of TILA and/or TILO (or any other muscle). The same was observed for increases in elbow excursion after training, or increases in other EMG parameters. This is probably due to the small sample of participants and a considerable amount of variation between patients. 


\section{Discussion}

\section{Improved reach performance after gravity compensation training}

After 6 weeks of training with gravity compensation, improvements in unsupported reach distance were accompanied by an increased activation of prime movers at the shoulder and elbow. Motor status, as assessed by FM, improved by 3.3 points on average after gravity compensation training. This increase is somewhat mitigated due to the one patient with a rather prominent decrease in FM score, plausibly due to day-to-day variability in arm function. The improvement in FM score was as large as 6.5 up to 8 points in 3 of the 8 chronic stroke patients, indicating a clinically relevant improvement (i.e., an increase of $10 \%$ of maximal FM score) ${ }^{23}$ Reach distance increased in a majority of stroke patients (on average $3.1 \mathrm{~cm}$ active ROM), together with a larger range of elbow extension and a more elevated and forward directed position of the upper arm. Two of the 3 patients with the largest FM increase received additional conventional occupational therapy. However, these patients did not display the largest increases in reach performance, suggesting that the influence of added therapy on the results is limited in this case.

The improvements in arm movement ability due to gravity compensation training observed in the present study are along the same lines as found in studies applying other types of arm support. A pilot study applying arm support via a passive exoskeleton instrumented with elastic bands (T-WREX) in 5 chronic stroke patients resulted on average in an improved motor status of 5 points on FM assessment and an increased reaching distance of $3.2 \mathrm{~cm} .{ }^{16}$ Another study using T-WREX with a larger group of 15 stroke patients showed an average increase in FM score of 3.3 points and an improved reach distance by $2.8 \mathrm{~cm} .{ }^{17}$ In addition, reach training via de-weighting of the arm applying sling suspension resulted in an increased motor status of the affected arm of approximately 2.7 points on FM score in a group of 31 chronic stroke patients. ${ }^{18}$

Improvements in motor status after gravity compensation training are also comparable to improvements after robot-aided therapy, with a pooled mean increase in FM score of 3.7 points. $^{8}$ This suggests that the application of gravity compensation alone, using a relatively simple device, may be as beneficial to improve arm movement ability as the application of rehabilitation robotics through more complex devices. 


\section{Incorporation of virtual reality}

Studies investigating the effect of arm support differed concerning training intensity and task-specificity. A high training intensity and task-specificity of a training program is beneficial for stimulation of motor recovery after stroke..$^{5-7}$ The studies using T-WREX involved the highest training intensity and exercises that resembled functional activities the most (i.e., virtual activities of daily living including hand function) ${ }^{16,17}$ in comparison with the present study and the study by Amirabdollahian et al. ${ }^{18}$ Accordingly, the T-WREX studies showed somewhat more pronounced improvements in motor status. ${ }^{16,17}$

To increase task-specificity, virtual reality is used in the T-WREX studies. ${ }^{16}$ Dexterity of the hand is simulated during virtual functional exercises via a simpler action (i.e., hand grip) performed by the patients in real life. In the present study, gravity compensation training involved a virtual gaming environment to enhance motivation during training. In both applications of VR, additional information about the result or the performance of the movement is provided. Such augmented feedback can stimulate motor (re)learning processes after stroke. ${ }^{24}$ Remarkably, the results of a comparison of augmented T-WREX training to table top exercises are inconclusive. ${ }^{17}$ In the present study, the potential added value of augmented feedback can not be distinguished from the influence of gravity compensation. Although the present study and the T-WREX studies ${ }^{16}$ do indicate that it is feasible to combine arm support training with virtual reality to design appropriate training environments, more research is needed to identify its optimal application in order to enhance the potential of such interventions.

\section{Underlying neurophysiological mechanisms}

From cross-sectional studies is known that arm support reduces an abnormal coupling between shoulder abduction and elbow flexion during isometric contractions. ${ }^{12}$ In the present longitudinal study, we have observed improvements in unsupported reaching movements after a longer-term application of gravity compensation, accompanied by an increased shoulder elevation throughout the reaching movement and an increased range of elbow extension. Sanchez et al. and Housman et al. also observed increases in unsupported reaching distance, suggesting an improved selectivity of shoulder and elbow movements. ${ }^{16,17}$ Nevertheless, other mechanisms than a reduction in abnormal coupling may also result in such improvements in reach performance, as seen from the changes in muscle activation.

Improvements in reach performance after gravity compensation training were 
accompanied by more pronounced muscle activity of the prime movers. In addition, co-contraction of antagonist muscles at the shoulder and elbow was reduced to some extent. However, it is likely that the reduction in antagonist/agonist ratio is at least partly related to the increased level of agonist activation. Reduction of co-activation of muscles thought to act together in abnormal synergies after stroke (mainly BIC and AD in this case), ${ }^{25}$ was only observed in a few stroke patients (ranging from severely to mildly affected) after gravity compensation training. It is possible that the functional reaching movement in the present study did not require sufficient shoulder elevation torque to provoke a strong coupling that limited reaching performance, as found in a previous study (submitted for publication). ${ }^{26}$ Either way, improvements in functional reaching movements seen after gravity compensation training in the present group of chronic stroke patients seem to be mainly related to improved coordination of elbow extensors, suggesting a main role for stimulation of control of prime movers of the upper extremity. This is supported by a study showing that improved reach performance during the first months of recovery from stroke was predominantly related to improved agonist coordination and contraction, but not to reduced cocontraction. ${ }^{27}$

\section{Clinical implications}

The findings of the present explorative study are promising for the application of gravity compensation in stroke rehabilitation. In this case, training intensity was not even very high, indicating that the potential may be even greater, when considering that an increase in training intensity will benefit restoration of arm function even more. ${ }^{28}$ Nonetheless, the results have to be interpreted with care due to a limited number of 8 participants. In addition, potential effects of an increase in overall training intensity can not be discerned. It may have been that stroke patients in the chronic phase have learned to avoid use of their affected arm. During gravity compensation training, they were forced to use the hemiparetic arm more, which should be taken into account in future research by including a control group. Despite this, the findings of this explorative study warrant further research into gravity compensation training and present several valuable implications.

Improvements in reach performance, motor status and neuromuscular activation after gravity compensation training were found in chronic stroke patients with not only mild and moderate, but also severe hemiparesis, indicating that this intervention may be valuable for stroke patients with a large range of stroke 
severity. Additionally, the facilitating influence of gravity compensation enables a large part of the stroke population to start active reaching exercises at an early phase during stroke rehabilitation. Furthermore, gravity compensation in combination with augmented feedback is a suitable way to provide active and taskspecific treatment without the need for high-tech devices. This implies that gravity compensation can be a feasible and valuable application in stroke rehabilitation.

\section{Conclusion}

The present explorative study suggests that gravity compensation training has the potential to improve unsupported reach performance of chronic stroke patients with impairments ranging from mild to severe hemiparesis. This improvement is predominantly related to an improved ability to activate agonist muscles, especially at the elbow, suggesting that neurophysiological motor relearning processes regarding the upper extremity can be stimulated in chronic stroke patients. 


\section{References}

1. Kwakkel G, Kollen BJ, Van der Grond J, Prevo AJH. Probability of regaining dexterity in the flaccid upper limb: impact of severity of paresis and time since onset in acute stroke. Stroke 2003;34:2181-2186

2. Brunnstrom S. Movement therapy in hemiplegia, a neurophysiological approach. New York: Harper \& Row, Publishers; 1970

3. Twitchell TE. The restoration of motor function following hemiplegia in man. Brain $1951 ; 74(4): 443-480$

4. Fugl-Meyer AR, Jääskö L, Leyman I, Olsson S, Steglind S. The post-stroke hemiplegic patient. A method for evaluation of physical performance. Scand J Rehab Med 1975;7:13-31

5. Kwakkel G, Wagenaar RC, Twisk JWR, Lankhorst GJ, Koetsier JC. Intensity of leg and arm training after primary middle-cerebral-artery stroke: a randomised trial. Lancet 1999;354:189-194

6. Feys HM, De Weerdt WJ, Selz BE, et al. Effect of a therapeutic intervention for the hemiplegic upper limb in the acute phase after stroke. A single-blind, randomized, controlled multicenter trial. Stroke 1998;29:785-792

7. Barreca S, Wolf SL, Fasoli S, Bohannon R. Treatment interventions for the paretic upper limb of stroke survivors: a critical review. Neurorehabil Neural Repair 2003; 17:220-226

8. Prange GB, Jannink MJA, Groothuis CGM, Hermens HJ, IJzerman MJ. A systematic review of the effect of robot-aided therapy on recovery of the hemiparetic arm after stroke. J Rehabil Res Dev 2006;43(2):171-184

9. Kwakkel G, Kollen BJ, Krebs HI. Effects of robot-assisted therapy on upper limb recovery after stroke: A systematic review. Neurorehabil Neural Repair 2008; 22(2):111-121

10. Johnson MJ. Recent trends in robot-assisted therapy environments to improve reallife functional performance after stroke. J NeuroEng Rehabil 2006;3(29)

11. Beer RF, Dewald JPA, Dawson ML, Rymer WZ. Target-dependent differences between free and constrained arm movements in chronic hemiparesis. Exp Brain Res 2004; 156:458-470

12. Beer RF, Dewald JPA, Rymer WZ. Deficits in the coordinaton of multijoint arm movements in patients with hemiparesis: evidence for disturbed control of limb dynamics. Exp Brain Res 2000;131:305-319

13. Prange GB, Stienen AHA, Jannink MJA, Van der Kooij H, IJzerman MJ, and Hermens $\mathrm{HJ}$. Increased range of motion and decreased muscle activity during maximal reach with gravity compensation in stroke patients. In: Proceedings of the 10th International Conference on Rehabilitation Robotics (ICORR); June 13-15, 2007, Noordwijk aan Zee, the Netherlands:467-471 
14. Stienen AHA, Van der Helm FCT, Prange GB, Jannink MJA, and Van der Kooij H. Effects of gravity compensation on the range-of-motion of the upper extremities in robotic rehabilitation after stroke. In: Proc Inter Shoulder Group; 2006, Oct 9-10, Chicago (IL), USA

15. Stienen AHA, Hekman EEG, Van der Helm FCT, Prange GB, Jannink MJA, Aalsma $A M M$, and Van der Kooij $H$. Freebal: dedicated gravity compensation for het upper extremities. In: Proceedings of the 10th International Conference on Rehabilitation Robotics (ICORR); June 13-15, 2007, Noordwijk aan Zee, the Netherlands:804-808

16. Sanchez RJ, Liu J, Rao S, et al. Automating arm movement training following severe stroke: functional exercises with quantitative feedback in a gravity-reduced environment. IEEE Trans Neural Syst Rehabil Eng 2006;14(3):378-389

17. Housman SJ, Scott KM, Reinkensmeyer DJ. A randomized controlled trial of gravitysupported, computer-enhanced arm exercise for individuals with severe hemiparesis. Neurorehabil Neural Repair 2009;23(5):505-514 (DOI:10.1177/1545968308331148)

18. Amirabdollahian F, Loureiro R, Gradwell E, Collin C, Harwin W, Johnson G. Multivariate analysis of the Fugl-Meyer outcome measures assessing the effectiveness of GENTLE/S robot-mediated stroke therapy. J NeuroEng Rehabil 2007;4(4)

19. Prange GB, Krabben T, Molier BI, Van der Kooij H, and Jannink MJA. A low-tech virtual reality application for training of upper extremity motor function in neurorehabilitation. In: Proceedings of Virtual Rehabilitation; 25-27 August 2008, Vancouver, Canada

20. Hermens HJ, Freriks B, Disselhorst-Klug C, Rau G. Development of recommendations for sEMG sensors and sensor placement procedures. J Electromyogr Kinesiol 2000; 10(5):361-374

21. Wu G, Van der Helm FC, Veeger HE, et al. ISB recommendation on definitions of joint coordinate systems of various joints for the reporting of human joint motion Part II: shoulder, elbow, wrist and hand. J Biomech 2005;38(5):981-992

22. Stienen AHA, Hekman EEG, Van der Helm FCT, Prange GB, Jannink MJA, Aalsma $A M M$, and Van der Kooij H. Dampace: dynamic force-coordination trainer for the upper extremities. In: Proceedings of the 10th International Conference on Rehabilitation Robotics (ICORR); June 13-15, 2007, Noordwijk aan Zee, the Netherlands:820-826

23. Gladstone DJ, Danells CJ, Black SE. The Fugl-Meyer assessment after stroke: a critical review of its measurement properties. Neurorehab Neural Repair 2002; 16(3):232-240

24. Winstein $\mathrm{CJ}$, Campbell Stewart J. Conditions of task practice for individuals with neurologic impairments. In: Selzer M, Clarke S, Cohen L, Duncan P, Gage F, eds. Textbook of neural repair and rehabilitation. Medical Neurorehabilitation Volume II; Cambridge, UK: Cambridge University Press; 2006: 89-102

25. Dewald JPA, Pope PS, Given JD, Buchanan TS, Rymer WZ. Abnormal muscle coactivation patterns during isometric torque generation at the elbow and shoulder in 
hemiparetic subjects. Brain 1995;118:495-510

26. Prange GB, Jannink MJA, Stienen AHA, Van der Kooij H, IJzerman MJ, Hermens $H J$. An explorative, cross-sectional study into abnormal muscle synergies during functional reach in chronic stroke patients. (submitted) 2009

27. Wagner JM, Dromerick AW, Sahrmann SA, Lang CE. Upper extremity muscle activation during recovery of reaching in subjects with post-stroke hemiparesis. Clin Neurophysiol 2007;118:164-176

28. Kwakkel G, Wagenaar RC, Koelman TW, Lankhorst GJ, Koetsier JC. Effects of intensity of rehabilitation after stroke. A research synthesis. Stroke 1997;28(8):15501556 
General Discussion 



\section{General Discussion}

Stroke is a major cause of permanent disability. ${ }^{1}$ A reduced arm and hand function may cause serious limitations in activities of daily living for the majority of stroke patients. ${ }^{2}$ Rehabilitation is provided to stimulate restoration of arm function after stroke, consisting for a large part of exercise therapy. For optimal results, active initiation and execution of movements, task-specificity and a high training intensity have been identified as key aspects. ${ }^{3}$ Besides restoration of degraded neural function via stimulation of cortical reorganization (i.e., restitution of function), improved functional ability of the hemiparetic arm can also involve compensatory strategies to circumvent lost motor function (i.e., substitution of function). ${ }^{4}$ Such strategies may mask the effects of 'true recovery', complicating the understanding of the mechanisms involved in restoration of arm function after stroke. Insight in those mechanisms and their relationship is essential to optimize the application of interventions in stroke rehabilitation. ${ }^{4}$

The development of innovative rehabilitation robotics has led to design of interventions that specifically take the key aspects for restoration of arm function into account. Robotic devices can particularly provide intensive, highly repetitive therapy, by assisting or resisting arm movements in various degrees depending on the abilities of the patient, during task-specific exercises. Although initial feasibility studies provided promising results, ${ }_{1}^{5}$ it is still unclear if the present manner of application of robot-aided therapy is the optimal approach to achieve maximal improvements in arm function. For example, the various therapy modalities applied by rehabilitation robotics (i.e., passive, active-assisted and active-resisted movements) engage the neuromuscular system to different extents, which may involve different mechanisms, some perhaps more effective than others, to improve arm function. Since many approaches intervene at the motor control level, muscle activation may change consequently. By investigating changes in muscle activation due to application of robotic devices, the merits of different approaches may be discerned. Such information can also aid in understanding which mechanisms of recovery are targeted by the application of rehabilitation robotics. This knowledge can then be used to determine how to take advantage of the merits of rehabilitation robotics Therefore, the main objective of the research reported in this $\mathrm{PhD}$ thesis is to obtain a better understanding of the impact of different therapy modalities of rehabilitation robotics on neuromuscular control of arm movements of stroke patients. 


\section{Current application of rehabilitation robotics after stroke}

In chapter 2, a systematic review is described that analyzes the effect of existing clinical studies into robot-aided rehabilitation. Although the findings indicate that the application of rehabilitation robotics is able to improve motor control of proximal arm movements of both sub-acute and chronic stroke patients, perhaps even more so than conventional therapy, the most valuable application could not be discerned. The treatment currently provided by rehabilitation robotics generally consists of a mix of passive, active-assisted and active-resisted movements, without looking into differential effects of those therapy modalities.

An exception is a study comparing active-assisted robot-aided therapy with active-resisted exercises, but no significant differences in motor recovery between active-assisted and active-resisted therapy were found. ${ }^{6}$ In another study, standardized robot-aided therapy was compared with a progressive training program, where robotic assistance was diminished as patients improved their ability to perform the training task. ${ }^{7}$ The findings favored the progressive training group, which confirms that adaptation of a training program to the changing abilities of the patient during rehabilitation is beneficial, and it suggests that active participation is also an important factor in robot-aided therapy. Despite this, the contribution of individual therapy modalities to restoration of hemiparetic arm function remains ambiguous and an optimal application could not be discerned from the systematic analysis of existing literature (chapter 2). This stresses the need for an increased understanding of the mechanisms of recovery influenced by the various therapy modalities.

\section{Gravity compensation}

Besides passive, active-assisted and active-resisted modalities, most robotic devices incorporate arm support, or gravity compensation, in their design. This basic feature is commonly not regarded as a separate therapy modality of the device and is therefore not controlled as a part of the exercise protocol. Research has shown that the sole application of arm support influences arm movements. ${ }^{8}$ This indicates that the influence of rehabilitation robotics on improvement of arm function is not only determined by the amount of assistance applied by the robot in the direction of movement, but even support of the arm against gravity may contribute to the effect of robot-aided therapy. Therefore, gravity compensation has served as the starting point for subsequent experiments into underlying mechanisms of separate therapy modalities of rehabilitation robotics. 


\section{Influence of gravity compensation on movement execution}

Shoulder elevation and elbow flexion are often coupled in chronic stroke patients, leading to involuntary restrictions in isolated movement control (i.e., abnormal synergies). ${ }^{8,9}$ When the amount of shoulder elevation needed to lift the arm is decreased by arm support, simultaneous elbow flexion torques are reduced, leading to a larger range of elbow extension during 2D reaching with the arm at shoulder height (planar reach). ${ }^{10-13}$ Likewise, we found an increase in range of motion during natural reaching movements with gravity compensation in additional experiments, ${ }^{14,15}$ using the Freebal device, designed to provide gravity compensation during 3D arm movements. ${ }^{16}$ These studies provide information about the influence of gravity compensation on generation of joint torques and excursions, but it is not clear how support against gravity influences neuromuscular control of functional arm movements.

\section{Influence of gravity compensation on muscle activation}

The cross-sectional studies in chapters 3 and 4 show that the instantaneous influence of gravity compensation during functional reaching movements with a fixed amplitude involved a reduction of the activity levels of shoulder and elbow muscles in both healthy elderly (chapter 3 ) and mildly and moderately affected (Fugl-Meyer (FM) scores ranging from 33 to 60 points) sub-acute and chronic stroke patients (chapter 4), while movement performance is similar as in the unsupported condition. Additional experiments evaluating maximal reaching movements showed that even a larger range of motion during supported movements of stroke patients was accompanied by generally reduced levels of muscle activity. ${ }^{14}$ The temporal pattern of muscle activation was preserved in both healthy elderly (chapter 3) and stroke patients (chapter 4). This facilitating influence of gravity compensation may allow use of the neuromuscular capacity that is left after stroke for generation of the desired movement (e.g., reach), instead of for properly positioning the trunk and arm prior to the movement (e.g., sitting upright).

After stroke, motor planning, integration of sensorimotor information and generation and coordination of muscle activity may be reduced. ${ }^{17}$ In the light of the observed increase in range of motion with arm support, ${ }^{14}$ it was expected that in stroke patients gravity compensation would have a differential impact on various muscles. Remarkably, with gravity compensation, the level of muscle activity was reduced to similar degrees across different muscles in stroke patients (chapter 4). When comparing muscle activation in stroke patients (chapter 4) with the 
reference frame provided by healthy elderly (chapter 3), a rather similar influence of gravity compensation was observed. This is probably related to the sub-maximal nature of the functional reaching movements, which stayed below the maximal capacity of the included stroke patients, who could complete the movement task even without gravity compensation. This may have also contributed to the absence of changes in activation between various muscles, which had been expected considering the kine(ma)tic influence of arm support, ${ }^{8,11}$ particularly between shoulder and elbow muscles.

\section{Role of abnormal synergies during functional arm movements}

Before further investigating the potential of gravity compensation, abnormal coupling between shoulder and elbow muscles during reach was examined, to obtain more insight in the role of abnormal synergies during functional arm movements and how this may contribute to the influence of gravity compensation. In contrast to sub-maximal movements (chapter 4), a more demanding reaching task was performed by healthy persons and stroke patients with mild and moderate hemiparesis (FM scores ranging from 22 to 65 points), described in chapter 5. The reaching task involved specific resistance against shoulder elevation, applied by the Dampace exoskeleton robotic device, ${ }^{18}$ during upward, forward and outward maximal reach (as if placing a cup on a shelf), in order to provoke an abnormal coupling between the shoulder and elbow. Despite this, the resisted, but otherwise natural, reaching movements showed no signs of abnormal coupling in either joint rotations or muscle activation.

In a group of mildly to moderately affected stroke patients, the execution of a natural, resisted reaching movement was not substantially altered with resistance, in comparison to healthy persons. In addition, no abnormal coupling between the shoulder and elbow was observed in (inter-)muscle activation (chapter 5). In contrast, the studies by Beer, Dewald and colleagues do demonstrate an abnormal coupling between shoulder elevation and elbow flexion in terms of range of motion during planar reach. ${ }^{10,12,13,19}$ This discrepancy can be related to the more severely affected chronic stroke patients (i.e., FM scores as low as 15 points) and a more demanding movement task (i.e., an arm orientation of at least $75^{\circ}$ of shoulder abduction during reaching) applied in those studies. Natural reaching movements, as applied in chapters 4 and 5, do not require high levels of shoulder elevation torques. This reduces the abnormal coupling and probably limits the interference with exercises resembling activities of daily living, especially in less severely affected stroke patients. These considerations suggest that other mechanisms than 
abnormal synergies may be involved in the influence of gravity compensation on functional reaching movements.

\section{Changes in muscle activation after gravity compensation training}

Although the role of abnormal synergies during functional reach of stroke patients and the relation to gravity compensation remains ambiguous, the instantaneous influence of gravity compensation proposes a potential for improving unsupported reach. The intensive and repetitive stimulation of neuromuscular activation, facilitated instantaneously by gravity compensation (chapter 4), may transfer to improved unsupported movements when applied for a longer period. Indeed, after 6 weeks of gravity compensation training improvements in unsupported reaching distance were observed in chronic stroke patients displaying mild, moderate and severe hemiparesis (FM scores ranging from 7 to 61 points), accompanied by increased shoulder elevation throughout the reaching movement and a larger range of elbow extension (chapter 6). Sanchez et al. and Housman et al. also observed increases in unsupported reaching distance after arm support training. ${ }^{20,21}$ Although this can indicate improved isolated movement control of the shoulder and elbow, the findings of chapter 5, showing no interference of abnormal coupling during natural reaching movements in mildly and moderately affected stroke patients, suggest that other mechanisms are involved.

When looking at changes in neuromuscular control after gravity compensation training, increased levels of muscle activity of the prime movers, predominantly at the elbow, were observed in the majority of the stroke patients (chapter 6). In only one third of the patients a decreased co-activation of biceps and anterior deltoid, muscles that have been shown to act together during coupled shoulder elevation and elbow flexion in isometric contractions, ${ }^{9}$ was found. Remarkably, the increased agonist activation involved not only severely affected stroke patients, but also patients with moderate and mild hemiparesis. These findings indicate that increased activation of elbow extensors seems to play an important role in improvements of functional reach after gravity compensation training.

Studies investigating altered reach performance after stroke provide support for the relevance of agonist activation. Reach performance is often limited after stroke, with respect to distance, speed, smoothness, straightness and accuracy. ${ }^{22-}$ ${ }^{25}$ Several studies suggest muscle weakness as major contributor to impaired reach performance, ${ }^{24,25}$ and also to limitations in general arm function. ${ }^{26,27}$ To a certain extent, reduced isolated movement control seems intertwined with this relation, especially considering movement tasks that strongly emphasize movement 
coordination..$^{24}$

Likewise, improvements in reach performance during recovery from stroke seem to be largely induced by improved muscle activation. A study investigating both recovery of reach performance and changes in muscle activation over the course of rehabilitation from the acute to sub-acute phase in mildly affected stroke patients found that improved reach performance was related with improved muscle onset time, increased modulation of activity and increased relative level of muscle activity, but not with reduced co-contraction. ${ }^{28}$ This is in agreement with the findings of increased prime mover activity accompanying improved reaching distance after gravity compensation training (chapter 6 ).

\section{Potential mechanisms of recovery}

Overall, the findings of chapters 4, 5 and 6 indicate that abnormal synergies do hardly affect the ability to perform functional reaching movements in less severely affected stroke patients. In addition, this implies that increased agonist activation contributed more to improvements in reach performance than decreased counteracting activation of muscles, via either agonist/antagonist co-contraction or abnormal synergistic co-activation. This implies that improvement of arm movement ability after stroke may involve restoration of degraded neural function (i.e., restitution of function), besides potential compensatory strategies that can be used to substitute lost motor function.

The specific processes involved in the observed increase in agonist activity can not be discerned from the present research applying bipolar surface EMG recordings (chapter 6 ). Both central and peripheral mechanisms may account for the changes in muscle activation after gravity compensation training. For instance, the number or size of active motor units may be increased, ${ }^{29}$ or cortical reorganization processes, such as unmasking of latent pathways or recruitment/sprouting of new neurons, ${ }^{30}$ may be stimulated. Pharmacological interventions, such as single-dose inhibition of serotonin-reuptake in the brain, have also shown to result in a generally increased muscle activation in the lower arm of chronic stroke patients, suggesting that cortical activation can be stimulated after stroke. ${ }^{31}$ To examine such neurological and neuromuscular processes, additional specific methods are required in future research, such as the use of high density surface EMG (array) electrodes for investigation of changes in motor unit behavior, ${ }^{32}$ or brain imaging techniques for examination of changes in motor cortex activation. ${ }^{33}$ 


\section{Adherence to key aspects for motor recovery}

The findings of chapters 2 and 6 indicate that not only rehabilitation robotics have the ability to improve motor control of arm movements, also the sole application of one of its basic features, gravity compensation, is promising to improve arm movement ability. When comparing robotics (chapter 2 ) and gravity compensation (chapter 6), improvements in arm movement ability after gravity compensation training (FM score +3.3 points) are similar to improvements after robot-aided therapy (FM score +3.7 points). However, adherence to the key aspects (i.e., active and functional exercises at a high intensity), ${ }^{3,34,35}$ may differ to some extent between both interventions.

\section{Active participation}

Rehabilitation robotics involves not only active execution of movements, but also passive movements, where the robotic device moves the arm of the patient. In contrast, gravity compensation involves only active initiation and execution of movements. Since improvements in arm movement ability are similar after both interventions (chapters 2 and 6), active participation is postulated as essential component in stimulating motor recovery after stroke, whereas providing assistance to complete the movement passively (in passive and active-assisted modes) seems less crucial. This is in line with a study investigating differences in reach performance of stroke patients after equal intensity reach training with and without robotic assistance to complete the movement, in which no advantage of active-assisted training was found over free reach training. ${ }^{36}$ These findings are in agreement with the importance of active participation in stimulating restoration of arm function in exercise therapy in general.

\section{Task-specificity}

Gravity compensation applied by the custom designed Freebal device ${ }^{16}$ allows natural, 3D reaching movements strongly resembling functional use of the proximal arm (chapters 3, 4 and 6). Some robotic devices (e.g., MIT-Manus, MIME) restrict reaching movements to two dimensions. A study comparing robotaided therapy (involving 3D reaching movements) with equal intensity arm support training via sling suspension (including mainly abstract shoulder and elbow movements) revealed no substantial differences. ${ }^{37}$ Both interventions appear to be equally suitable to deliver task-specific training. The extent of task-specificity merely depends on the way the device is implemented during training. 


\section{Training intensity}

One of the principal advantages of robot-aided therapy has been stated as enabling intensive training, alleviating manual assistance by therapists. ${ }^{38}$ The intensity applied in the clinical studies investigating robot-aided therapy has generally been higher than the intensity of arm support training. Nevertheless, improvements in arm movement ability after robot-aided therapy (chapter 2) were not substantially larger than those after gravity compensation (chapter 6). The only study comparing robot-aided therapy with arm support training via sling suspension with equal training intensities revealed no significant difference in improvements in arm movement ability between both groups. Remarkably, a trend was observed that favored the sling suspension group. ${ }^{37}$ This implies that there is no advantage of robotic devices over simple arm support when considering equal training intensities.

Both gravity compensation training and robot-aided therapy have the potential for improving hemiparetic arm function. Careful interpretation of available literature indicates that gravity compensation may comply even better with the key aspect concerning active participation than robot-aided therapy. Considering these issues, the low-tech, less complex, and consequently less expensive, approach of gravity compensation would probably be more suitable for application in clinical practice than high-tech devices.

\section{Technologically supported versus conventional therapy}

When comparing rehabilitation robotics with conventional therapy, a subtle advantage of robot-aided therapy over conventional therapy has been reported, although these differences are not striking (chapter 2). For gravity compensation training alone, such comparison is not available yet, but it is expected from comparisons with robot-aided therapy that its effect will be at least as large as that of conventional therapy. A first indication of this is provided by a study comparing arm support training using an exoskeleton with elastic bands (T-WREX) to table top exercises, which revealed more or less equal improvements in reach performance and arm movement ability of the proximal arm. ${ }^{21}$ It should be noted, however, that table top exercises also involve some type of arm support, and are probably not representative of the wide array of approaches generally applied in conventional therapy, emphasizing the need for controlled studies in future research examining the application of arm support.

Considering the similarity in effect of technologically supported interventions and conventional therapy, the major advantage of technologically supported 
interventions is currently the potential to automate therapy. The application of rehabilitation devices allows one therapist to attend to multiple patients, practicing intensively, actively and functionally, at the same time. This would increase productivity and alleviate the physical burden on the therapist, which in turn can relieve the pressure on today's healthcare system, where an aging population will result in less therapists and more patients.

\section{Towards functional independence after stroke}

Although the potential to automate therapy, thereby increasing productivity, is highly valuable, the ultimate goal of stroke rehabilitation is improving functional use of the hemiparetic arm in order to promote functional independence. However, a limited transfer of training effects on motor control level to improved functional abilities has been observed for many exercise therapy interventions in stroke rehabilitation. ${ }^{40} \mathrm{~A}$ comparable predicament is observed in chapters 2 and 6 concerning robot-aided therapy and gravity compensation training. FM scores should increase at least 6.6 points (10\% of maximal score for the upper extremity part of FM) to be clinically relevant, ${ }^{41}$ which is only achieved on individual basis (chapter 6).

An example of an intervention that is promising to improve functional abilities, is constrained-induced movement therapy (CIMT), in which the unaffected arm is restrained by a mitt or sling, forcing use of the affected arm during daily life, in combination with an extensive physical therapy program focusing on activities of daily life. ${ }^{42}$ It has been suggested that the critical factor of CIMT for arm function improvement is repeated practice of activities of daily life, ${ }^{43}$ which is in line with the key aspects of intensity and task-specificity said to stimulate restoration of arm function. ${ }^{3,34,35}$ A drawback of CIMT is that only patients with a considerable level of arm function (at least $20^{\circ}$ wrist and $10^{\circ}$ finger extension) are eligible for treatment. With the use of robotics, or even the sole application of arm support, repetitive, task-specific training programs can be designed to suit also more severely affected stroke patients, who experience the largest limitations in functional use of the arm.

In order to improve functional use of the arm, current interventions can be expanded to adhere even better to the key aspects for stimulation of restoration of arm function. Addition of augmented feedback to exercises can stimulate the learning process, by making patients more aware of their performance. ${ }^{44}$ Most robot-aided therapy programs involve display of hand position and movement goals on a computer screen, informing patients about their performance by 
augmented visual feedback. The arm support application in the studies using TWREX ${ }^{20,21}$ and the study in chapter 6 also involved a virtual gaming environment providing augmented feedback. Remarkably, the results of a comparison of a group performing table top reaching exercises and a group practicing reaching with equal intensity in virtual environments providing augmented feedback showed no differences in improvements of reach and arm movement ability. ${ }^{21}$ Recent studies provide some insight in the optimal application of augmented feedback. A combination of augmented visual and sensory feedback is promising, ${ }^{45}$ as well as placing emphasis on movement errors to stimulate motor (re)learning. ${ }^{46,47}$ Since augmented feedback is closely related with the application of rehabilitation robotics, more extensive studies are needed to identify the added value and optimal way of applying augmented feedback to complement technologically supported interventions.

To maximize independent use of the arm, not only upper arm function is important, also functional use of the wrist and hand is essential. Contemporary robot-aided therapy focuses mainly on the proximal arm, and results in improvements in the proximal arm only, without generalization to the wrist and hand (chapter 2). Several technological interventions have been developed for distal arm training, such as the Bi-Manu-Track, which showed promising results. ${ }^{48}$ In addition, a distal trainer has been designed to complement the MIT-Manus robotic device for the proximal arm. ${ }^{49}$ The first preliminary results of stand-alone use of this wrist module are positive. ${ }^{50}$ However, information about the effectiveness or optimal application of simultaneous proximal and distal training using robotics is scarce.

As has also been recognized by others, ${ }^{51,52}$ future research into technological interventions should be extended to combinations of proximal and distal arm training to maximize functional use of the hemiparetic arm in activities of daily living. Of particular interest for future studies are hybrid therapeutic systems, incorporating robotics for the proximal arm, electrical stimulation for the wrist and hand, and virtual reality to provide task-specific training environments complemented with augmented feedback, in order to maximize functional independence of stroke survivors.

\section{Conclusions}

The research in this $\mathrm{PhD}$ thesis indicates that both rehabilitation robotics (chapter 2 ) and the sole application of one of its basic features, gravity compensation (chapters 4 and 6), have the ability to improve arm movement ability after stroke. 
The instantaneously facilitating influence of gravity compensation (chapters 3 and 4) is translated to unsupported reaching movements when applied during multiple training sessions (chapter 6), plausibly through intensive and repetitive stimulation of neuromuscular activation. Our findings further indicate that abnormal synergies hardly affect the ability to perform functional reaching movements in less severely affected stroke patients (chapter 5). Instead, more pronounced activation of prime movers is mainly involved in improvements of functional reach (chapter 6). This implies that improvement of arm movement ability after stroke may involve restoration of degraded neural function (i.e., restitution of function), besides potential compensatory strategies used to substitute lost motor function. Moreover, interventions aimed at improvement of functional reach should mainly target the (in)ability to activate prime movers, instead of focusing on reducing abnormal coupling between the shoulder and elbow.

The potential to improve arm movement ability after stroke does currently not differ much between robot-aided therapy, gravity compensation training and conventional therapy. As advantage over conventional therapy, both robot-aided therapy and gravity compensation training allow automation of therapy, thereby offering the possibility to increase productivity, and reduce costs, of the healthcare system. Considering implementation in clinical practice, the low-tech application of gravity compensation may be more suitable than high-tech devices, since the effects seem to be comparable between both interventions, and gravity compensation may encourage active participation even more than robot-aided therapy. Improvement of arm movement ability may be stimulated further by including augmented feedback into current technological interventions, in a way complying as much as possible with the key aspects for motor recovery. In addition, not only the proximal arm should be targeted, also the wrist and hand should be involved in functional training for optimal results. In particular, hybrid therapeutic systems, incorporating robotics, electrical stimulation and virtual reality, are promising topics for future research, in order to maximize functional independence after stroke. 


\section{References}

1. Rosamond W, Flegal K, Furie K, et al. Heart disease and stroke statistics 2008 update. Circulation 2008;117:e25-e146

2. Kwakkel G, Kollen BJ, Van der Grond J, Prevo AJH. Probability of regaining dexterity in the flaccid upper limb: impact of severity of paresis and time since onset in acute stroke. Stroke 2003;34:2181-2186

3. Kwakkel G, Wagenaar RC, Twisk JWR, Lankhorst GJ, Koetsier JC. Intensity of leg and arm training after primary middle-cerebral-artery stroke: a randomised trial. Lancet 1999;354:189-194

4. Kwakkel G, Kollen B, Lindeman E. Understanding the pattern of functional recovery after stroke: Facts and theories. Restor Neurol Neurosci 2004;22:281-299

5. Krebs HI, Hogan N, Aisen ML, Volpe BT. Robot-aided neurorehabilitation. IEEE Trans Rehabil Eng 1998;6(1):75-87

6. Stein J, Krebs HI, Frontera WR, Fasoli SE, Hughes R, Hogan N. Comparison of two techniques of robot-aided upper limb exercise training after stroke. Am J Phys Med Rehabil 2004;83(9):720-728

7. Fasoli SE, Krebs HI, Stein J, Frontera WR, Hogan N. Effects of robotic therapy on motor impairment and recovery in chronic stroke. Arch Phys Med Rehabil 2003; 84(4):477-482

8. Beer RF, Given JD, Dewald JPA. Task-dependent weakness at the elbow in patients with hemiparesis. Arch Phys Med Rehabil 1999;80:766-772

9. Dewald JPA, Pope PS, Given JD, Buchanan TS, Rymer WZ. Abnormal muscle coactivation patterns during isometric torque generation at the elbow and shoulder in hemiparetic subjects. Brain 1995;118:495-510

10. Beer RF, Dewald JPA, Rymer WZ. Deficits in the coordinaton of multijoint arm movements in patients with hemiparesis: evidence for disturbed control of limb dynamics. Exp Brain Res 2000;131:305-319

11. Dewald JPA, Beer RF. Abnormal joint torque patterns in the paretic upper limb of subjects with hemiparesis. Muscle Nerve 2001;24:273-283

12. Beer RF, Dewald JPA, Dawson ML, Rymer WZ. Target-dependent differences between free and constrained arm movements in chronic hemiparesis. Exp Brain Res 2004; $156: 458-470$

13. Beer RF, Ellis MD, Holubar BG, Dewald JPA. Impact of gravity loading on post-stroke reaching and its relationship to weakness. Muscle Nerve 2007;36:242-250

14. Prange GB, Stienen AHA, Jannink MJA, Van der Kooij H, IJzerman MJ, and Hermens $\mathrm{HJ}$. Increased range of motion and decreased muscle activity during maximal reach with gravity compensation in stroke patients. In: Proceedings of the 10th International Conference on Rehabilitation Robotics (ICORR); June 13-15, 2007, Noordwijk aan Zee, the Netherlands:467-471 
15. Stienen AHA, Van der Helm FCT, Prange GB, Jannink MJA, and Van der Kooij $H$. Effects of gravity compensation on the range-of-motion of the upper extremities in robotic rehabilitation after stroke. In: Proc Inter Shoulder Group; 2006 Oct 9-10, Chicago (IL), USA

16. Stienen AHA, Hekman EEG, Van der Helm FCT, Prange GB, Jannink MJA, Aalsma AMM, and Van der Kooij H. Freebal: dedicated gravity compensation for het upper extremities. In: Proceedings of the 10th International Conference on Rehabilitation Robotics (ICORR); June 13-15, 2007, Noordwijk aan Zee, the Netherlands:804-808

17. Chae J, Yang G, Park BK, Labatia I. Muscle weakness and cocontraction in upper limb hemiparesis: relationship to motor impairment and physical disability. Neurorehabil Neural Repair 2002;16:241-248

18. Stienen AHA, Hekman EEG, Van der Helm FCT, Prange GB, Jannink MJA, Aalsma $A M M$, and Van der Kooij H. Dampace: dynamic force-coordination trainer for the upper extremities. In: Proceedings of the 10th International Conference on Rehabilitation Robotics (ICORR); June 13-15, 2007, Noordwijk aan Zee, the Netherlands:820-826

19. Dewald JPA, Sheshadri V, Dawson ML, Beer RF. Upper-limb discoordination in hemiparetic stroke: implications for neurorehabilitation. Top Stroke Rehabil $2001 ; 8(1): 1-12$

20. Sanchez RJ, Liu J, Rao S, et al. Automating arm movement training following severe stroke: functional exercises with quantitative feedback in a gravity-reduced environment. IEEE Trans Neural Syst Rehabil Eng 2006;14(3):378-389

21. Housman SJ, Scott KM, Reinkensmeyer DJ. A randomized controlled trial of gravitysupported, computer-enhanced arm exercise for individuals with severe hemiparesis. Neurorehabil Neural Repair 2009;23(5):505-514

22. Kamper DG, McKenna-Cole AN, Kahn LE, Reinkensmeyer DJ. Alterations in reaching after stroke and their relation to movement direction and impairment severity. Arch Phys Med Rehabil 2002;83:702-707

23. Reisman DS, Scholz JP. Aspects of joint coordination are preserved during pointing in persons with post-stroke hemiparesis. Brain 2003;126: 2510-2527

24. Wagner JM, Lang CE, Sahrmann SA, et al. Relationships between sensorimotor impairments and reaching deficits in acute hemiparesis. Neurorehabil Neural Repair 2006;20:406-416

25. Zackowski KM, Dromerick AW, Sahrmann SA, Thach WT, Bastian AJ. How do strength, sensation, spasticity and joint individuation relate to the reaching deficits of people with chronic hemiparesis? Brain 2004;127(5):1035-1046

26. Harris JE, Eng JJ. Paretic upper-limb strength best explains arm activity in people with stroke. Phys Ther 2007;87:88-97

27. Canning CG, Ada L, Adams R, O'Dwyer NJ. Loss of strength contributes more to physical disability after stroke than loss of dexterity. Clin Rehabil 2004;18:300-308

28. Wagner JM, Dromerick AW, Sahrmann SA, Lang CE. Upper extremity muscle 
activation during recovery of reaching in subjects with post-stroke hemiparesis. Clin Neurophysiol 2007;118:164-176

29. Kallenberg LAC, Hermens HJ. Motor unit properties of biceps brachii in chronic stroke patients assessed with high-density surface EMG. Muscle Nerve 2009;39:177-185

30. Calautti C, Baron JC. Functional neuroimaging studies of motor recovery after stroke in adults: a review. Stroke 2003;34:1553-1566

31. Van Genderen HI, Nijlant JMM, Van Putten MJAM, Movig KLL, IJzerman MJ. Single dose of fluoxetine increases muscle activation in chronic stroke patients. Clin Neuropharmacol 2008 (in press)

32. Kallenberg LAC, Hermens HJ. Behaviour of a surface EMG based measure for motor control: Motor unit action potential rate in relation to force and muscle fatigue. J Electromyogr Kinesiol 2008;18:780-788

33. Cramer SC, Bastings EP. Mapping clinically relevant plasticity after stroke. Neuropharmacology 2000;39:842-851

34. Feys HM, De Weerdt WJ, Selz BE, et al. Effect of a therapeutic intervention for the hemiplegic upper limb in the acute phase after stroke. A single-blind, randomized, controlled multicenter trial. Stroke 1998;29:785-792

35. Barreca S, Wolf SL, Fasoli S, Bohannon R. Treatment interventions for the paretic upper limb of stroke survivors: a critical review. Neurorehabil Neural Repair 2003; 17:220-226

36. Kahn LE, Zygman ML, Rymer WZ, Reinkensmeyer DJ. Robot-assisted reaching exercise promotes arm movement recovery in chronic hemiparetic stroke: a randomized controlled pilot study. J NeuroEng Rehabil 2006;3:12

37. Amirabdollahian F, Loureiro R, Gradwell E, Collin C, Harwin W, Johnson G. Multivariate analysis of the Fugl-Meyer outcome measures assessing the effectiveness of GENTLE/S robot-mediated stroke therapy. ] NeuroEng Rehabil 2007;4:4

38. Krebs HI, Hogan N, Volpe BT, Aisen ML, Edelstein L, Diels C. Overview of clinical trials with MIT-MANUS: a robot-aided neuro-rehabilitation facility. Technol Health Care $1999 ; 7(6): 419-423$

39. Krebs HI, Volpe BT, Aisen ML, Hogan N. Increasing productivity and quality of care: robot-aided neuro-rehabilitation. J Rehabil Res Dev 2000; 37(6):639-652.

40. Wagenaar RC, Meijer OG. Effects of stroke rehabilitation: a critical review of the literature. J Rehabil Sci 1991;4(3):61-73

41. Gladstone DJ, Danells CJ, Black SE. The Fugl-Meyer assessment after stroke: a critical review of its measurement properties. Neurorehab Neural Repair 2002;16(3):232-240

42. Wolf SL, Blanton S, Baer H, Breshears J, Butler AJ. Repetitive task practice: A critical review of constraint-induced movement therapy in stroke. Neurol 2002;8:325-338

43. Page SJ, Sisto SA, Levine $P$, Johnston MV, Hughes $M$. Modified constrainded induced therapy: A randomized feasibility and efficacy study. J Rehabil Res Dev 2001;38(5):583-590 
44. Winstein $\mathrm{C}$, Campbell Stewart J. Conditions of task practice for individuals with neurologic impairments. In: Selzer M, Clarke S, Cohen L, Duncan P, Gage F, eds. Textbook of neural repair and rehabilitation. Medical Neurorehabilitation Volume II; Cambridge, UK: Cambridge University Press; 2006: 89-102

45. Molier BI, Van Asseldonk EHF, Hermens HJ, Jannink MJA. Nature, timing, frequency, and type of augmented feedback; does it influence motor relearning of the hemiparetic arm? A systematic review. (submitted 2009)

46. Van Asseldonk EHF, Wessels M, Stienen AHA, Van der Helm FCT, Van der Kooij H. Influence of haptic guidance in learning a novel visuomotor task. J Physiol Paris 2009 (in press)

47. Patton JL, Mussa-Ivaldi FA. Robot-assisted adaptive training: Custom force fields for teaching movement patterns. IEEE Trans Biomed Eng 2004;51(4):636-646

48. Hesse S, Schulte-Tigges G, Konrad M, Bardeleben A, Werner C. Robot-assisted arm trainer for the passive and active practice of bilateral forearm and wrist movements in hemiparetic subjects. Arch Phys Med Rehabil 2003;84(6):915-920

49. Krebs HI, Celestino J, Williams D, Ferraro M, Volpe BT, Hogan N. A wrist extension to MIT-MANUS. In: Bien Z, Stefanov D, eds. Advances in human-friendly robotic technologies for movement assistance/movement restoration for people with disabilities; Springer-Verlag series lecture notes in control and information sciences. Berlin-Heidelberg: Springer-Verlag; 2004: chapter 24

50. Krebs HI, Volpe BT, Williams D, et al. Robot-aided neurorehabilitation: a robot for wrist rehabilitation. IEEE Trans Neural Syst Rehabil Eng 2007;15(3):327-335

51. Fasoli SE, Krebs HI, Hogan N. Robotic technology and stroke rehabilitation: translating research into practice. Top Stroke Rehabil 2004;11(4):11-19

52. IJzerman MJ, Renzenbrink GJ, Geurts ACH. Neuromuscular stimulation after stroke: from technology to clinical deployment. Expert Rev Neurother 2009;9(4):541-552 



\section{Summary}

A cerebrovascular accident, or stroke, is a major cause of permanent disability. The annual incidence rate of stroke in both the Netherlands and the USA is approximately 250 new cases per 100,000 persons. A stroke can lead to damage of nerve pathways between the brain and the spinal cord and to reduced integration of sensory and motor information during motor planning in the brain, limiting selective activation of muscle tissue. Regarding the motor domain, this can result in a variety of symptoms, such as muscle weakness, spasticity, and limited movement coordination, in addition to sensory, cognitive and psychological symptoms. With respect to the upper extremity, impaired arm and hand function may cause serious limitations in activities of daily living in at least $60 \%$ of stroke patients.

To identify effective therapeutic interventions in stroke rehabilitation, emphasis has been placed more and more on evidence-based physical therapy during the last decades, as well as increasing research into principles of motor relearning and processes of cortical reorganization. This has presented several key aspects that have the potential to stimulate restoration of arm function after stroke: active initiation and execution of movements, high training intensity and application of functional exercises. Technological innovations provide an opportunity to design interventions that take these key aspects into account. A promising application is the use of rehabilitation robotics to complement conventional therapy. Robotic devices can be programmed to apply forces in a smart way. In passive mode, the robotic device imposes movements by moving the arm of the patient in a preplanned trajectory. In active-assisted mode, the robotic device provides assistance to aid in completion of the movement. In active-resisted mode, the robotic device delivers resistance against movements actively executed by the patient.

These therapy modalities will stimulate the neuromuscular system to various extents. It is still unclear if the way in which robot-aided therapy is currently applied is the optimal approach to achieve maximal improvements in arm function. Maximizing functional independence of stroke patients can involve restoration of degraded neural function via stimulation of cortical reorganization, or compensatory strategies to circumvent lost motor function. Since many approaches intervene at the motor control level, muscle activation may change consequently. By investigating changes in muscle activation due to application of robotic devices, the merits of different approaches may be discerned. Such information can also aid 
in understanding which mechanisms of recovery are targeted by the application of rehabilitation robotics. This knowledge can then be used to determine how to take advantage of the merits of rehabilitation robotics Therefore, the main objective of the research reported in this $\mathrm{PhD}$ thesis is to obtain a better understanding of the impact of different therapy modalities of rehabilitation robotics on neuromuscular control of arm movements of stroke patients.

A systematic review of existing literature (chapter 2) shows that robot-aided therapy is able to improve motor control of the trained, proximal arm of both subacute and chronic stroke patients. Although the findings indicate that the application of rehabilitation robotics is promising, perhaps even more so than conventional therapy, the most effective application and its underlying mechanisms could not be discerned. Besides passive, active-assisted and active-resisted modalities, most robotic devices incorporate arm support in their design. This basic feature is commonly not regarded as a separate therapy modality of the device, and is therefore not controlled as a part of the exercise protocol. Research has shown that the sole application of arm support influences arm movements. This indicates that, besides the amount of assistance applied by the robot in the direction of movement, only support of the arm against gravity may already contribute to the effect of robot-aided therapy. Therefore, gravity compensation has served as the starting point for subsequent experiments.

Shoulder elevation and elbow flexion are often coupled in chronic stroke patients, leading to involuntary restrictions in isolated movement control (i.e., abnormal coupling). When the amount of shoulder elevation needed to lift the arm is decreased by arm support, simultaneous elbow flexion torques are reduced, leading to a larger range of elbow extension during 2D supported reaching with the arm at shoulder height. However, from this research is not clear which neurophysiological mechanisms are involved, especially considering natural reaching movements resembling activities of daily life. The cross-sectional experiments described in chapters 3 and 4 show that the sole application of gravity compensation influenced neuromuscular control of functional arm movements. With gravity compensation, muscle activity levels during reach with a fixed amplitude were reduced across muscles in both healthy elderly (chapter 3 ) and mildly and moderately affected stroke patients (chapter 4). Movement performance was similar in both conditions, and the temporal pattern of muscle activity was also preserved in both healthy elderly and stroke patients. Remarkably, no signs of a reduction in abnormal coupling between shoulder and elbow muscles were observed. Even when adding resistance to shoulder elevation during upward reach 
of mildly and moderately affected stroke patients, no indication of such abnormal synergies in either joint rotations or muscle activation was found (chapter 5). This is likely to be related to a combination of the severity of hemiparesis and the submaximal nature of the movement tasks, resembling activities of daily living, requiring only limited shoulder elevation torques.

Despite the ambiguity about the role of abnormal synergies during functional reach and its relation with gravity compensation, the instantaneous facilitating influence of gravity compensation in mildly and moderately affected stroke patients (chapter 4) proposes a potential benefit of a longer-term application. Intensive stimulation of neuromuscular activation during supported movements might induce improvements in unsupported reach. Therefore, a study applying gravity compensation as training intervention was performed (chapter 6 ). After 6 weeks of reach training with gravity compensation, improved unsupported reaching was accompanied by increased activation of agonists, mainly at the elbow, in the majority of mildly, moderately, and severely affected chronic stroke patients. This indicates that a more pronounced activation of prime movers was mainly involved in improvements of sub-maximal, natural reaching movements. This implies that restoration of arm movement ability after stroke may involve restoration of degraded neural function (i.e., restitution of function), besides potential compensatory strategies used to substitute lost motor function. Accordingly, interventions aimed at improvement of functional reach should mainly target the (in)ability to activate prime movers, instead of focusing on reducing abnormal coupling between the shoulder and elbow.

The potential to improve arm movement ability after stroke does currently not differ much between robot-aided therapy, gravity compensation training and conventional therapy. As advantage over conventional therapy, both robot-aided therapy and gravity compensation training allow automation of therapy, thereby offering the possibility to increase productivity, and reduce costs, of the healthcare system. Considering implementation in clinical practice, the low-tech application of gravity compensation may be more suitable than high-tech devices, since the effects seem to be comparable between both interventions, and gravity compensation may encourage active participation even better than robot-aided therapy. Restoration of arm function may be stimulated further by including augmented feedback into current technological interventions, in a way complying as much as possible with the key aspects for motor recovery. In addition, not only the proximal arm should be targeted, also the wrist and hand should be involved in functional training for optimal results. In particular, hybrid therapeutic systems, 
incorporating robotics, neuromuscular stimulation and virtual reality, are promising topics for future research, in order to maximize functional independence after stroke. 


\section{Samenvatting}

Een cerebrovasculair accident, ook wel CVA of beroerte, is een van de belangrijkste oorzaken van permanente invaliditeit. De jaarlijkse incidentie van CVA is ongeveer 250 nieuwe gevallen per 100.000 personen in zowel Nederland als in de Verenigde Staten. Een CVA kan leiden tot beschadiging van de zenuwbanen van de hersenen naar het ruggenmerg en tot een verminderde integratie van sensorische en motorische informatie tijdens het plannen van motorische taken, wat een selectieve activatie van spierweefsel bemoeilijkt. Op motorisch gebied kan dit resulteren in symptomen zoals spierzwakte, spasticiteit en een verminderde coördinatie van bewegingen, naast sensorische, cognitieve en psychologische symptomen. Met betrekking tot de bovenste extremiteit veroorzaakt een beperkte arm- en handfunctie in ten minste $60 \%$ van de CVA-patiënten aanzienlijke beperkingen in activiteiten van het dagelijks leven.

Om effectieve therapeutische interventies voor CVA-revalidatie te identificeren, is de laatste decennia in toenemende mate nadruk gelegd op 'evidence-based' fysiotherapie (gebaseerd op wetenschappelijk bewijs), naast een toenemende mate van onderzoek naar principes van motorisch herleren en processen van corticale reorganisatie. Dit heeft geleid tot een aantal sleutelaspecten, die het mogelijk maken om herstel van de armfunctie na een CVA te stimuleren: actieve initiatie en uitvoering van bewegingen, hoge trainingsintensiteit en toepassing van functionele oefeningen. Door technologische innovaties kunnen specifieke interventies ontworpen worden met het oog op deze sleutelaspecten. Een veelbelovende toepassing is het gebruik van revalidatierobots als aanvulling op conventionele therapie. Robotische apparaten kunnen worden geprogrammeerd om op een slimme manier kracht te leveren. In passieve modus legt het robotische apparaat een beweging op door de arm van de patiënt langs een van tevoren gepland traject te leiden. In actief-assistieve modus biedt de robot assistentie om de beweging te voltooien. In actief-resistieve modus geeft de robot weerstand tegen de beweging die de patiënt actief uitvoert.

Deze verschillende therapiemodaliteiten stimuleren het neuromusculaire systeem in meer of mindere mate. Het is nog onbekend of de manier waarop robotgeassisteerde therapie heden ten dage wordt toegepast de optimale benadering is om maximale verbeteringen in armfunctie te bewerkstelligen. Het maximaliseren van de functionele onafhankelijkheid na een CVA kan zowel via herstel van verminderde neurale functie (stimulering van corticale reorganisatie), alsook door 
toepassing van compensatiestrategieën om de verloren gegane functie te passeren. Veel interventies richten zich op het niveau van de neuromusculaire aansturing, wat tot veranderingen in de spieractivatie kan leiden. Door veranderingen in spieractivatie naar aanleiding van de toepassing van robotische apparaten te evalueren,kunnen wellicht de waardevolle aspecten van verschillende robotische benaderingen worden bepaald. Zulke informatie kan ook helpen te begrijpen op welke mechanismen van herstel de toepassing van robotische apparaten aangrijpt. Deze inzichten kunnen dan worden aangewend om effectieve toepassingen van robotische apparaten te identificeren en om te bepalen hoe de meest waardevolle aspecten kunnen worden ingezet in de revalidatie, zodat optimale resultaten kunnen worden behaald. Het doel van het onderzoek in dit proefschrift is dan ook het verkrijgen van een beter begrip van de invloed van verschillende therapiemodaliteiten van revalidatierobots op de neuromusculaire aansturing van armbewegingen van CVA-patiënten.

Een systematisch overzicht van de bestaande literatuur (hoofdstuk 2) laat zien dat robot-geassisteerde therapie de motorische aansturing van de getrainde proximale arm van zowel sub-acute als chronische CVA-patiënten kan verbeteren. Alhoewel deze bevindingen aangeven dat de toepassing van revalidatierobots veelbelovend is, misschien zelfs beter dan conventionele therapie, kunnen de meest effectieve toepassingen en de onderliggende mechanismen niet worden onderscheiden. Naast passieve, actief-assistieve en actief-resistieve modaliteiten integreren de meeste robotische apparaten armondersteuning in hun ontwerp. Dit basale aspect wordt vaak niet beschouwd als een opzichzelfstaande therapiemodaliteit van het apparaat en wordt dan ook niet gecontroleerd als onderdeel van het therapieprotocol. Onderzoek heeft aangetoond dat alleen de toepassing van armondersteuning al in staat is om armbewegingen te beïnvloeden. Dit wijst erop dat, naast de mate van assistentie door de robot, alleen ondersteuning van de arm tegen de zwaartekracht al een bijdrage kan leveren aan het effect van robot-geassisteerde therapie. Derhalve wordt zwaartekracht compensatie als uitgangspunt genomen voor volgende experimenten.

Schouderelevatie en elleboogflexie zijn vaak gekoppeld in chronische CVApatiënten, wat kan leiden tot onwillekeurige belemmeringen in een geïsoleerde bewegingsaansturing, ook wel abnormale koppeling genoemd. Wanneer de hoeveelheid schouderelevatie benodigd om de arm op te tillen verminderd wordt door armondersteuning, neemt de gelijktijdige, gekoppelde elleboogflexie af, wat resulteert in een grotere elleboogextensie tijdens 2D reikbewegingen, waarbij de arm op schouderhoogte wordt gehouden. Echter, uit dit onderzoek wordt niet 
duidelijk welke neurofysiologische mechanismen betrokken zijn, vooral in het geval van natuurlijke reikbewegingen die lijken op activiteiten in het dagelijks leven. De cross-sectionele experimenten beschreven in hoofdstukken 3 en 4 geven aan dat alleen de toepassing van zwaartekrachtcompensatie al de neuromusculaire aansturing van functionele armbewegingen beïnvloedt. Met zwaartekracht compensatie nam de mate van spieractivatie van meerdere spieren tijdens reikbewegingen met een vaste amplitude af in zowel gezonde ouderen (hoofdstuk 3), als mild tot matig aangedane CVA-patiënten (hoofdstuk 4). De bewegingsuitvoering was vergelijkbaar in beide condities en ook het patroon van spieractivatie was onveranderd in zowel gezonde ouderen als CVA-patiënten. Daarnaast is het opmerkelijk dat geen aanwijzingen van een verminderde abnormale koppeling tussen de schouder en elleboog zijn gevonden. Zelfs wanneer weerstand tegen schouderelevatie wordt gegeven tijdens opwaarts reiken door mild en matig aangedane CVA-patiënten, wees niets op de aanwezigheid van zo'n abnormale koppeling (hoofdstuk 5). Dit is waarschijnlijk gerelateerd aan een combinatie van de mate van aangedaanheid na de CVA en de submaximale aard van de bewegingstaken, die activiteiten van het dagelijks leven weerspiegelden, zodat slechts een beperkt schouderelevatie-moment gegenereerd hoefde te worden.

Ondanks dat de rol van abnormale koppeling tijdens functionele reikbewegingen, en de relatie met zwaartekrachtcompensatie, nog onduidelijk is, geeft de instantane faciliterende invloed van zwaartekrachtcompensatie in mild tot matig aangedane CVA-patiënten (hoofdstuk 4) aanwijzingen voor een mogelijk voordeel van toepassing op de langere termijn. Intensieve stimulatie van neuromusculaire activatie tijdens bewegingen met ondersteuning zou verbeteringen in reiken zonder ondersteuning kunnen veroorzaken. Dit gaf aanleiding om een studie uit te voeren waarin zwaartekrachtcompensatie als trainingsinterventie wordt toegepast (hoofdstuk 6). Na 6 weken reiktraining met zwaartekrachtcompensatie werd een verbeterd reiken zonder ondersteuning vergezeld van een toegenomen activatie van agonisten, vooral rond de elleboog, in de meerderheid van de mild, matig en ernstig aangedane, chronische CVA-patiënten. Dit wijst erop dat een meer nadrukkelijke activatie van agonisten een prominente rol speelt bij de verbetering van submaximale, natuurlijke reikbewegingen. Dit impliceert herstel van de verminderde neurale functie bij de verbetering van de armvaardigheid, naast een mogelijke rol van compensatiestrategieën om de verloren gegane functie te passeren. Volgens deze bevindingen zouden interventies voor het verbeteren van functioneel reiken dan ook met name gericht moeten zijn op het (on)vermogen om 
agonisten te activeren, in plaats van het verminderen van een abnormale koppeling tussen de schouder en elleboog.

Ter verbetering van de armvaardigheid na een CVA doen op dit moment robotgeassisteerde therapie, zwaartekrachtcompensatie-training en conventionele revalidatie niet voor elkaar onder. Een voordeel van robot-geassisteerde therapie en zwaartekrachtcompensatie-training boven conventionele revalidatie is de mogelijkheid om de behandeling te automatiseren (een therapeut kan meerdere patiënten tegelijk behandelen), zodat de productiviteit van de gezondheidszorg kan toenemen en de kosten kunnen worden gereduceerd. Aangezien de effecten van deze beide interventies vergelijkbaar zijn, lijkt de 'low-tech' toepassing van zwaarte-krachtcompensatie meer geschikt voor implementatie in de praktijk dan 'high-tech' apparaten. Daarnaast is het aannemelijk dat zwaartekrachtcompensatie het actief inzetten van de arm tijdens training meer bevordert dan robotische apparaten dat doen. Herstel van armfunctie kan verder worden gestimuleerd door 'augmented' (extrinsieke) feedback toe te voegen aan bestaande technologische interventies, op zo'n manier dat zoveel mogelijk rekening gehouden wordt met de sleutelaspecten die motorisch herleren kunnen stimuleren. Verder is het essentieel om niet alleen de proximale arm te trainen, maar ook de pols en hand te betrekken in functionele training om optimale resultaten te kunnen bereiken. Speciale belangstelling voor toekomstig onderzoek gaat uit naar hybride therapeutische systemen, waarin robotica, neuromusculaire stimulatie en virtuele omgevingen worden gecombineerd om het herstel van de arm te maximaliseren, zodat CVA-patiënten een zo groot mogelijke onafhankelijkheid in het dagelijks leven kunnen behalen. 


\section{Dankwoord}

Het zit erop, het promotieonderzoek. Het boekje is klaar. Maar voordat het tijd is voor ontspanning, is het tijd om een aantal mensen die een belangrijke rol hebben gespeeld tijdens mijn promotieonderzoek te bedanken.

Michiel, waardevolle inhoudelijke begeleiding en een hele fijne samenwerking gingen hand in hand. Jouw frisse blik kon me telkens weer op het goede spoor helpen en je gaf me de gelegenheid te groeien in het onderzoek. Daarnaast heb ik veel van je kunnen leren over allerlei vaardigheden die van pas komen bij het organiseren en presenteren van onderzoek, vooral ook tijdens congressen. Met tussendoor natuurlijk de nodige toeristische uitstapjes. Ik zal onze eerste, en naar het bleek een-na-laatste, internationale trip naar Vancouver, samen met Hans, niet snel vergeten! Dus ontzettend bedankt, Michiel. Door je carrièreswitch heb je de afronding van mijn promotie jammer genoeg van wat minder dichtbij meegemaakt, maar ik ben erg blij dat je er bij bent om het eindresultaat te aanschouwen en het eerste robot-project van het cluster samen af te kunnen sluiten.

Hermie, je wijze raad heeft me vaak geholpen de juiste lijn te ontdekken in de data en in manieren om de uitkomsten op papier te zetten. Natuurlijk waren ook je enorme kennis en inzicht op EMG-gebied onmisbaar. Niet alleen inhoudelijk heb ik veel aan je begeleiding gehad, ook heb je mij begeleid naar mijn eerste congres, en zelfs op mijn eerste vliegreis, naar Turijn. Aan dit alles heb ik veel bijzondere herinneringen overgehouden. Bedankt voor de fijne samenwerking.

Maarten, jij hebt vooral een sturende rol gehad, met name bij het opzetten van de studies en artikelen. Bij elk overleg gaf je de aanzet tot nieuwe ideeën en mogelijkheden, waardoor ik weer op het goede spoor kon belanden. Vooral op methodologisch en epidemiologisch vlak heb ik veel van je kunnen leren. Bedankt voor je waardevolle adviezen, die me telkens gestimuleerd hebben het onderzoek zo goed mogelijk vorm te geven.

Ook de leden van de commissie wil ik hartelijk bedanken voor hun bijdrage. Prof. dr. Jane Burridge, dr. Peter Schenk, prof. dr. ir. Bart Koopman, prof. dr. Hans Rietman \& prof. dr. ir. Peter Veltink, I am honoured to have you on my doctorate board, thank you all very much for your willingness to evaluate my dissertation.

Arno, jij bent op hetzelfde onderzoeksproject gepromoveerd, wat betekent dat we heel veel hebben samengewerkt, jij de technische kant met het ontwerpen van 
robotische apparaten, ik de klinische kant met het evalueren ervan. Zonder jouw ingenieuze ontwerpen en vele analyses die je voor me hebt gedaan, zou mijn promotieonderzoek er heel anders hebben uitgezien. Ook al hebben we verschillende achtergronden, wat zo nu en dan tot komische spraakverwarringen leidde (actief versus passief?), hebben we onze draai in het project goed kunnen vinden en heb ik prettig met je samengewerkt. Bedankt voor de inzet en toewijding waarmee je je essentiële rol in het project hebt vervuld.

Herman, Frans, Gert \& Arthur, zonder jullie als projectpartners zou ik heel wat minder te evalueren hebben gehad. Bedankt voor jullie inbreng in dit project tijdens de vele productieve en ook gezellige projectgroepvergaderingen.

Leendert, je lab-technische advies over EMG-apparatuur en robotische apparaten zorgde voor een soepel verloop van mijn studies. Ook heb je vaak willen helpen met allerlei andere zaken, zoals analyses en afbeeldingen. Je hebt meer dan eens gezorgd dat ik een deadline kon halen, mét goede figuren. Bedankt!

Jos, Wil, Rik, Karin, Joke, Marjan, Inger \& Gerda, bedankt voor alle ondersteuning op (computer)technisch, statistisch en organisatorisch vlak. Jullie hulp en vele tips hebben, direct en indirect, veel bijgedragen aan de totstandkoming van dit boekje.

Al dit onderzoek had echter niet kunnen worden gedaan zonder de onbaatzuchtige inzet van alle deelnemers aan de studies. Ook hebben veel artsen, therapeuten en ondersteunend personeel uit de kliniek mij geholpen met de praktische uitvoering van de studies en het werven van deelnemers. Hartelijk dank voor jullie hulp.

In het bijzonder Bertjo \& Jacintha hebben een grote rol gespeeld. Bedankt voor het in het juiste perspectief plaatsen van de opzet van metingen en van de resultaten vanuit jullie klinische expertise als revalidatiearts en fysiotherapeut. En niet in het minst wil ik jullie hartelijk danken voor de hulp met de werving van patiënten voor deelname aan de studies en voor de prettige samenwerking.

Jaap, ook uit jouw klinisch-wetenschappelijke ervaring heb ik kunnen putten, bedankt. Ik kijk ernaar uit om meer met je samen te werken in de komende jaren, in je nieuwe functie als clustermanager.

Vooral ook de gezelligheid, belangstelling en goede sfeer onder alle collega's bij RRD dragen veel bij aan het werkplezier, iedereen bedankt! Laura, Janine, Rianne, Anke, Hanneke, Judith, Jasper, Thijs, Birgit, Marieke, Corien \& Karlijn, bedankt ook voor het kunnen delen van de goede nieuwtjes, maar ook de frustraties, die langskomen tijdens zo'n promotieonderzoek. 
Laura \& Thijs, jullie hebben daarnaast ook een grote inhoudelijke rol gespeeld in mijn promotieonderzoek. Laura, bedankt voor je uitgebreide ondersteuning bij de EMG-analyses en alles wat ik op EMG-gebied van je heb kunnen leren. Daarnaast heb je me vaak geholpen met handige tips over het organiseren van onderzoek en promotie, helemaal in de rol van paranimf. Bedankt voor dit alles. Thijs, zonder je onvermoeibare inzet voor de trainingsstudie, jouw afstudeeropdracht, zou die studie lang niet zo goed van de grond zijn gekomen en zo'n motiverende trainingsopstelling hebben! En door je geduldige uitleg van technische zaken heb ik heel wat opgestoken op dat vlak. Bedankt voor de fijne samenwerking.

En Karlijn, als mijn kamergenootje heb je veel verhalen van me aangehoord. Een groot deel van onze gesprekken ging over de ups van het werk, of over manieren om van downs weer ups te kunnen maken. Maar het liefst ging het over allerlei andere dingen: vakantieplannen, huis- \& tuinplannen, foto's van die plannen, volleybal-, tennis-, dans-, en niet te vergeten, hardloop-belevenissen. Toch had je ook altijd een luisterend oor voor de downs. Je goede adviezen en tips, helemaal in de rol van paranimf, werkten altijd erg bemoedigend. Ontzettend bedankt!

Daarnaast ben ik heel dankbaar voor de steun van familie en vrienden buiten het werk om. Lieve Martijn, heel erg bedankt voor je steevaste vertrouwen in mijn kunnen. Jouw bemoedigende woorden, daadkrachtige adviezen en vele knuffels hebben me vaak het benodigde steuntje in de rug gegeven. Het besef dat je achter mij en mijn werk staat, betekent heel veel voor me. Jij betekent heel veel voor me. Lieve pap, mam \& sussie Harmke, bedankt dat jullie altijd voor me klaar staan. En Remco, bedankt voor de vrolijke noot die je hier (vaak letterlijk!) aan toevoegt. Ik kan altijd bij jullie terecht, met werk of niet-werk. De gezelligheid en warmte bij jullie kon telkens zorgen voor afleiding en opvrolijking wanneer dat nodig was. Heel erg bedankt voor jullie voortdurende steun, vertrouwen, aanmoediging en geloof in mij.

Lieve oma (\& opa), familie \& 'schoon'familie, bedankt voor jullie belangstelling in mijn studie en werk. Het geeft een goed gevoel te weten dat gewaardeerd wordt wat je doet. Lieve school-, studie-, dans- \& volleybalvriendinnen en -vrienden, bedankt voor jullie luisterende oren en de broodnodige lol en gezelligheid als welkome afleiding, tijdens inspanning en ontspanning!

Hiermee komt er een eind aan dit boekje, en aan mijn promotieonderzoek. Na het harde werken is er nu gelukkig weer volop tijd voor ontspanning en gezelligheid. Te beginnen met een feestje! 



\section{Over de auteur}

Gerdienke Prange werd op 18 juni 1981 geboren in Aalten. Na het afronden van het Voortgezet Wetenschappelijk Onderwijs aan het Christelijk College Schaersvoorde in Aalten in 1999, is ze aan de Universiteit Maastricht de studie Gezondheidswetenschappen gaan volgen. Haar stage deed ze bij Roessingh Research \& Development in Enschede, met als onderwerp het karakteriseren van loop-gerelateerde aspecten op het gebied van spieractivatie en biomechanica van patiënten met multipele sclerose. Ze studeerde in 2003 cum laude af voor de afstudeerrichting Bewegingswetenschappen. Na haar afstuderen is ze gestart met haar promotieonderzoek bij Roessingh Research \& Development, wat zich richtte op de mogelijkheden om robotica in te zetten voor de revalidatie van de armfunctie na een beroerte. Dit proefschrift is hier het resultaat van. Haar onderzoeksinteresses liggen met name op het gebied van neuromusculaire, biomechanische en klinische evaluatie van (technologische) interventies voor de revalidatie van mensen met neurologische aandoeningen. 



\section{Publications}

\section{Peer-reviewed journal articles}

Prange GB, Jannink MJA, Groothuis CGM, Hermens HJ, IJzerman MJ. A systematic review of the effect of robot-aided therapy on recovery of the hemiparetic arm after stroke. J Rehabil Res Dev 2006;43(2):171-184

Jannink MJA, Prange GB, Buurke JH, Stienen AHA, Van Asseldonk EHF, Van der Kooij $\mathrm{H}$. CVA-revalidatie van beperkte arm- en handfunctie: Virtual Reality en Robotica? Ned Tijdschr Fysiother 2008;118(4):86-94

Prange GB, Kallenberg LAC, Jannink MJA, et al. Influence of gravity compensation on muscle activity during reach and retrieval in healthy elderly. J Electromyogr Kinesiol 2009; 19(2):e40-e49

Prange GB, Jannink MJA, Stienen AHA, Van der Kooij H, IJzerman MJ, Hermens HJ. Influence of gravity compensation on muscle activation patterns during different temporal phases of arm movements of stroke patients. Neurorehab Neural Rep 2009;23(5):478-485

Prange GB, Jannink MJA, Stienen AHA, Van der Kooij H, IJzerman MJ, Hermens HJ. An explorative, cross-sectional study into abnormal muscle synergies during functional reach in chronic stroke patients. submitted 2009

Prange GB, Krabben T, Renzenbrink GJ, IJzerman MJ, Hermens HJ, Jannink MJA. Changes in muscle activation after reach training with gravity compensation in chronic stroke patients. submitted 2009

Stienen AHA, Hekman EEG, Prange GB, Jannink MJA, Aalsma AMM, Van der Helm FCT, Van der Kooij H. Dampace: design of an exoskeleton for force-coordination training in upperextremity rehabilitation. ASME J Med Dev 2009 (in press)

Stienen AHA, Hekman EEG, Prange GB, Jannink MJA, Van der Helm FCT, Van der Kooij $\mathrm{H}$. Freebal: design of a minimal weight-support system for upper-extremity rehabilitation. submitted 2009 


\section{Contributions to international conferences}

Prange GB, Jannink MJA, Kallenberg LAC, IJzerman MJ, and Hermens $\mathrm{HJ}$. Muscle activation patterns during independent and gravity-compensated reach and retrieval movements in elderly persons. In: Proceedings of the 16th Congress of the Internation Society of Electrophysiology and Kinesiology; June 28 - July 1, 2006, Turin, Italy: 138

Stienen AHA, Van der Helm FCT, Prange GB, Jannink MJA, and Van der Kooij $H$. Effects of gravity compensation on the range-of-motion of the upper extremities in robotic rehabilitation after stroke. In: Proc Inter Shoulder Group; October 9-10, 2006, Chicago (IL), USA

Jannink MJA, Prange GB, Stienen AHA, Van der Kooij $H$, Kruitbosch JM, IJzerman $\mathrm{MJ}$, and Hermens $\mathrm{HJ}$. Reduction of muscle activity during repeated reach and retrieval with gravity compensation in stroke patients. In: Proceedings of the 10th International Conference on Rehabilitation Robotics (ICORR); June 13-15, 2007, Noordwijk aan Zee, the Netherlands: 472-476

Prange GB, Stienen AHA, Jannink MJA, Van der Kooij $H$, IJzerman MJ, and Hermens $\mathrm{HJ}$. Increased range of motion and decreased muscle activity during maximal reach with gravity compensation in stroke patients. In: Proceedings of the 10th International Conference on Rehabilitation Robotics (ICORR); June 13-15, 2007, Noordwijk aan Zee, the Netherlands: 467-471

Stienen AHA, Hekman EEG, Van der Helm FCT, Prange GB, Jannink MJA, Aalsma AMM, and Van der Kooij H. Dampace: dynamic force-coordination trainer for the upper extremities. In: Proceedings of the 10th International Conference on Rehabilitation Robotics (ICORR); June 13-15, 2007, Noordwijk aan Zee, the Netherlands: $820-826$

Stienen AHA, Hekman EEG, Van der Helm FCT, Prange GB, Jannink MJA, Aalsma AMM, and Van der Kooij $\mathrm{H}$. Freebal: dedicated gravity compensation for het upper extremities. In: Proceedings of the 10th International Conference on Rehabilitation Robotics (ICORR); June 13-15, 2007, Noordwijk aan Zee, the Netherlands: 804808 
Prange GB, Krabben T, Molier BI, Van der Kooij $\mathrm{H}$, and Jannink MJA. A low-tech virtual reality application for training of upper extremity motor function in neurorehabilitation. In: Proceedings of Virtual Rehabilitation 2008; 25-27 August 2008; Vancouver, Canada.

Prange GB, Krabben T, De Boer J, Renzenbrink GJ, Hermens HJ, and Jannink MJA. Preliminary findings of gravity compensation training in combination with a rehabilitation game in chronic stroke patients. At: International NeuroRehabilitation Symposium (INRS); February 12-13, 2009, Zürich, Switzerland

Prange GB, Jannink MJA, Stienen AHA, Van der Kooij $H$, IJzerman MJ, and Hermens $\mathrm{HJ}$. Gravity compensation during repetitive and maximal reach after stroke. At: Technically Assisted Rehabilitation (TAR) conference; March 18-19, 2009, Berlin, Germany

Prange GB, Krabben T, Renzenbrink GJ, de Boer J, Hermens HJ, and Jannink MJA. An explorative study into changes in reach performance after gravity compensation training in chronic stroke patients. In: Proceedings of the $11^{\text {th }}$ International Conference on Rehabilitation Robotics (ICORR); June 23-26, 2009, Kyoto, Japan

Van der Kooij H, Prange GB, Krabben T, Renzenbrink GJ, De Boer J, Hermens HJ, and Jannink MJA. Preliminary results of training with gravity compensation of the arm in chronic stroke survivors. At: IEEE Engineering in Medicine and Biology Society Conference (EMBC); September 2-6, 2009, Minneapolis (MN), USA 



\section{Progress Range}

The following publications have also been published in the Progress Range by Roessingh Research and Development, Enschede, the Netherlands. Copies can be ordered, when available, via info@rrd.nl.

1. Pot JWGA, Boer H, van Harten WH, Hermens HJ, Seydel ER. Comprehensive Need-Assessment. Ontwikkeling van een meetinstrument voor zorgbehoeften en kwaliteitsbeoordeling door patiënten. Roessingh Research and Development, the Netherlands, September 1994, ISBN 90-25452-01-2

2. Van Leerdam NGA, Hermens $\mathrm{HJ}$. Revalidatietechnologie in Euregio. Roessingh Research and Development, the Netherlands, July 1995, ISBN 90-75452-02-0

3. Duda L, van Noort LO, Röseler S, Greitemann BOL, van Harten WH, Klazinga NS. Rehabilitation in Germany and the Netherlands, A comparison of two rehabilitation-systems. Roessingh Research and Development, the Netherlands, August 1995, ISBN 90-75452-03-9

4. Hermens HJ, Nene AV, Zilvold G. Electrophysiological Kinesiology. Proceedings of the 11th congress of the International Society of Electrophysiologie and Kinesiology in Enschede, the Netherlands 1996, Roessingh Research and Development, the Netherlands, October 1996, ISBN 90-75452-04-7

5. Van Harten WH. Bouwen aan een kwaliteitssysteem in de revalidatiezorg. Een poging tot constructieve technology assessment van een kwaliteitssysteem in een gezondheidszorginstelling. Roessingh Research and Development, the Netherlands, December 1997, ISBN 90-75452-07-1

6. Baardman G, IJzerman MJ. Design and evaluation of a hybrid orthosis for people with paraplegia. Roessingh Research and Development, the Netherlands, November 1997, ISBN 90-75452-08-X

7. Hutten MMR. Lumbar Dynamometry: A useful method for assessment of patients with chronic low back pain? Roessingh Research and Development, the Netherlands, November 1999, ISBN 90-75452-13-6

8. Van der Salm A, van Harten WH, Maathuis CGB. Ketenkwaliteit Cerebrale Parese Zorg. Een beschrijving van de cerebrale parese zorg en mogelijke verbeteringen hierin. Roessingh Research and Development, the Netherlands, April 2001, ISBN 90-75452-19-5 
9. Nederhand MJ. Muscle activation patterns in post traumatic neck pain. Roessingh Research and Development, the Netherlands, March 2003, ISBN 9075452-27-6

10. Jannink MJA. Usability of custom-made orthopaedic shoes in patients with degenerative disorders of the foot. Roessingh Research and Development, the Netherlands, September 2004, ISBN 90-75452-28-4

11. Blokhorst, M. State-dependent factors and attention in whiplash associated disorder, Roessingh Research and Development, the Netherlands, December 2005, ISBN 90-365-2111-4

12. Buurke JH. Walking after stroke co-ordination patterns \& functional recovery. Roessingh Research and Development, the Netherlands, February 2005, ISBN 90-365-2140-8

13. Van der Salm A. Spasticity reduction using electrical stimulation in the lower limb of spinal cord injury patients. Roessingh Research and Development, the Netherlands, October 2005, ISBN 90-365-2253-6

14. Snoek GJ. Patient preferences for reconstructive interventions of the upper limb in tetraplegia. Roessingh Research and Development, the Netherlands, December 2005, ISBN 90-365-2255-2

15. De Kroon J. Therapeutic electrical stimulation of the upper extremity in stroke. Roessingh Research and Development, the Netherlands, December 2005, ISBN 90-365-2269-2

16. Van Dijk H. Motor skill learning, age and augmented feedback. Roessingh Research and Development, the Netherlands, March 2006, ISBN 90-365-23029

17. Mes CAJ. Improving non-optimal results in chronic pain treatment. Roessingh Research and Development, the Netherlands, January 2007, ISBN 90-3652435-0

18. Voerman GE. Musculoskeletal neck-shoulder pain: a new ambulant myofeedback intervention approach. Roessingh Research and Development, the Netherlands, March 2007, ISBN 90-365-2460-1

19. Kallenberg LAC. Multi-channel array EMG in chronic neck-shoulder pain. Roessingh Research and Development, the Netherlands, March 2007, ISBN 90365-2459-8

20. Huis in 't Veld MHA. Work-related neck-shoulder pain: The role of cognitivebehavioural factors and remotely supervised treatment. Roessingh Research and Development, the Netherlands, December 2007, ISBN 978-90-365-2584-8 
21. Fleuren JFM. Assessment of spasticity - from EMG to patients' perception. Roessingh Research and Development, the Netherlands, October 2009, ISBN 978-90-365-2869-6

22. Reenalda J. Dynamic sitting to prevent pressure ulcers in spinal cord injured. Roessingh Research and Development, the Netherlands, October 2009, ISBN 978-90-365-2884-9 
